\title{
An Alkylidene Carbene C-H Activation Approach Toward the Enantioselective Syntheses of Spirolactams: Application to the Synthesis of (-)-Adalinine
}

\author{
Krishna Annadi and Andrew G. H. Wee* \\ Department of Chemistry and Biochemistry, University of Regina \\ Regina, Saskatchewan, S4S 0A2, Canada.
}

\section{$\underline{\text { Table of Contents }}$}

\section{Contents}

Pages

${ }^{1} \mathrm{H}$ and ${ }^{13} \mathrm{C}$ NMR spectra for compounds $(S, S)-8 \mathrm{c} ; \mathbf{9} ; \mathbf{1 0} ; \mathbf{1 1} ; \mathbf{1 2}$; $(S, S)-7 \mathbf{e} ;(S, S)-8 \mathbf{a} ;(R, R)-\mathbf{8 b} ;(S, S)-\mathbf{1 4 a} ;(R, R)-\mathbf{1 4 b} ;(S, S)-\mathbf{1 5 a} \mathbf{a}\left({ }^{1} \mathrm{H}\right.$ only); $(R, R)-\mathbf{1 5 b}\left({ }^{1} \mathrm{H}\right.$ only); $(S, S)-13 \mathbf{a} ;(R, R)-13 \mathbf{b} ;(S, S)-$

15a.thiocarbonate; $(R, R)-15 \mathbf{b}$.thiocarbonate; $(R)-13 \mathbf{c} ;(S)-13 d$;

$(S, S)-7 \mathbf{a} ;(S, S)-\mathbf{1 6} \mathbf{a}\left({ }^{1} \mathrm{H}\right.$ only); $(R, R)-7 \mathbf{b} ;(R, R)-\mathbf{1 6 b}\left({ }^{1} \mathrm{H}\right.$ only); $(S)-7 \mathbf{c}$;

$(S)-16 \mathbf{c} ;(S)-7 \mathbf{d} ;(S)-16 \mathbf{d} ; 17 \mathbf{a} ; 17 \mathbf{b} ; 17 \mathbf{d} ; 18 \mathbf{a}+19 \mathbf{a}\left({ }^{1} \mathrm{H}\right.$ only);

$18 \mathrm{~b}+19 \mathrm{~b} ; 19 \mathrm{c} ; 20 \mathrm{a} ; 21 ; 22 ; 23\left({ }^{1} \mathrm{H}\right.$ only); 20b; 24; 20c; 20d; 20e; 31

$\left({ }^{1} \mathrm{H}\right.$ only); 31.thiocarbonate; 32; 30; 33; $(S)-29 ;(S)-28 ;(S)-35 ;( \pm)-$ 36; ( \pm )-37; 38 $+35 ; 39 ; 41 ; 42 ; 43$; Debenzylated 43; 2 .

S2-S110

HPLC characterization data for compounds 29 and 2.

S111-S112 


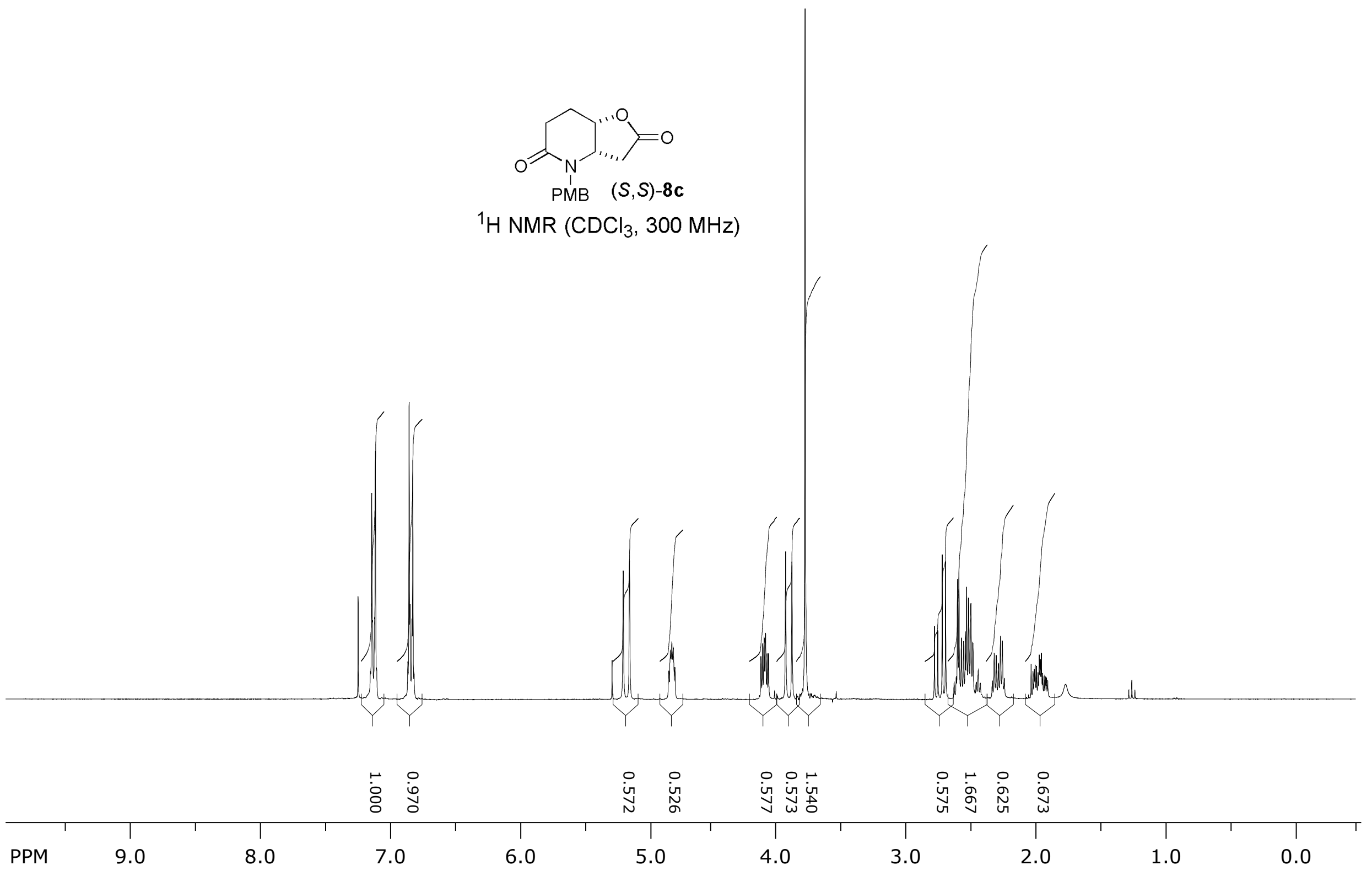

S2 


\begin{tabular}{|c|c|c|c|c|}
\hline 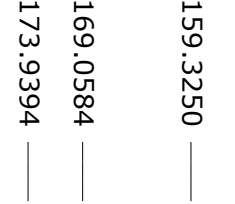 & 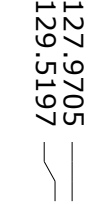 & $\begin{array}{l}\vec{b} \\
\stackrel{+}{\omega} \\
\stackrel{0}{\infty} \\
\infty\end{array}$ & 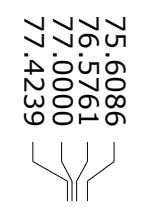 & 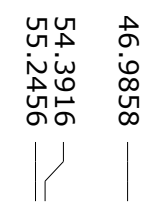 \\
\hline
\end{tabular}

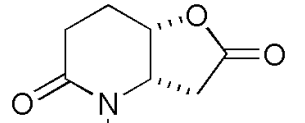

PMB $(S, S)-8 \mathrm{c}$

${ }^{13} \mathrm{C} \mathrm{NMR}\left(\mathrm{CDCl}_{3}, 75 \mathrm{MHz}\right)$

$19 m$ m.

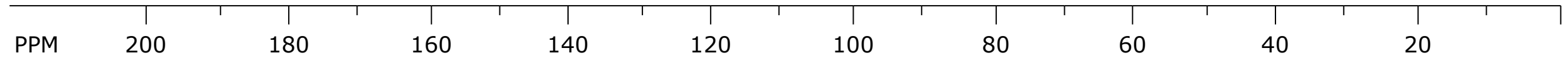




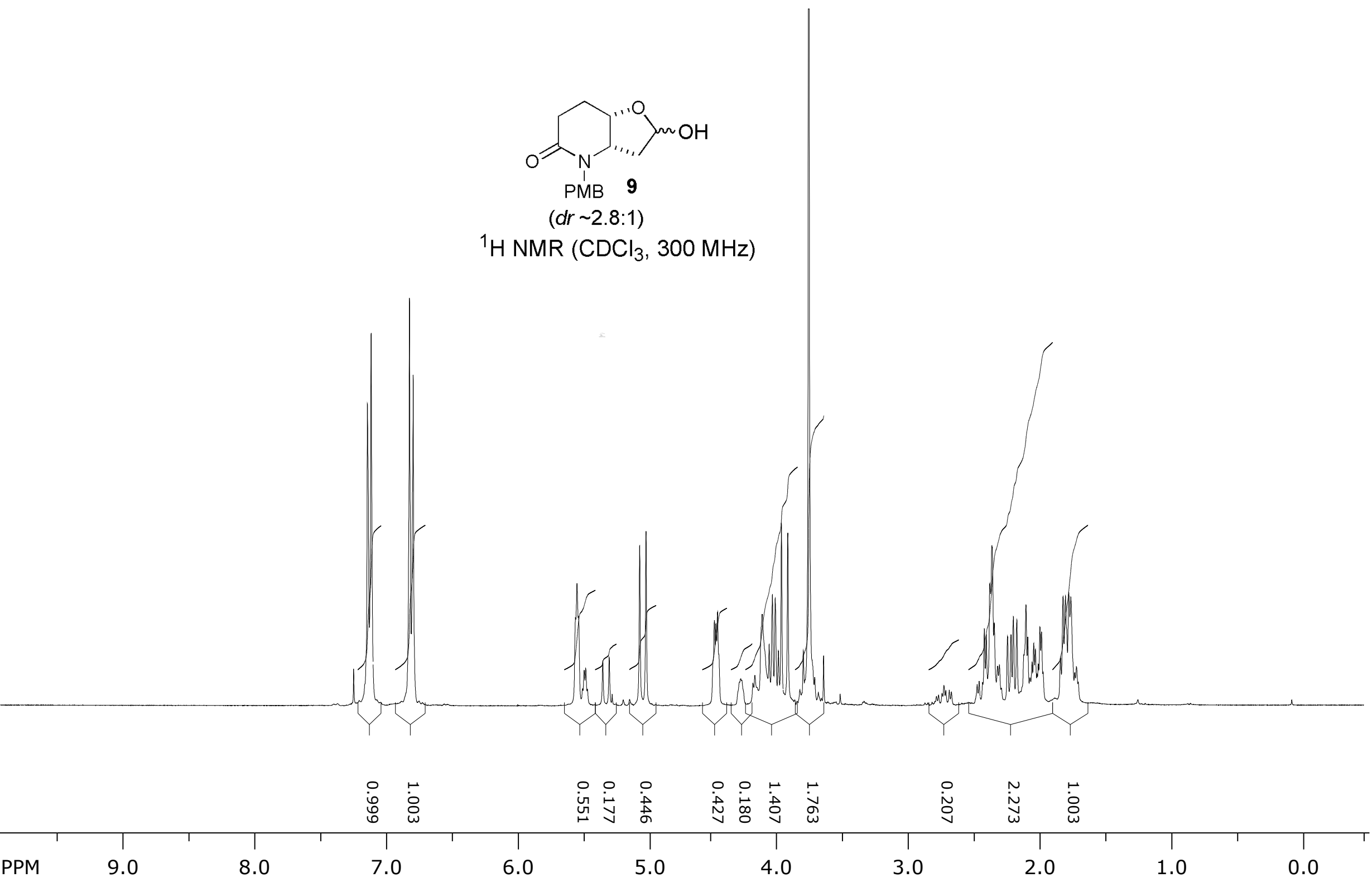




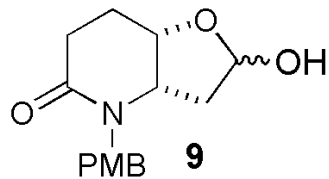

(dr 2 8:1)

${ }^{13} \mathrm{C} \mathrm{NMR}\left(\mathrm{CDCl}_{3}, 75 \mathrm{MHz}\right)$

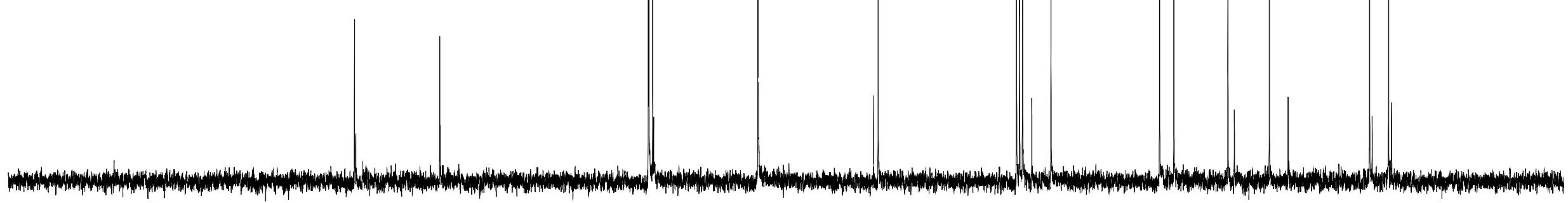




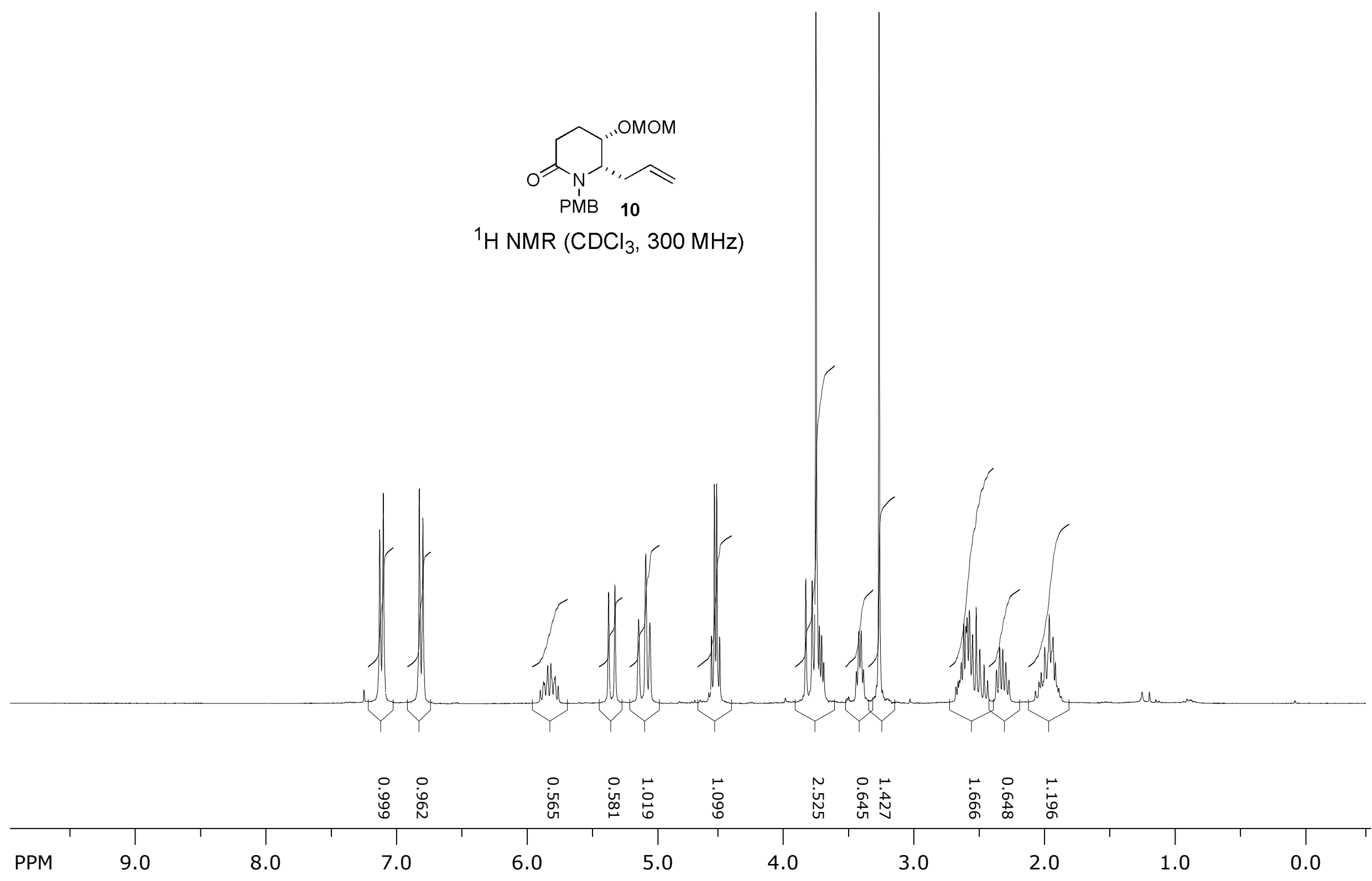




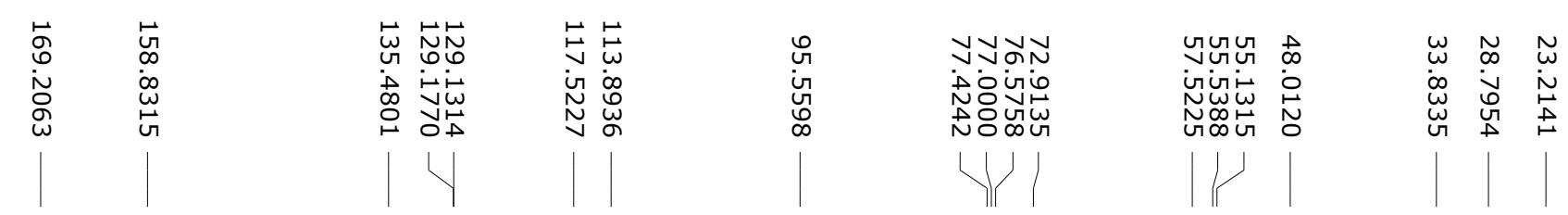

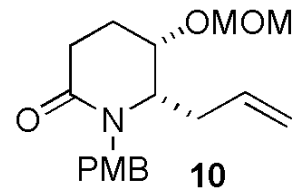

${ }^{13} \mathrm{C} \mathrm{NMR}\left(\mathrm{CDCl}_{3}, 75 \mathrm{MHz}\right)$

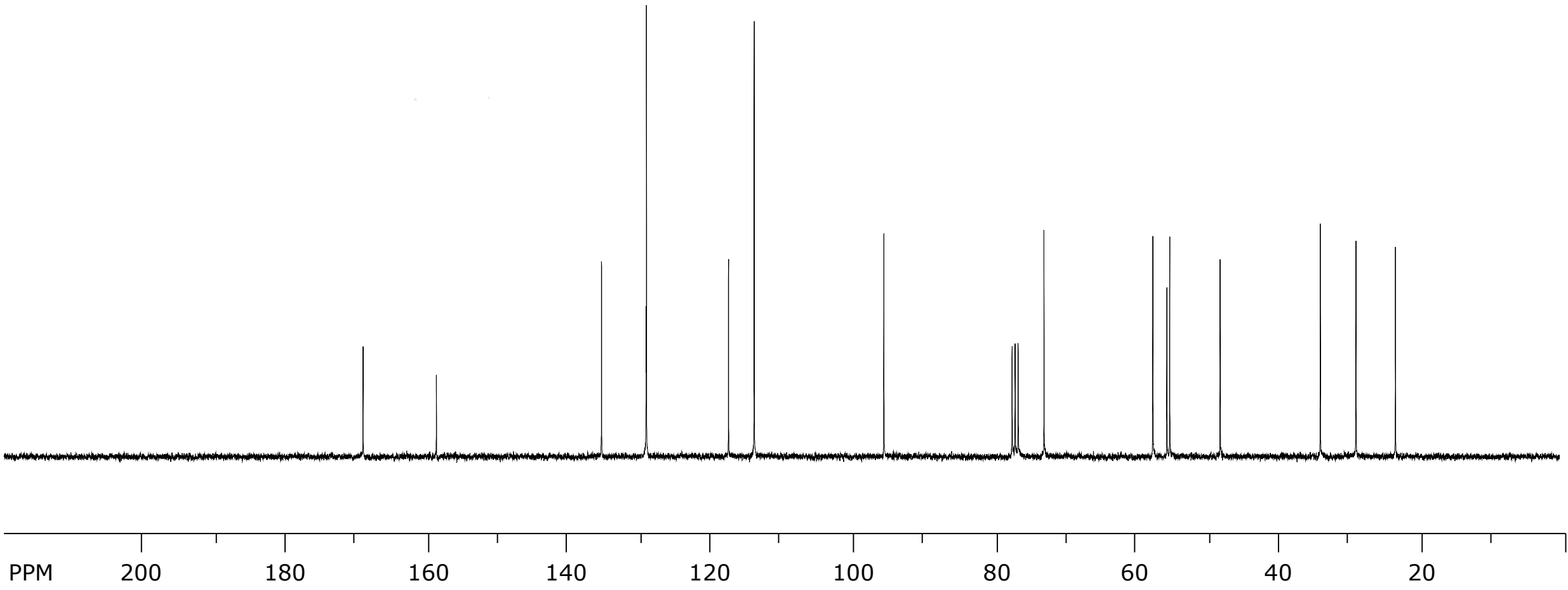




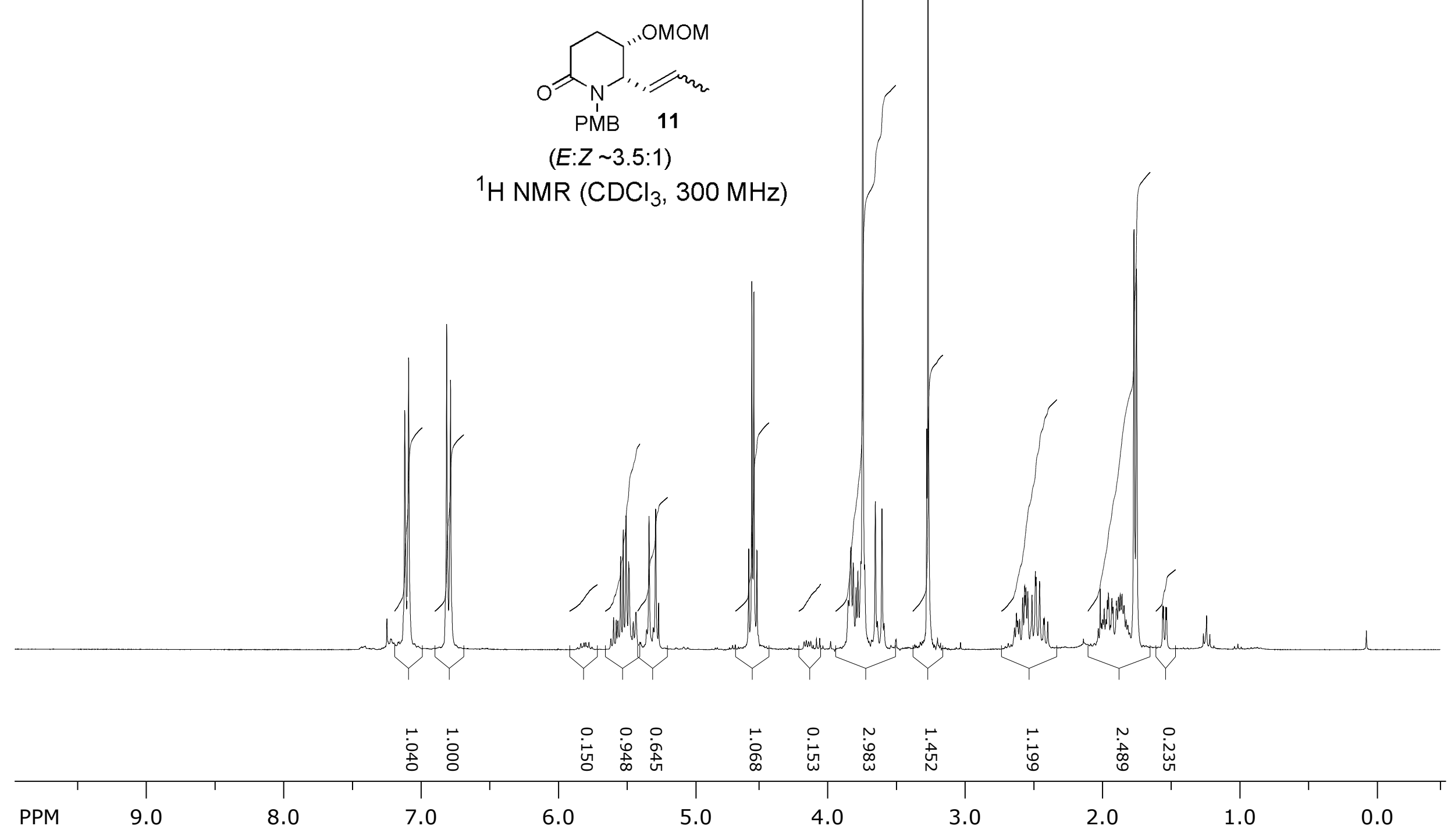



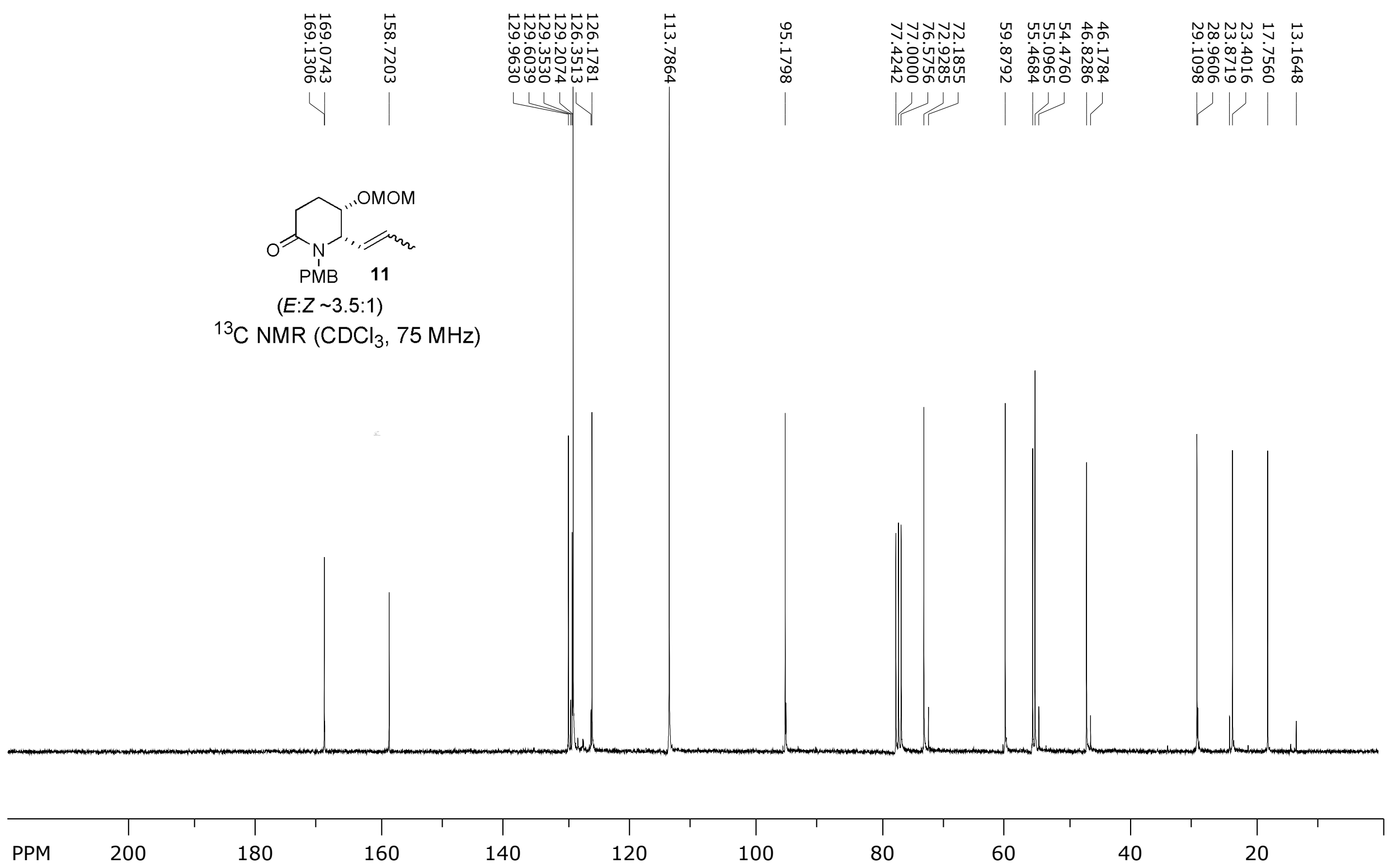


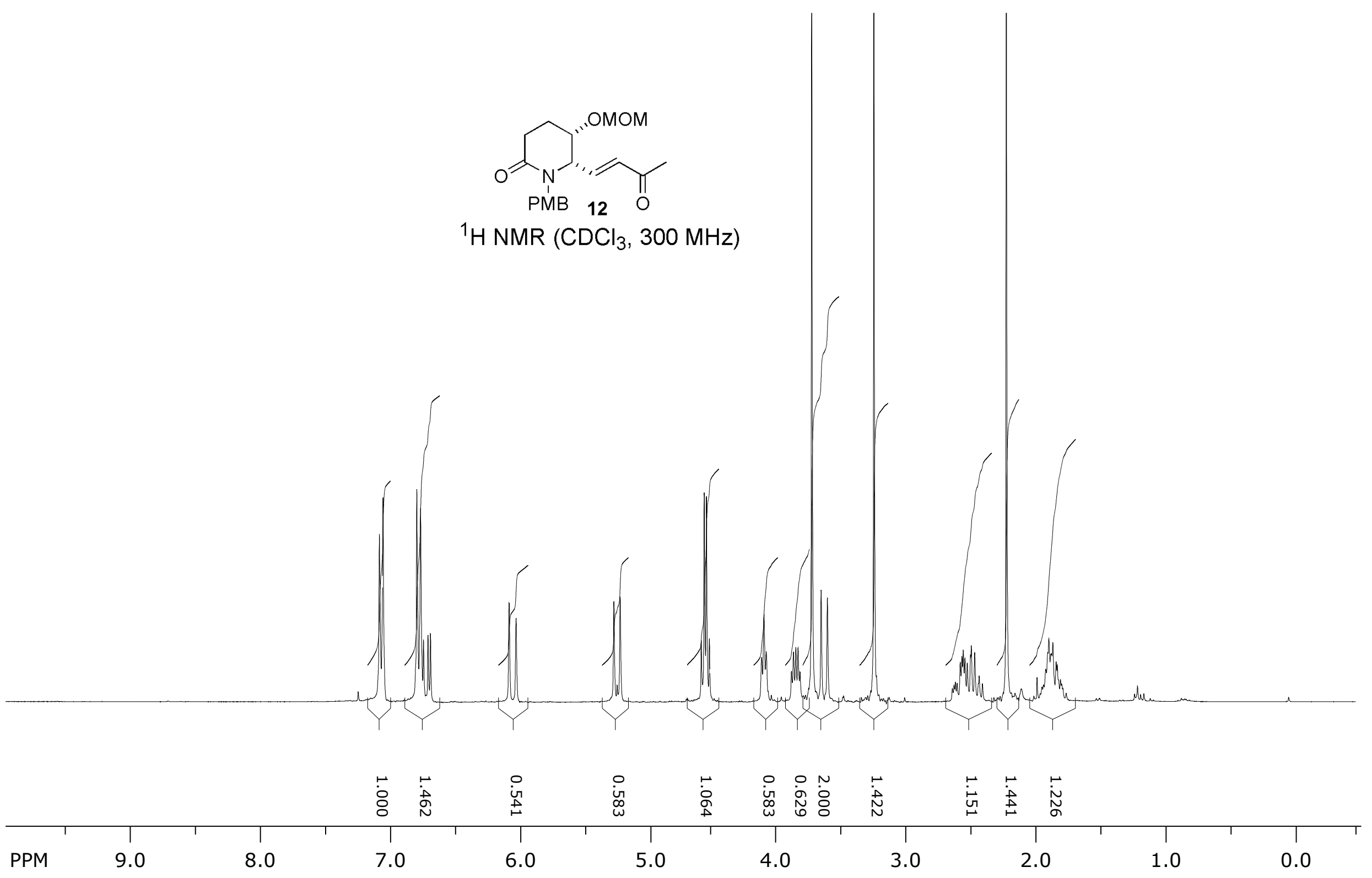

S10 


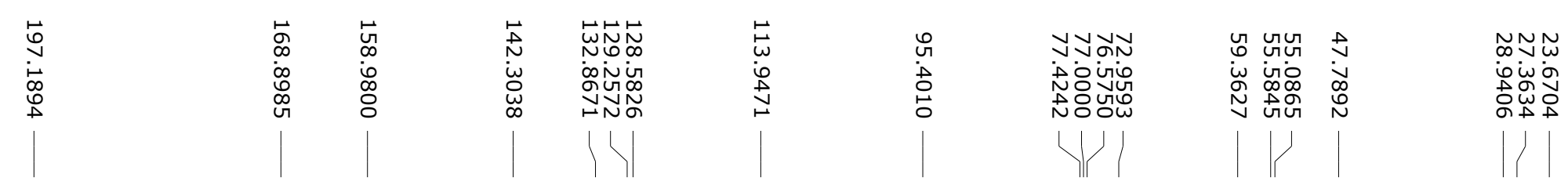

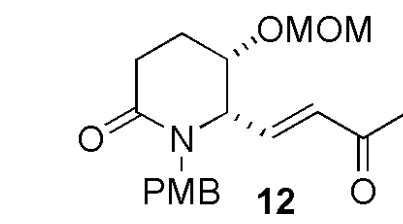

${ }^{13} \mathrm{C} \mathrm{NMR}\left(\mathrm{CDCl}_{3}, 75 \mathrm{MHz}\right)$

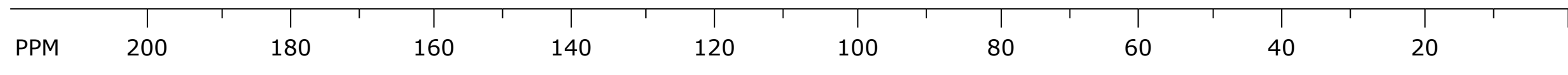




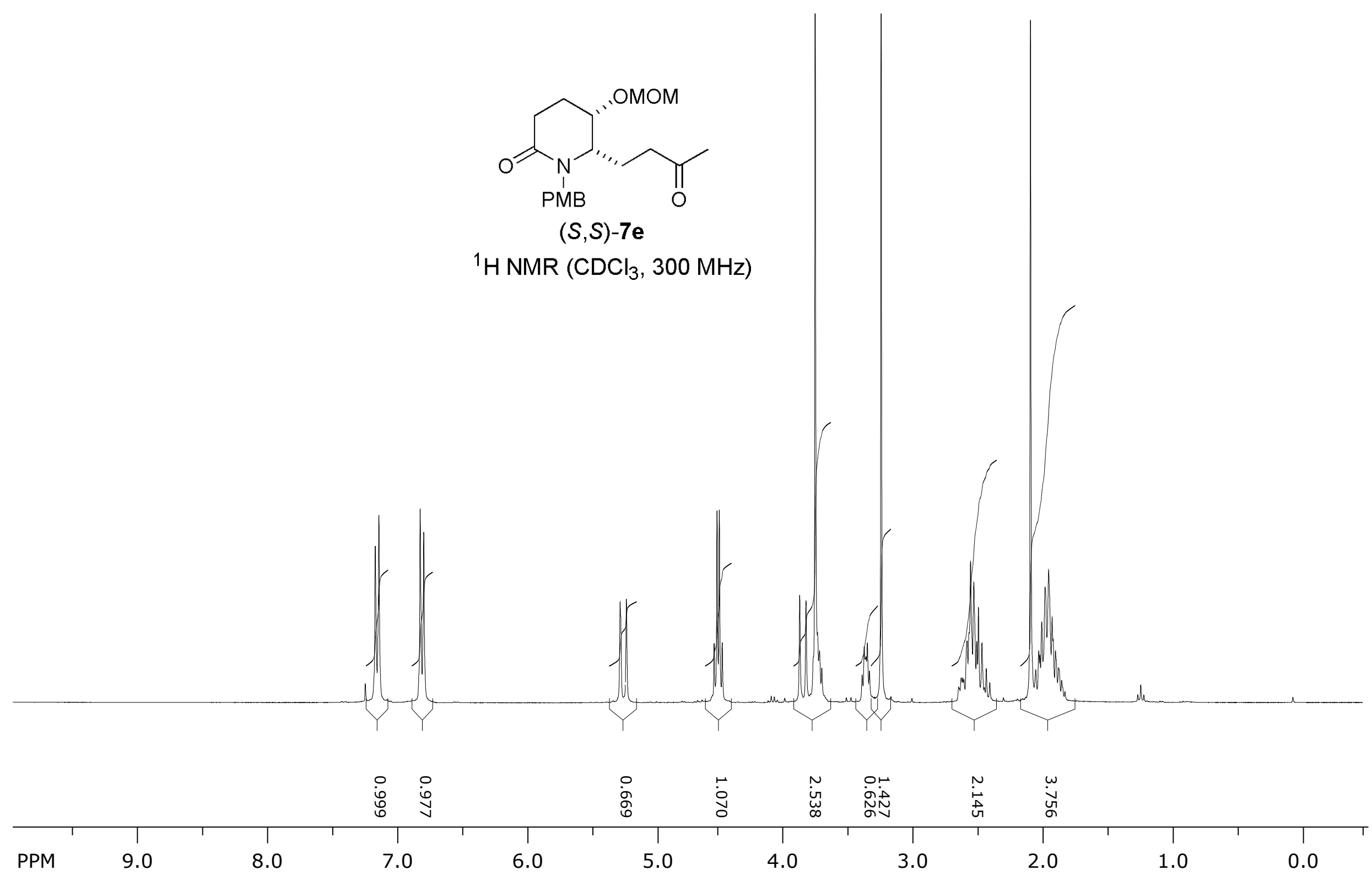




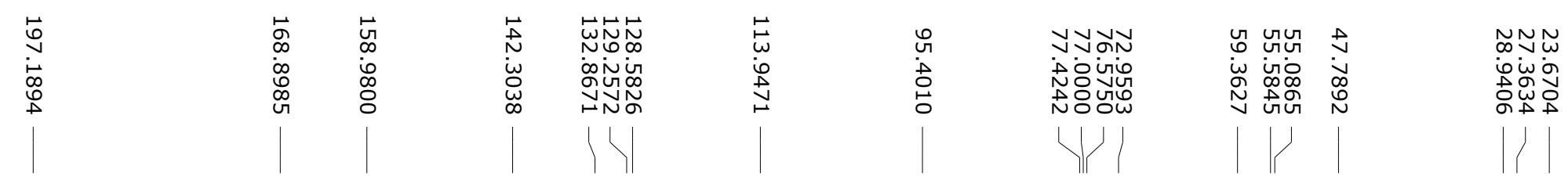

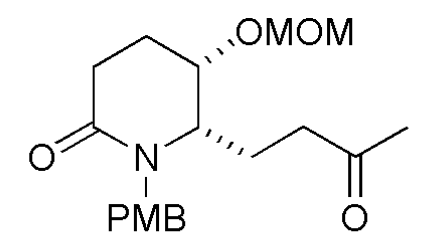

$(S, S)-7 e$

${ }^{13} \mathrm{C} \mathrm{NMR}\left(\mathrm{CDCl}_{3}, 75 \mathrm{MHz}\right)$

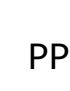




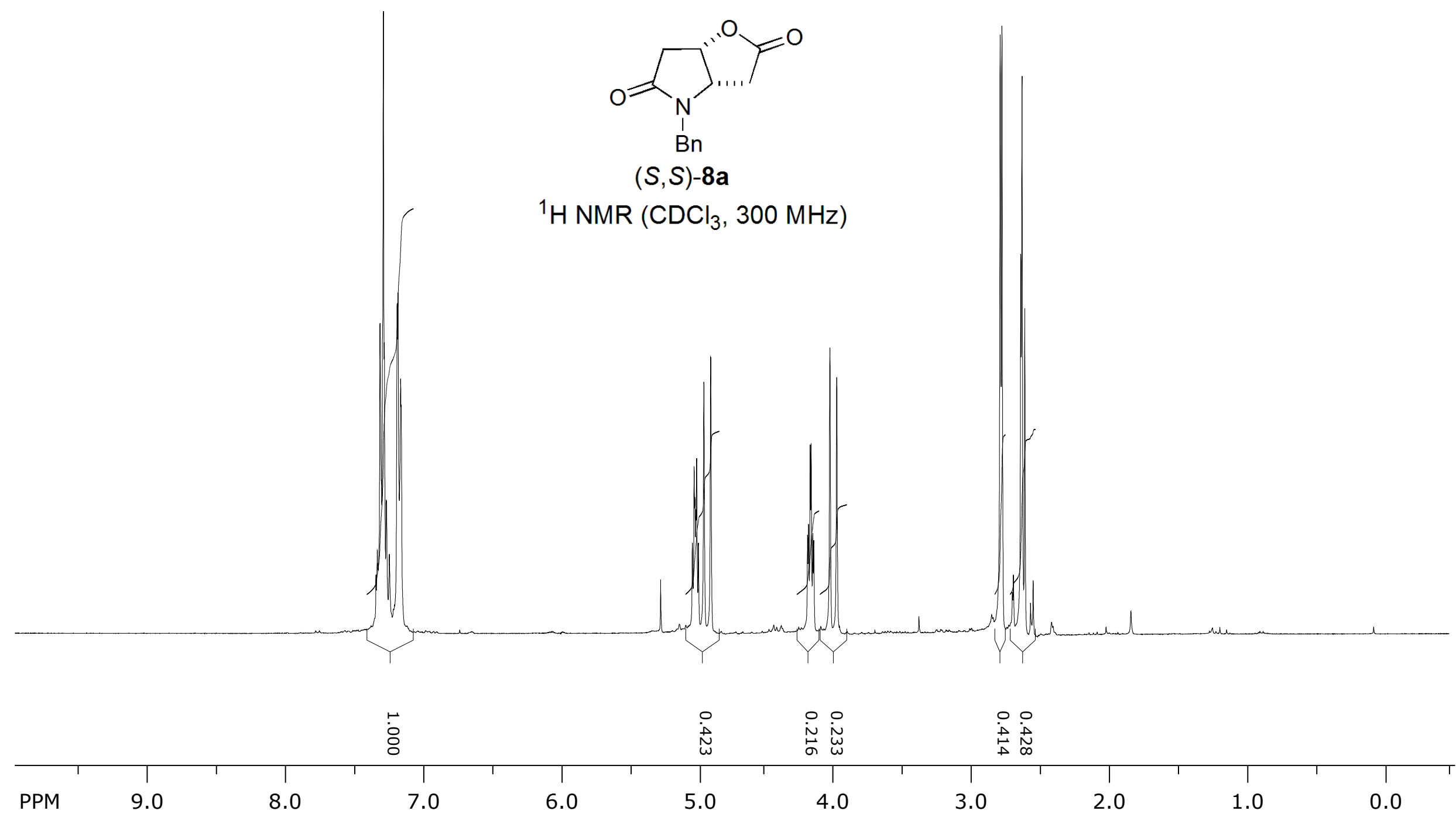




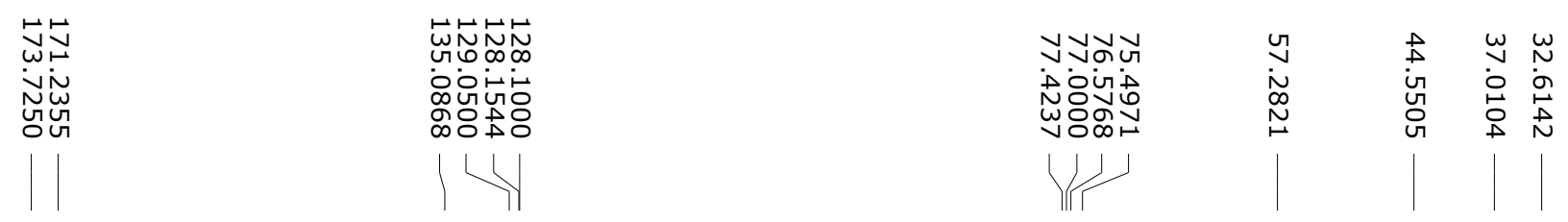

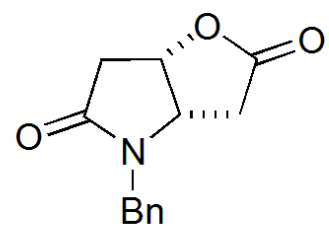

(S,S)-8a

${ }^{13} \mathrm{C} \mathrm{NMR}\left(\mathrm{CDCl}_{3}, 75 \mathrm{MHz}\right)$
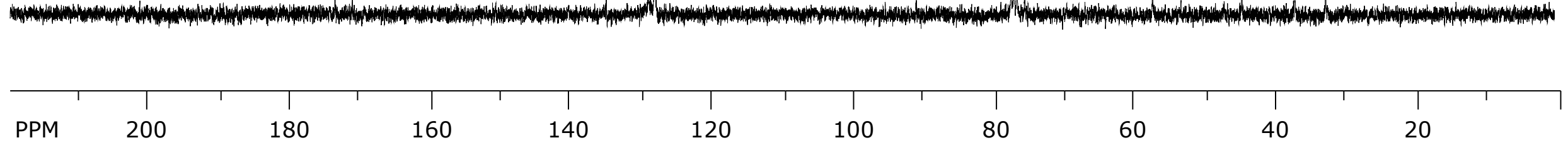


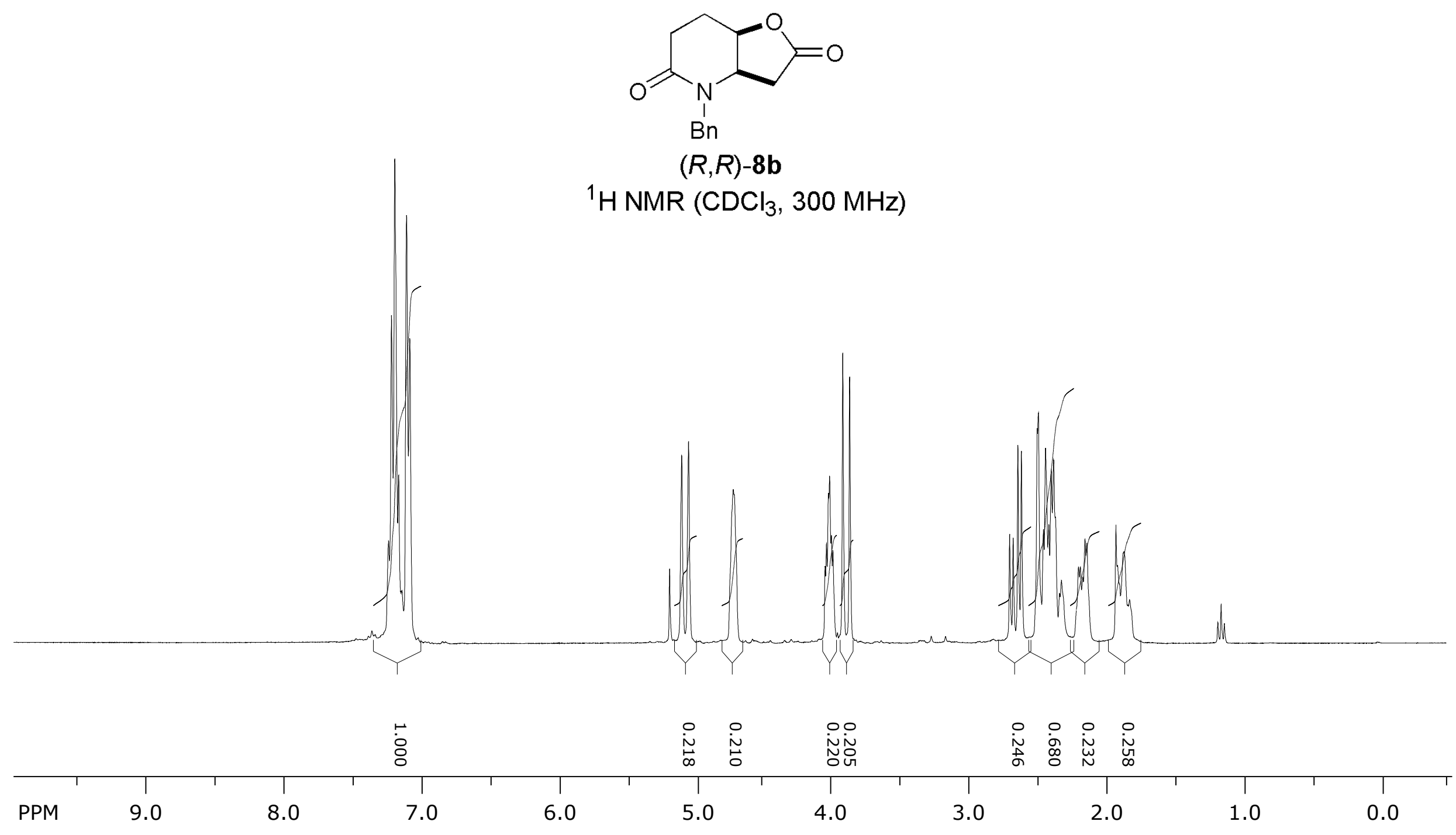




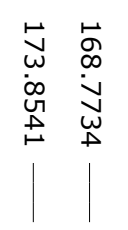

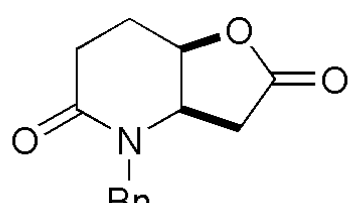

Bn

$(R, R)-\mathbf{8 b}$

${ }^{13} \mathrm{C} \mathrm{NMR}\left(\mathrm{CDCl}_{3}, 75 \mathrm{MHz}\right)$

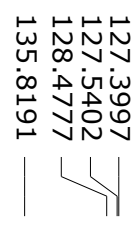

ำงับ

ํํㅇำ

Uf

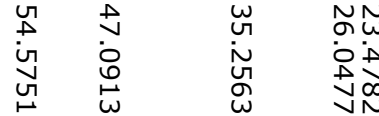

\section{$\mid$}




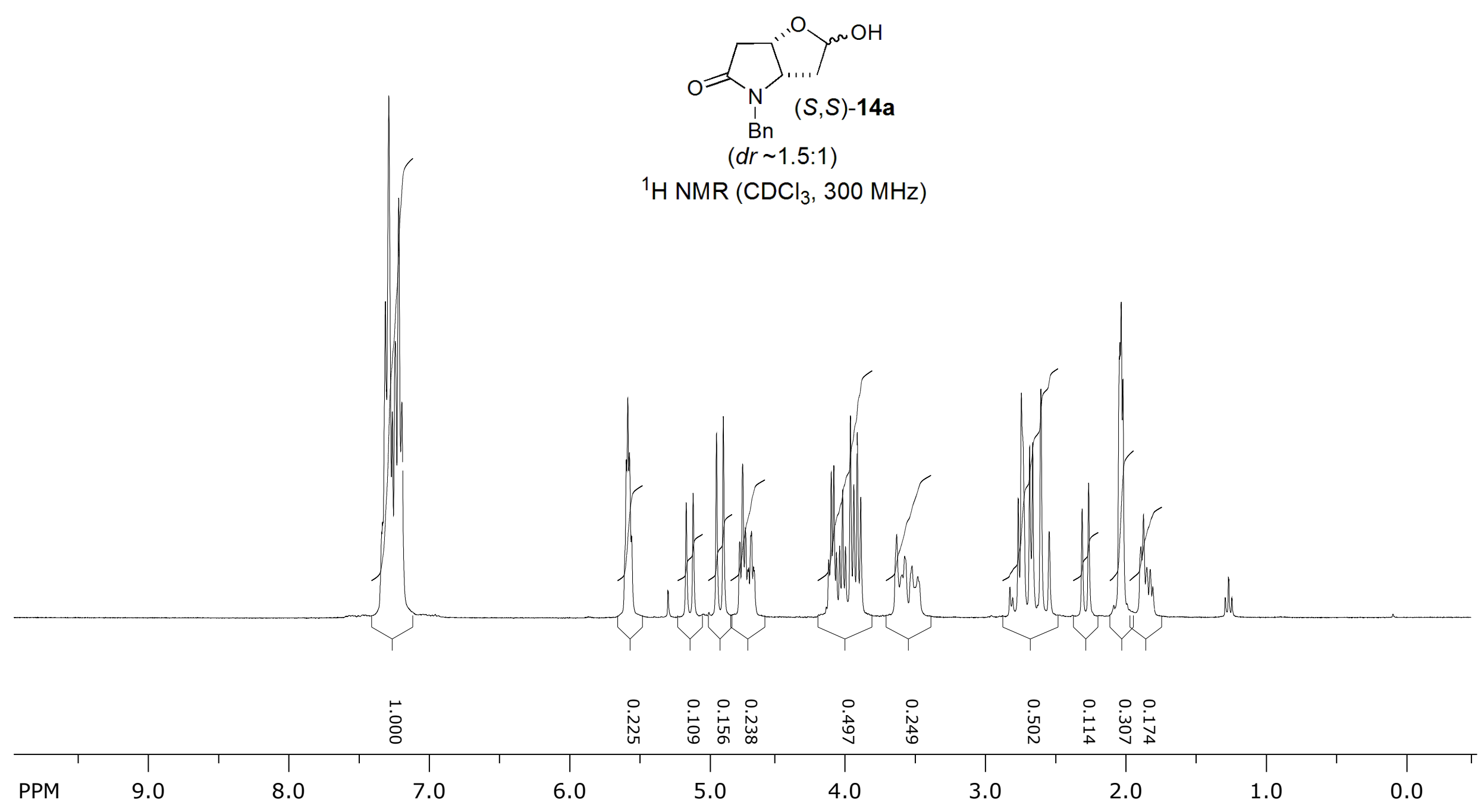



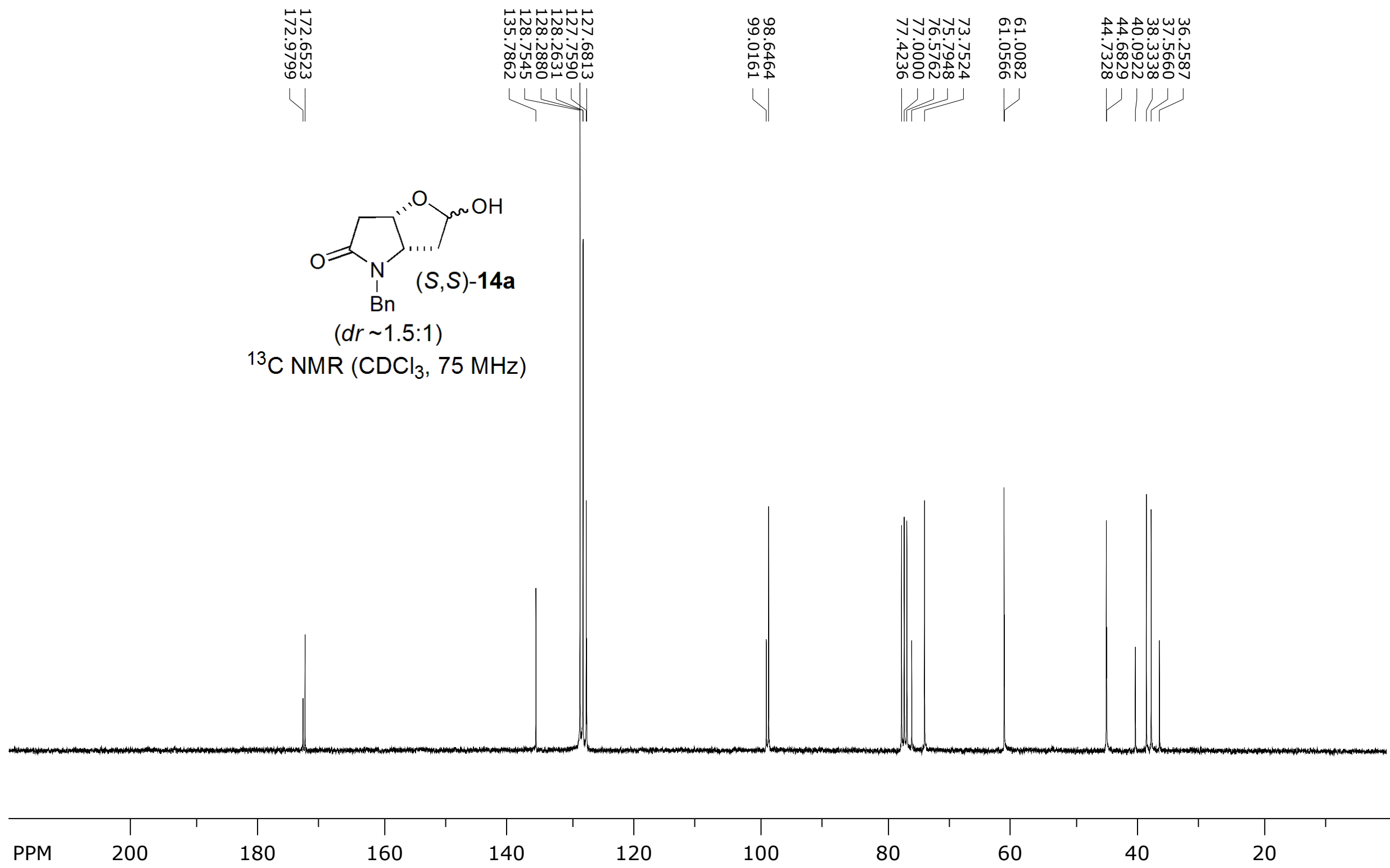


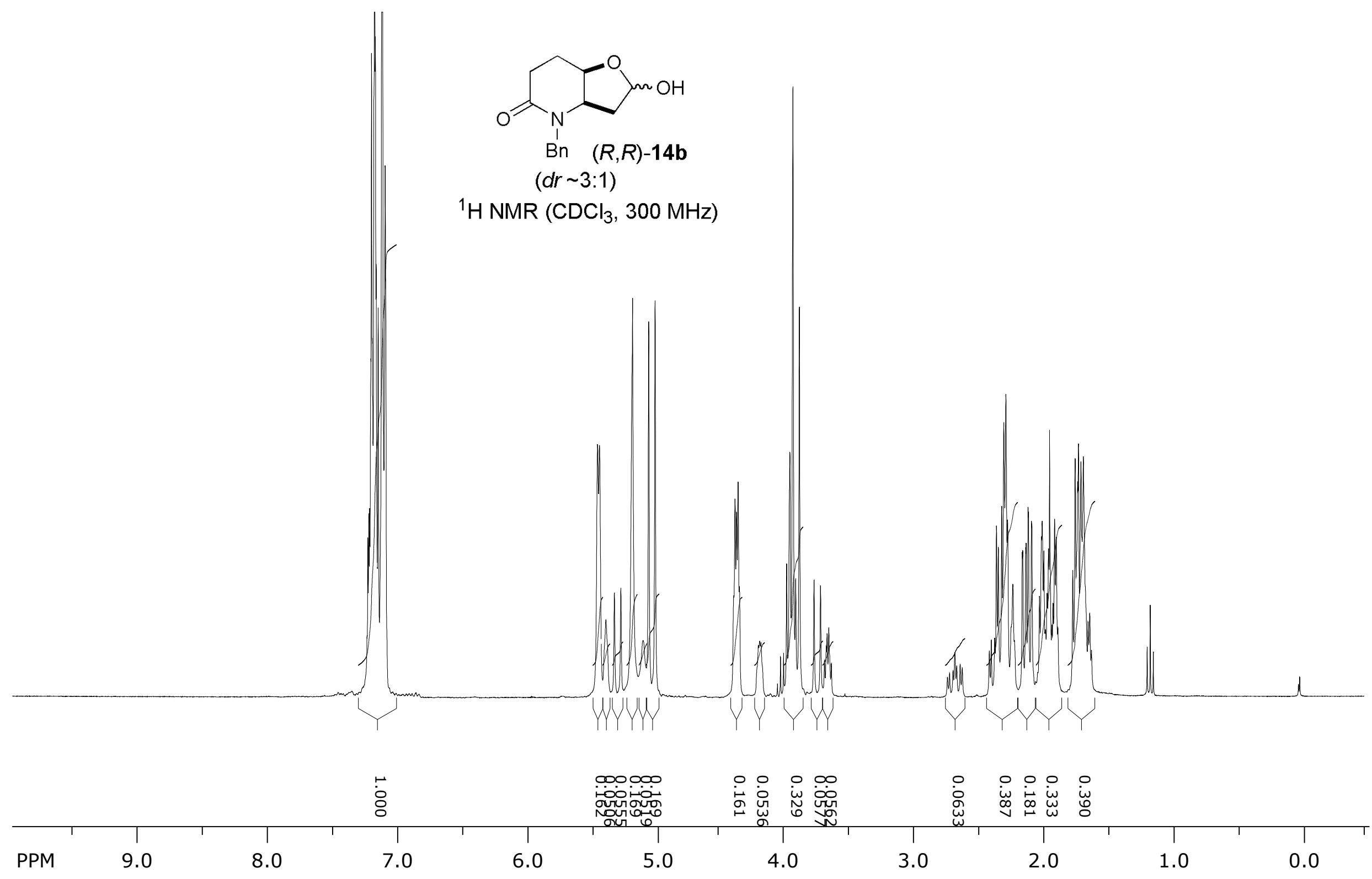




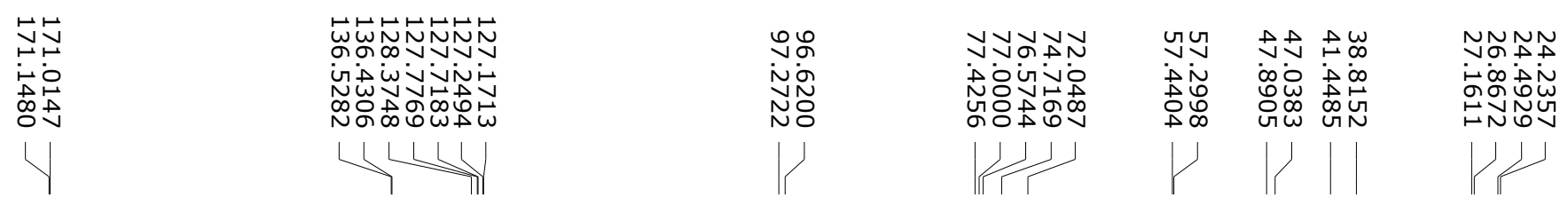

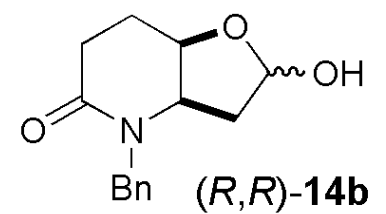

(dr 3:1)

${ }^{13} \mathrm{C} \mathrm{NMR}\left(\mathrm{CDCl}_{3}, 75 \mathrm{MHz}\right)$ 


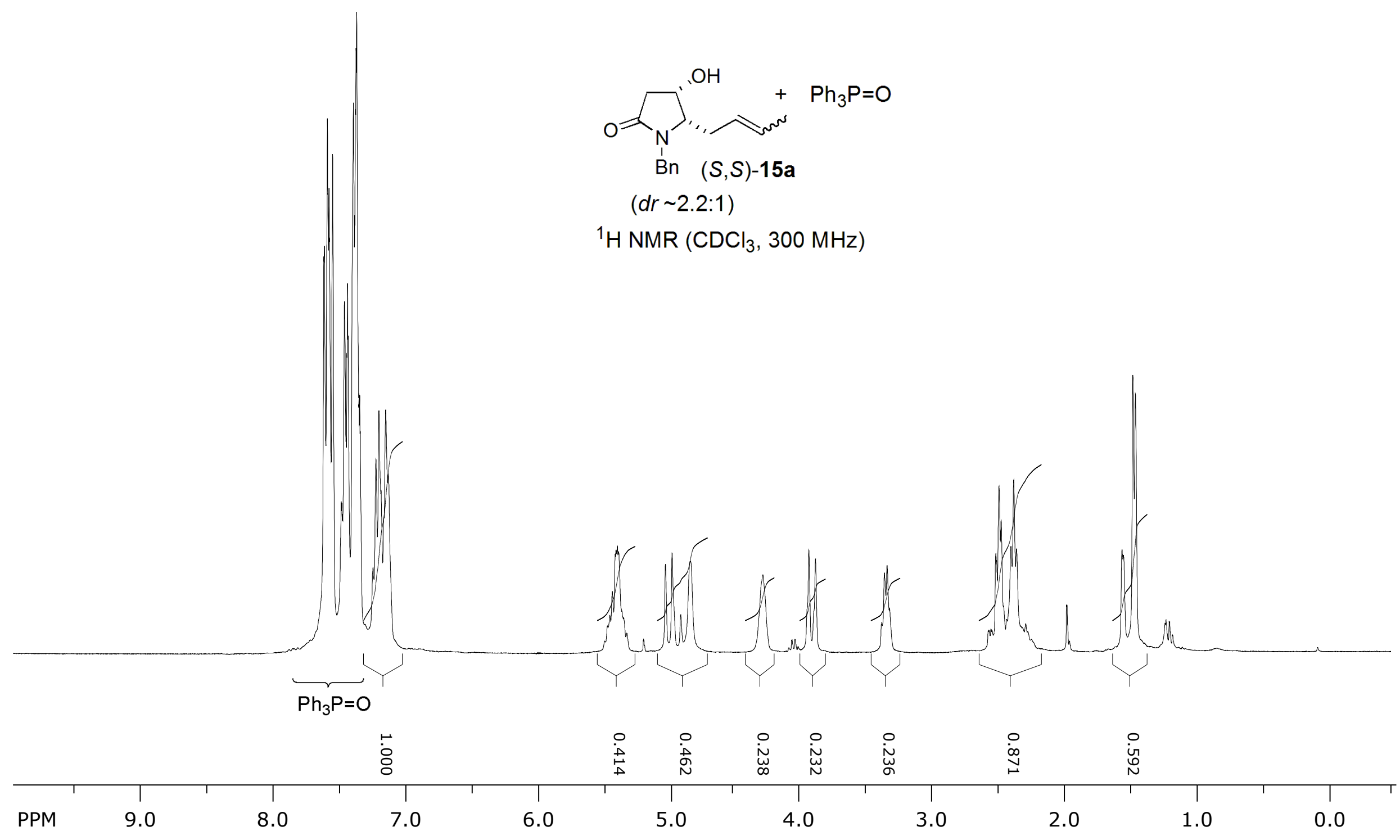




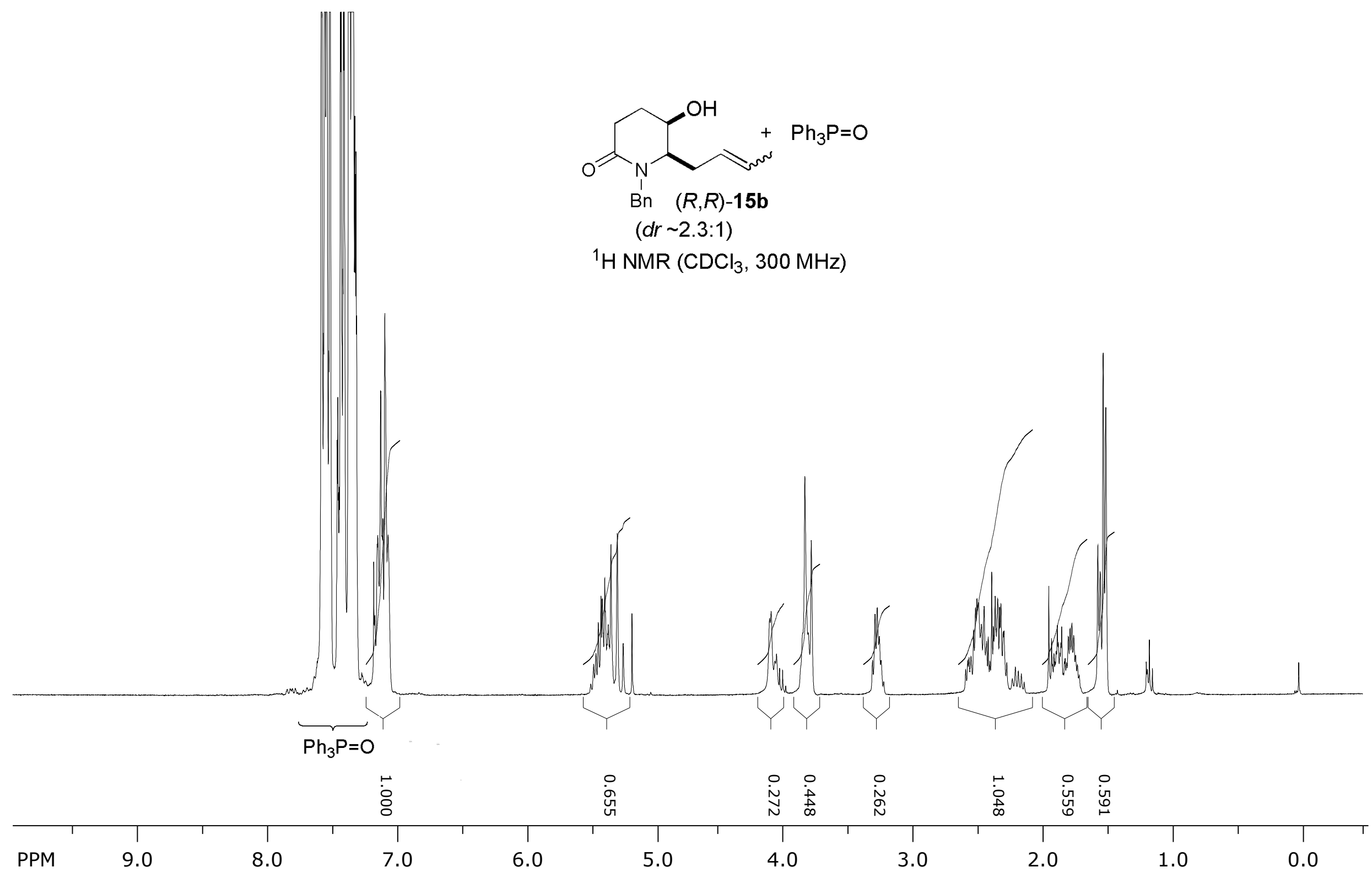




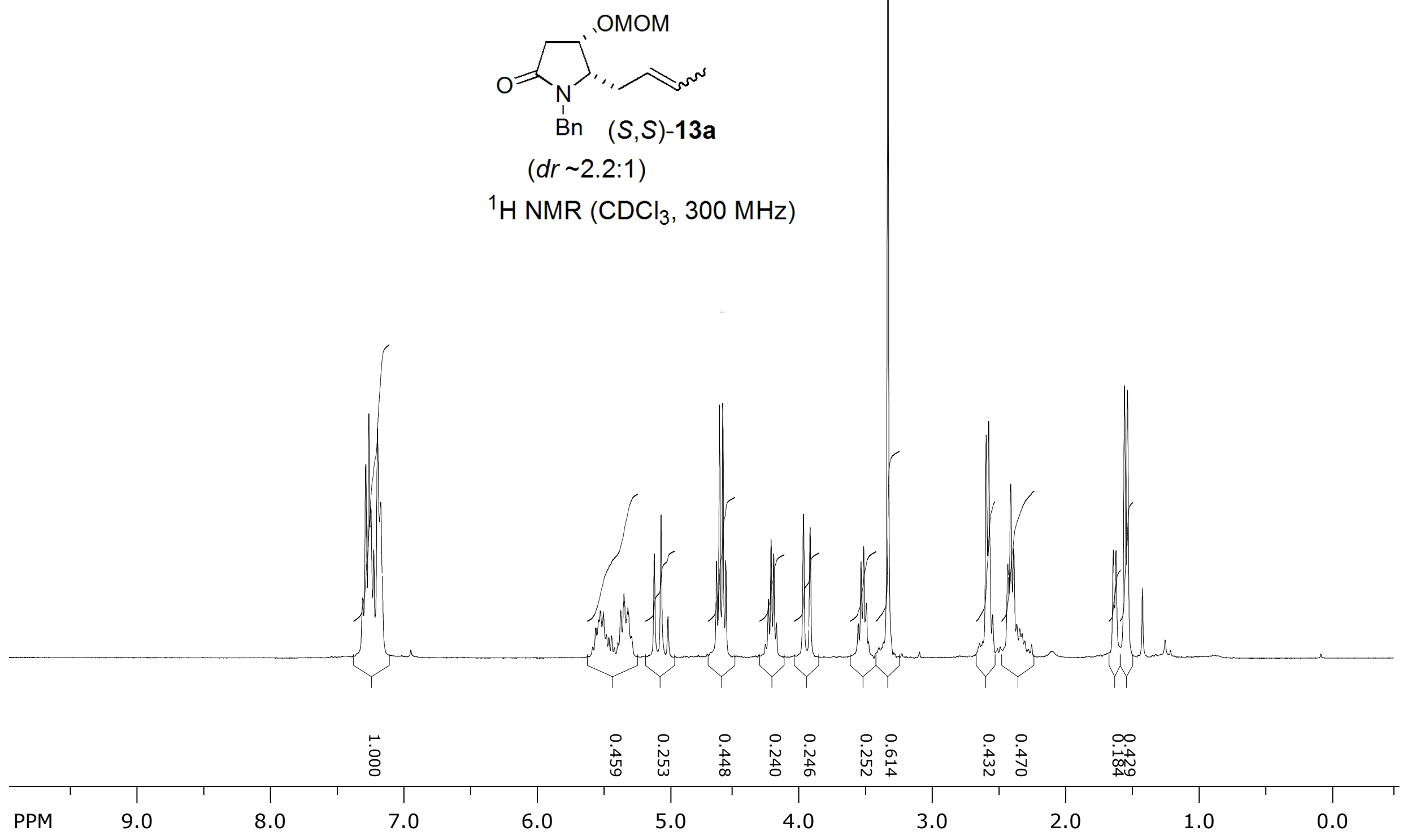




\begin{tabular}{|c|c|c|c|c|c|}
\hline 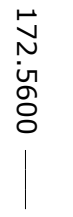 & 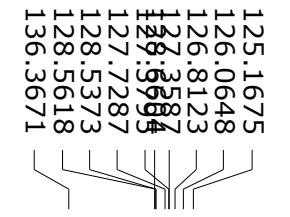 & $\begin{array}{l}0 \\
w \\
\dot{w} \\
\text { जे }\end{array}$ & 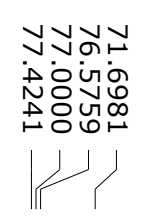 & 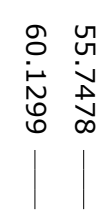 & 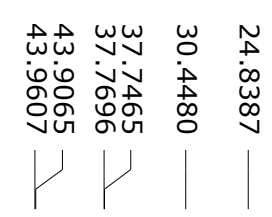 \\
\hline
\end{tabular}

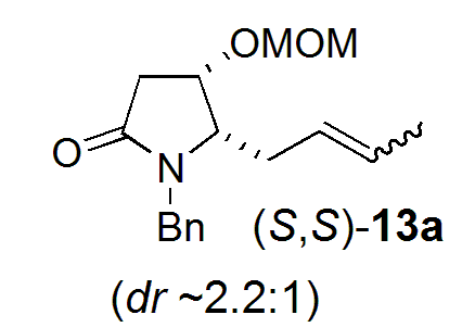

${ }^{13} \mathrm{C} \mathrm{NMR}\left(\mathrm{CDCl}_{3}, 75 \mathrm{MHz}\right)$

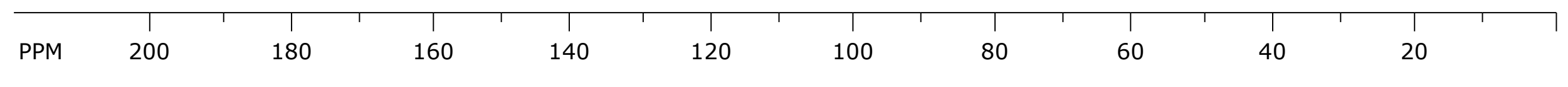




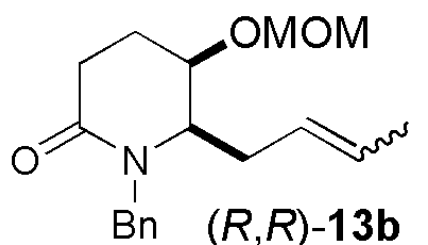

(dr 2.3:1)

${ }^{1} \mathrm{H} \mathrm{NMR}\left(\mathrm{CDCl}_{3}, 300 \mathrm{MHz}\right)$

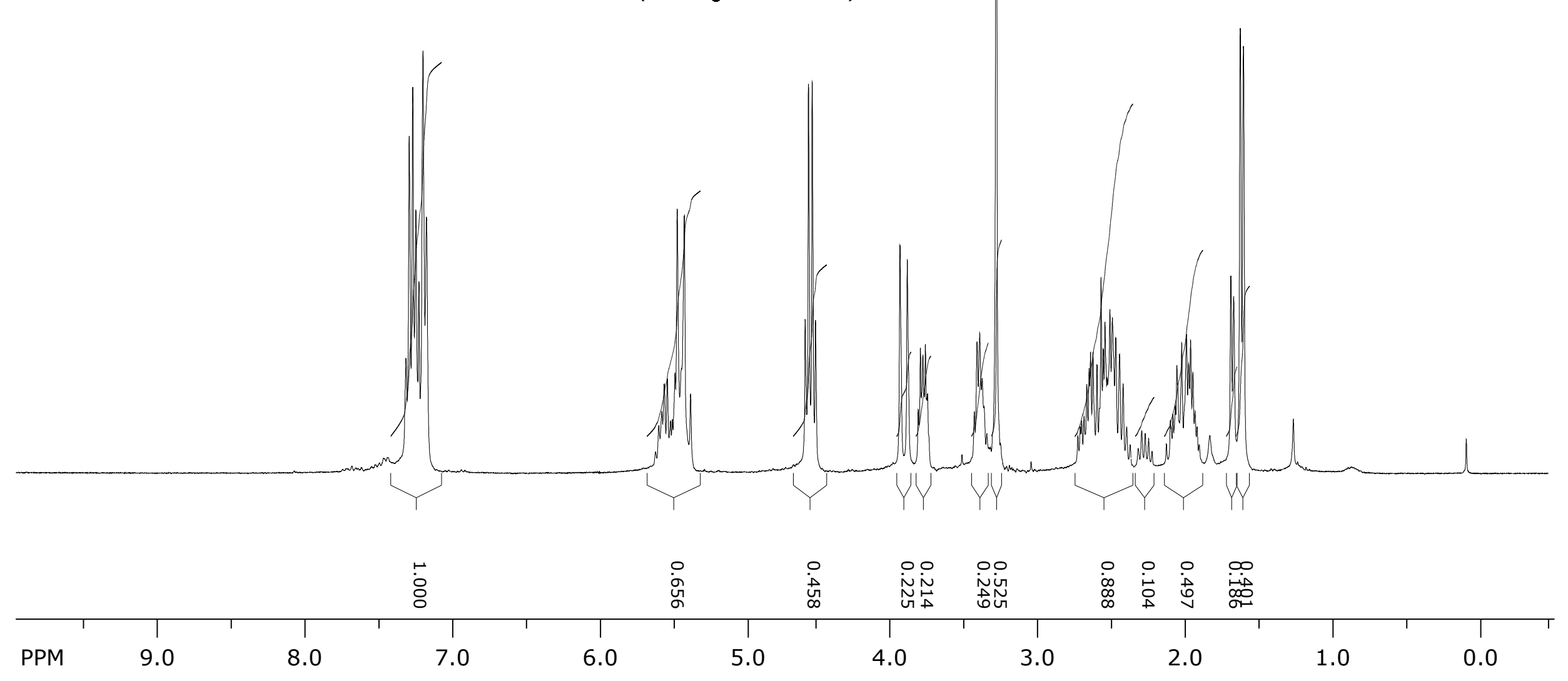



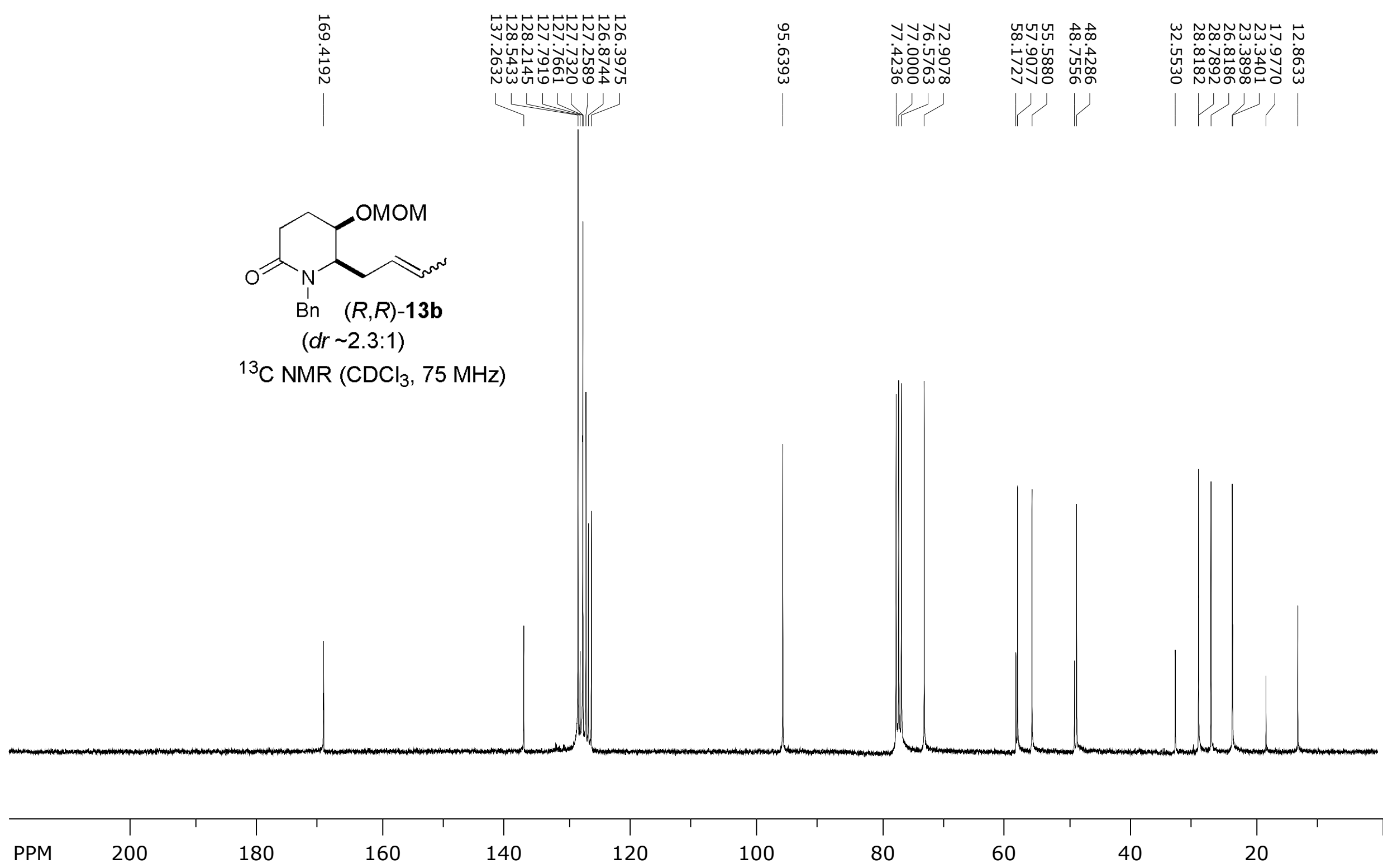


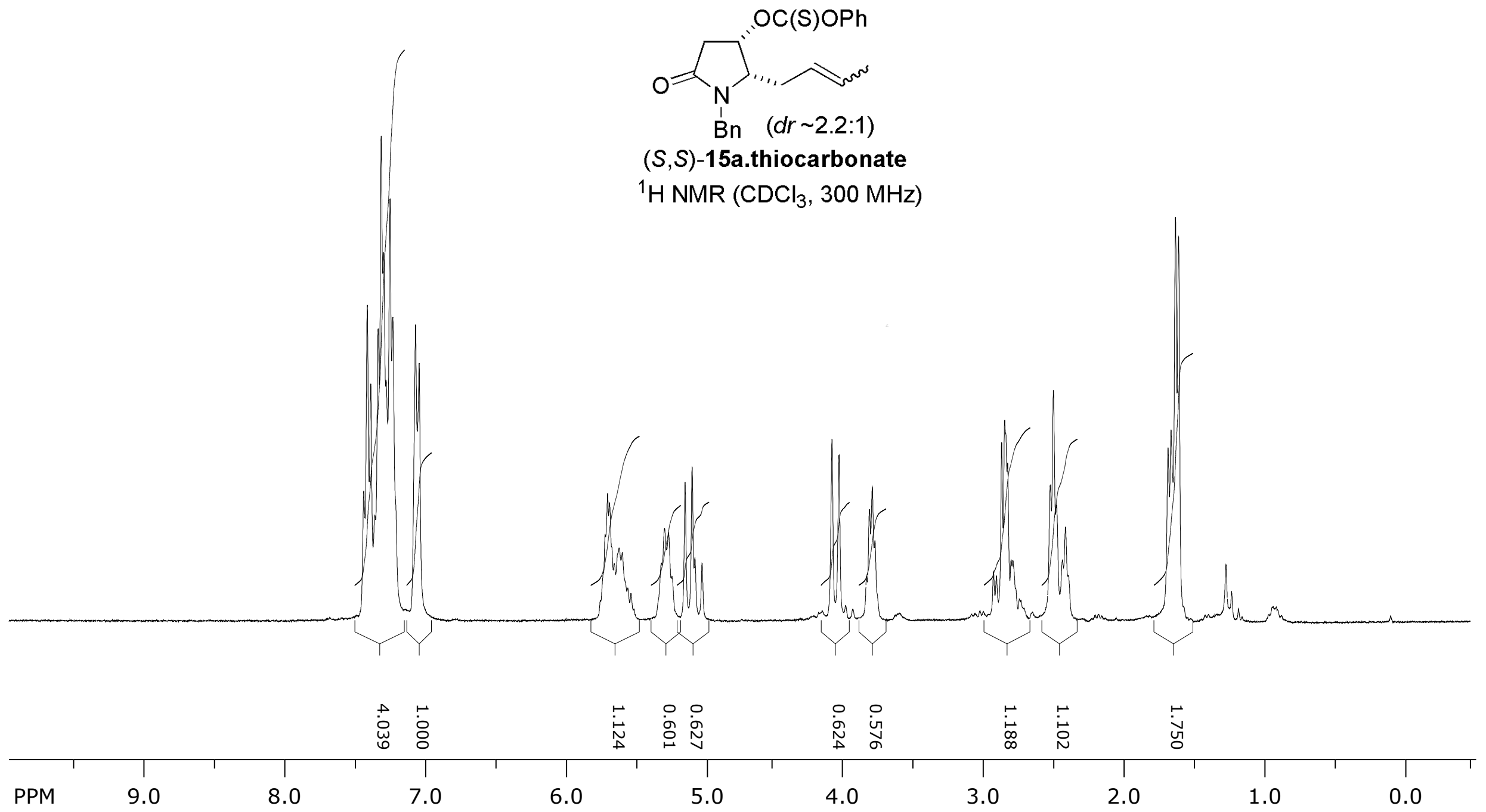

S28 

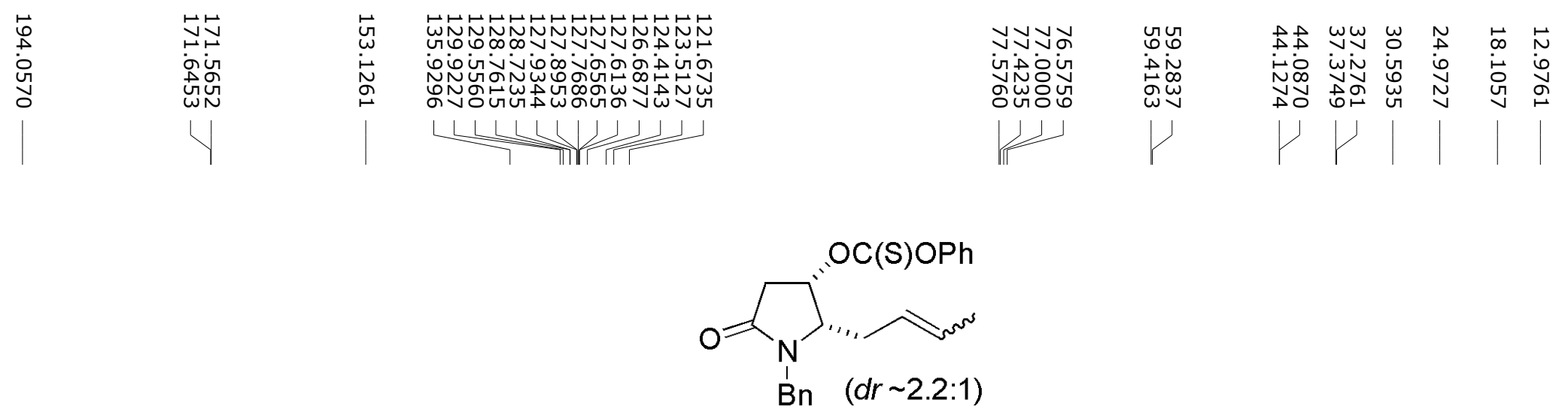

$(S, S)$-15a.thiocarbonate

${ }^{13} \mathrm{C} \mathrm{NMR}\left(\mathrm{CDCl}_{3}, 75 \mathrm{MHz}\right)$

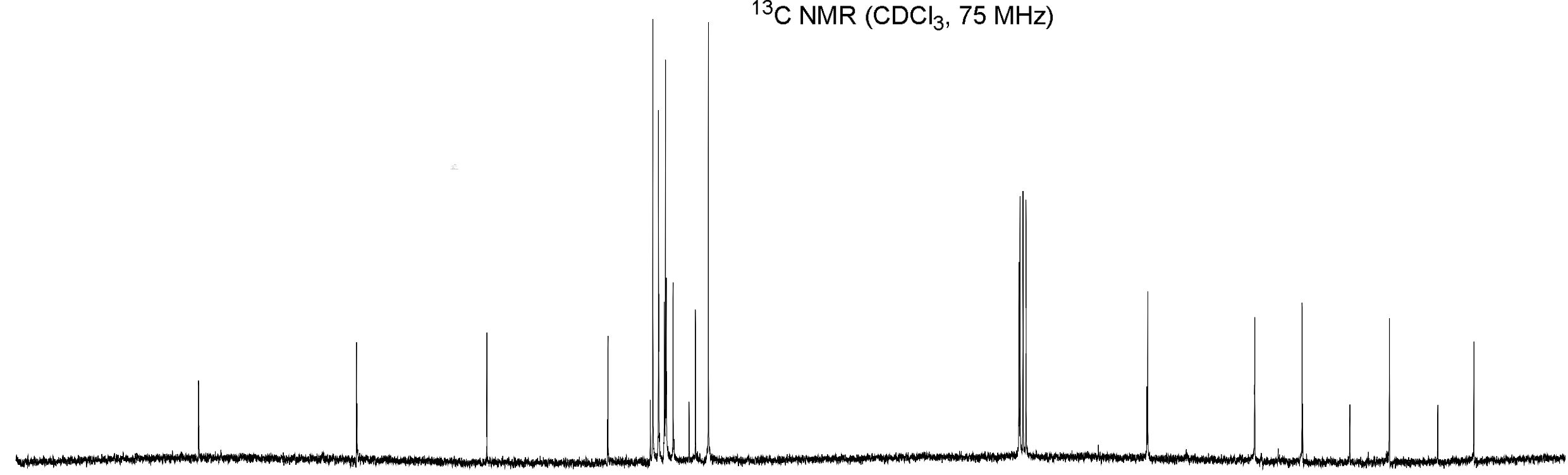




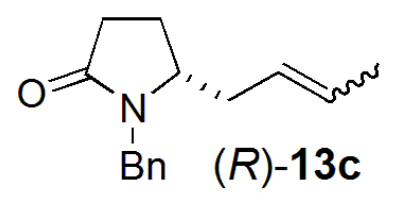

$(d r \sim 1.2: 1)$

${ }^{1} \mathrm{H} \mathrm{NMR}\left(\mathrm{CDCl}_{3}, 300 \mathrm{MHz}\right)$

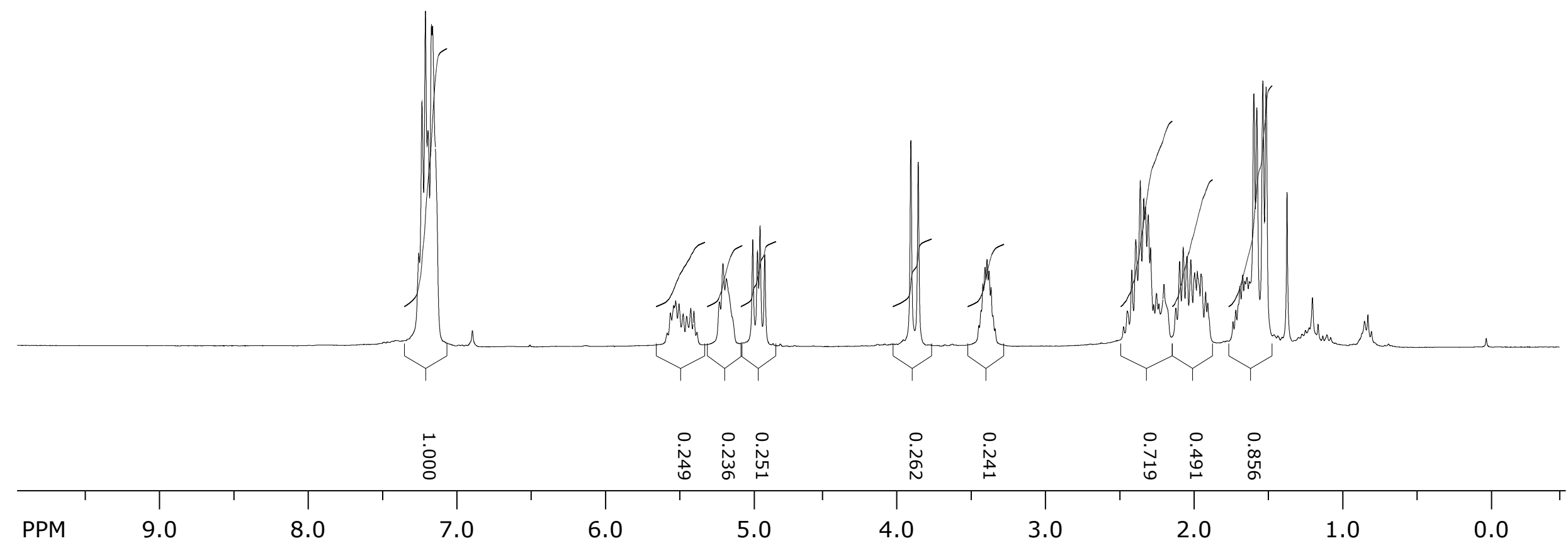




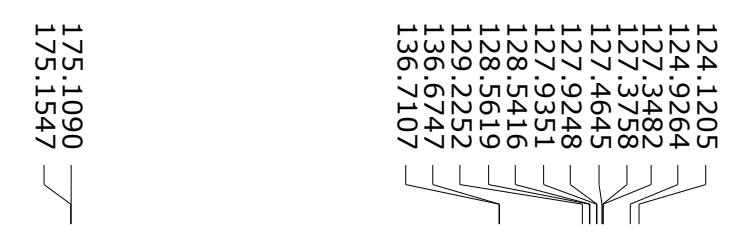

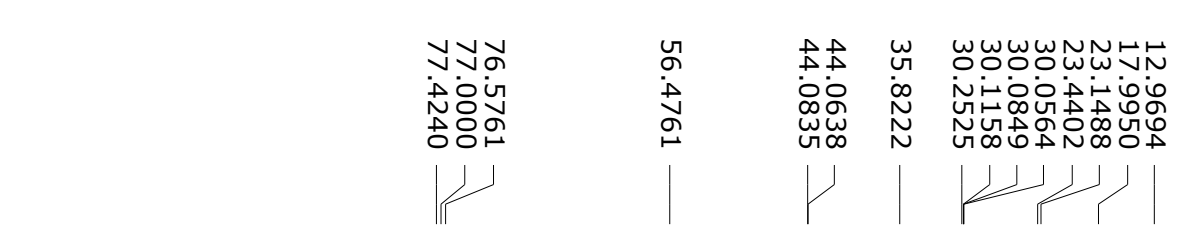

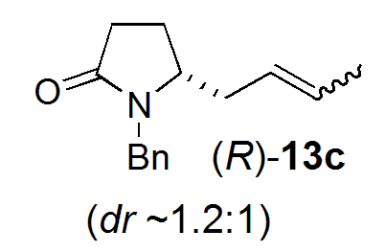

${ }^{13} \mathrm{C} \mathrm{NMR}\left(\mathrm{CDCl}_{3}, 75 \mathrm{MHz}\right)$

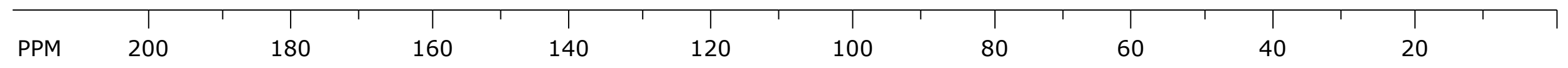




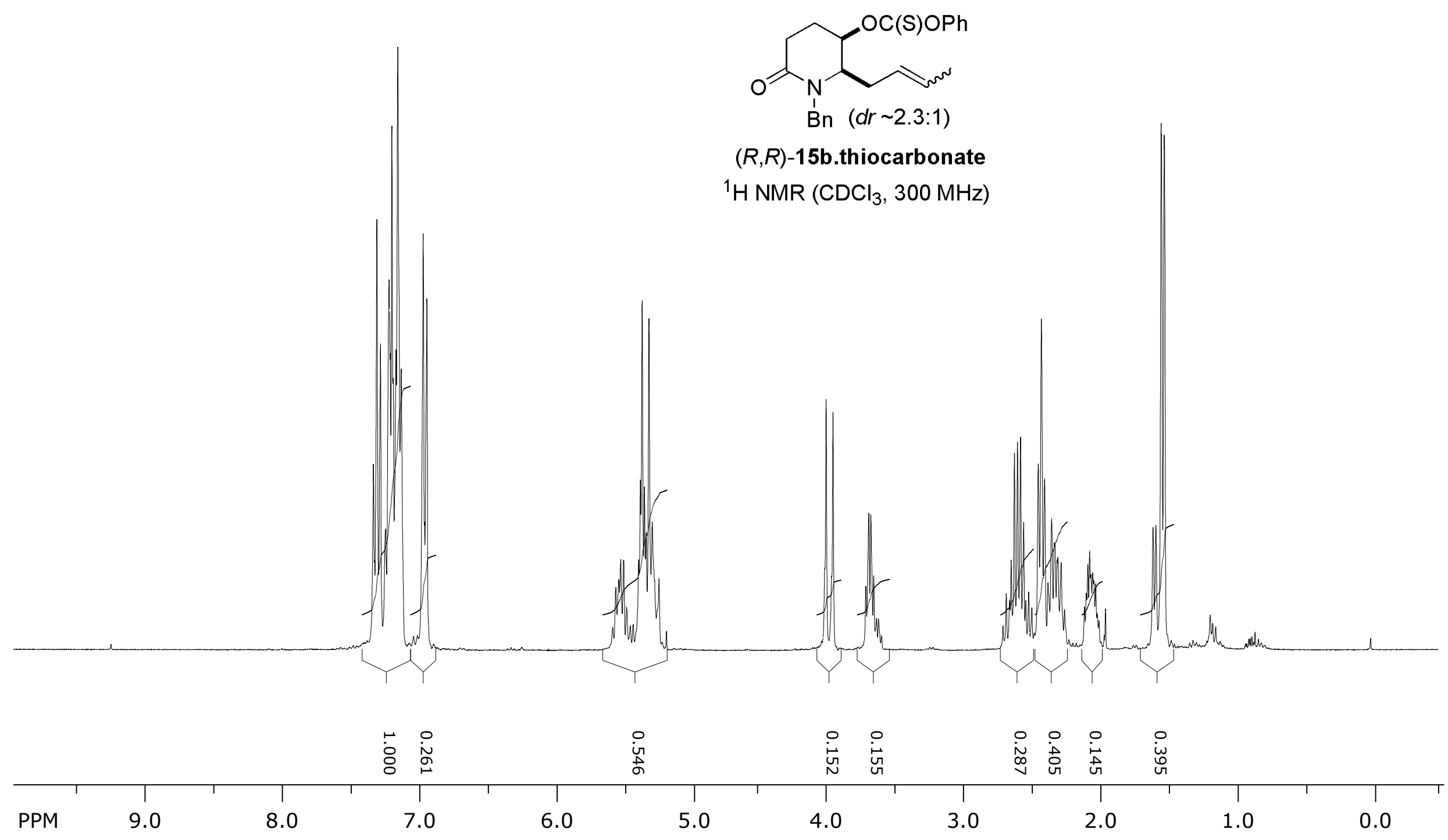




\begin{tabular}{|c|c|c|c|c|c|c|}
\hline 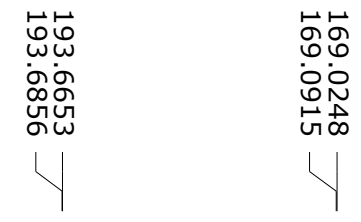 & $\begin{array}{l}\stackrel{u}{u} \\
\stackrel{.}{\circ} \\
\stackrel{\omega}{\omega}\end{array}$ & 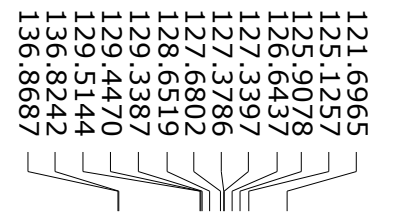 & 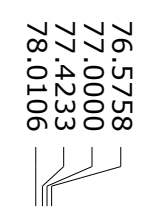 & 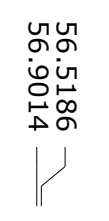 & 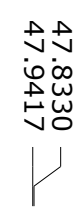 & 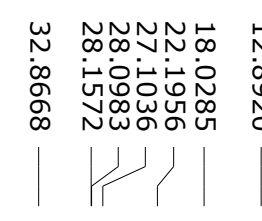 \\
\hline
\end{tabular}

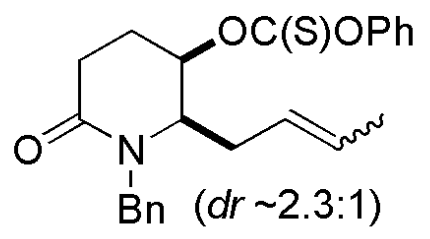

$(R, R)$-15b.thiocarbonate

${ }^{13} \mathrm{C} \mathrm{NMR}\left(\mathrm{CDCl}_{3}, 75 \mathrm{MHz}\right)$ 


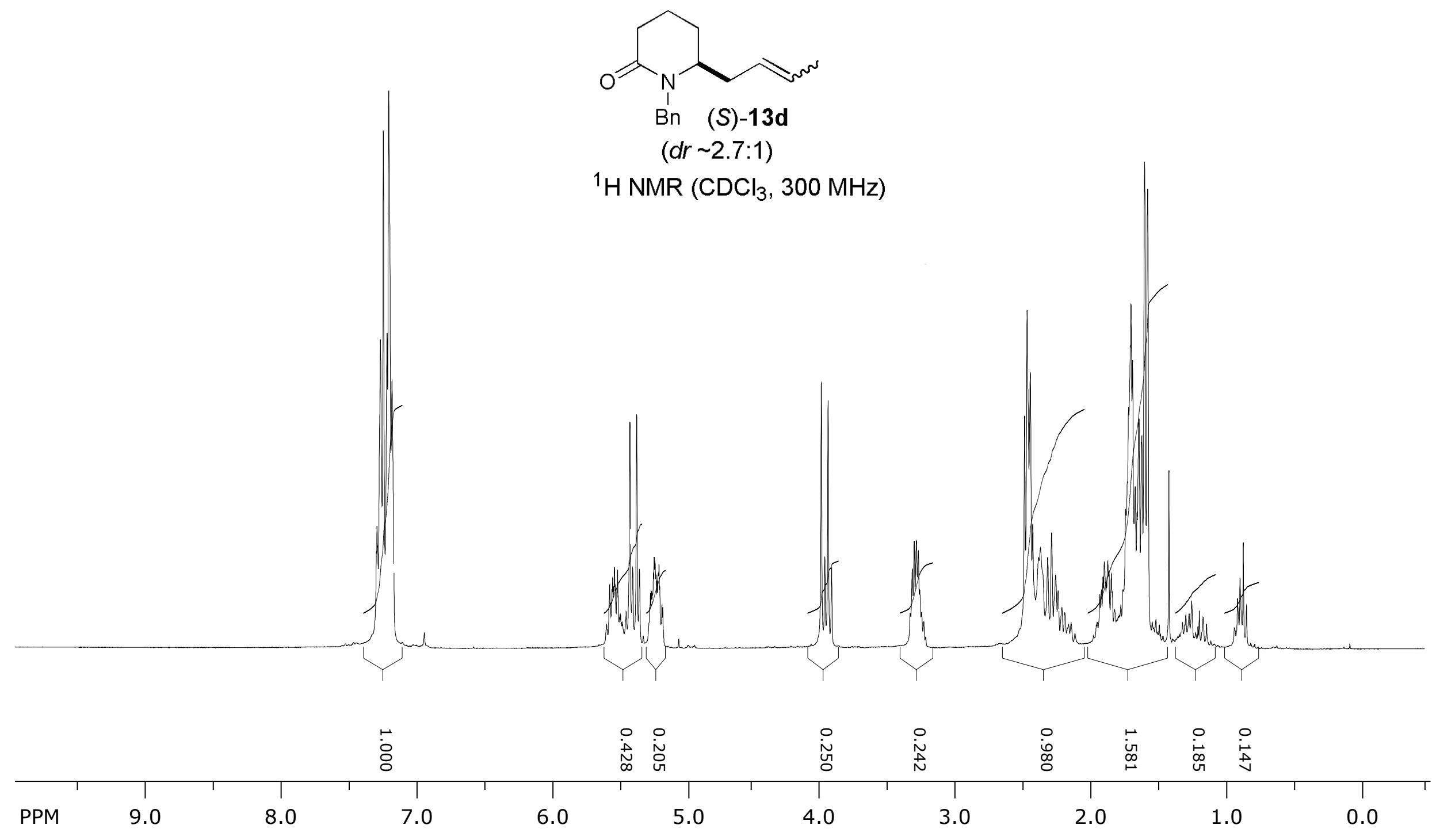




\begin{tabular}{|c|c|c|c|c|c|}
\hline 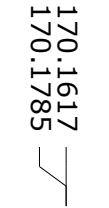 & 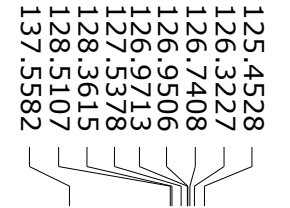 & 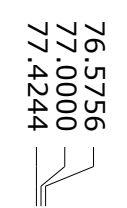 & $\begin{array}{l}\text { Un } \\
\dot{0} \\
\dot{0} \\
\stackrel{N}{0}\end{array}$ & 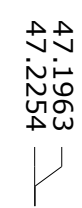 & 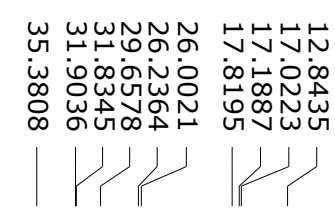 \\
\hline
\end{tabular}

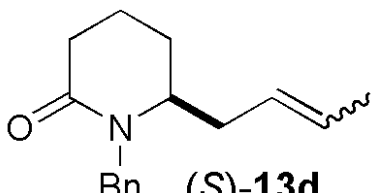

Bn (S)-13d

(dr 2.7:1)

${ }^{13} \mathrm{C} \mathrm{NMR}\left(\mathrm{CDCl}_{3}, 75 \mathrm{MHz}\right)$

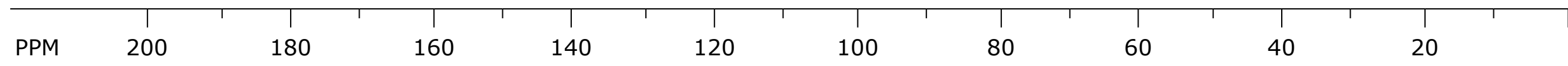




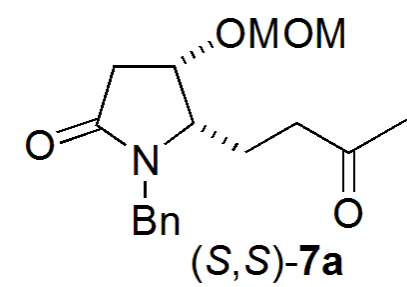

${ }^{1} \mathrm{H} \mathrm{NMR}\left(\mathrm{CDCl}_{3}, 300 \mathrm{MHz}\right)$

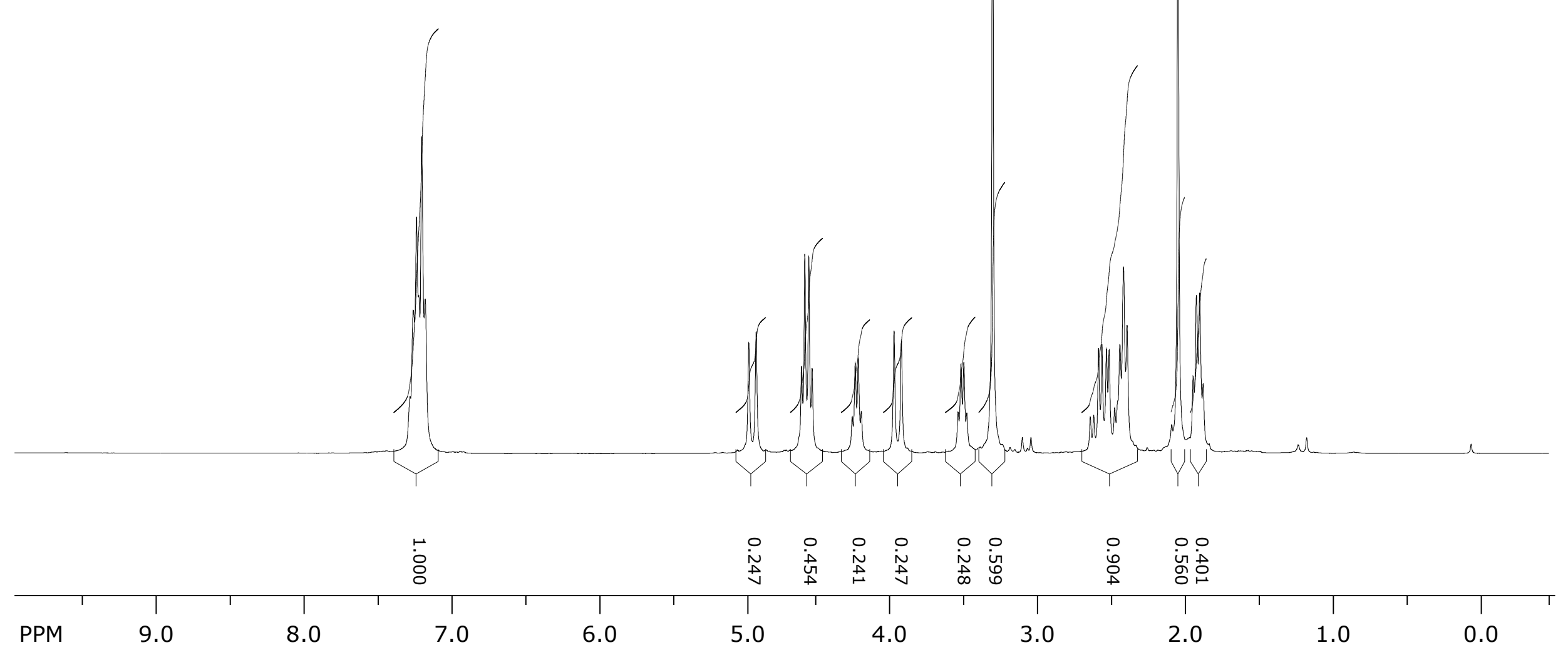




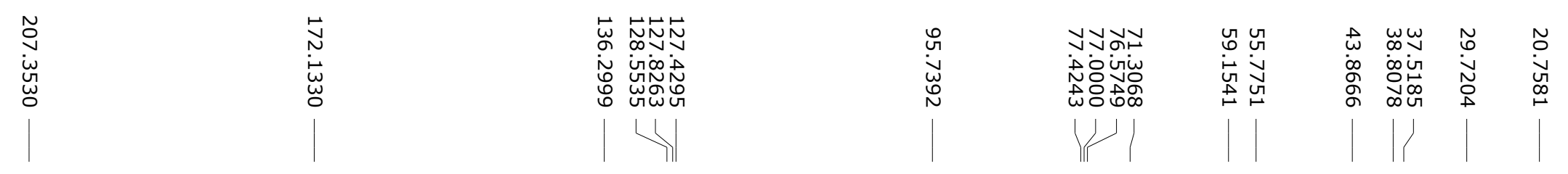

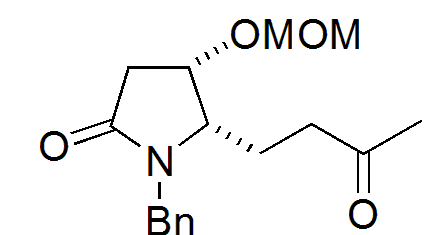

(S,S)-7a

${ }^{13} \mathrm{C} \mathrm{NMR}\left(\mathrm{CDCl}_{3}, 75 \mathrm{MHz}\right)$

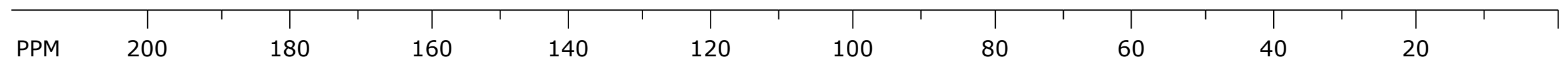




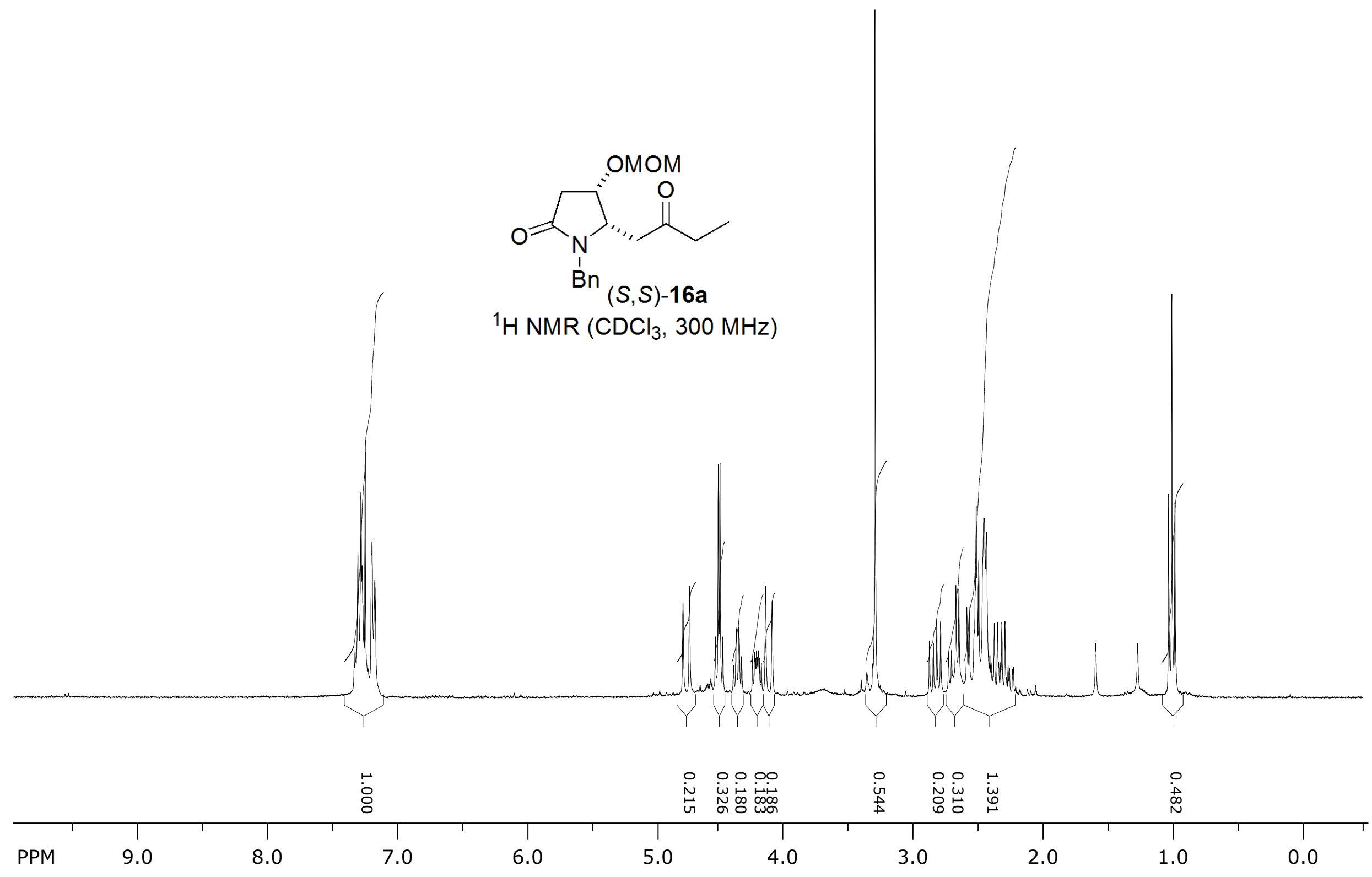




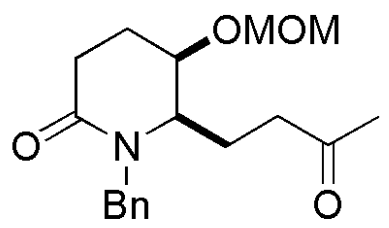

${ }^{1} \mathrm{H} \mathrm{NMR}\left(\mathrm{CDCl}_{3}, 300 \mathrm{MHz}\right)$

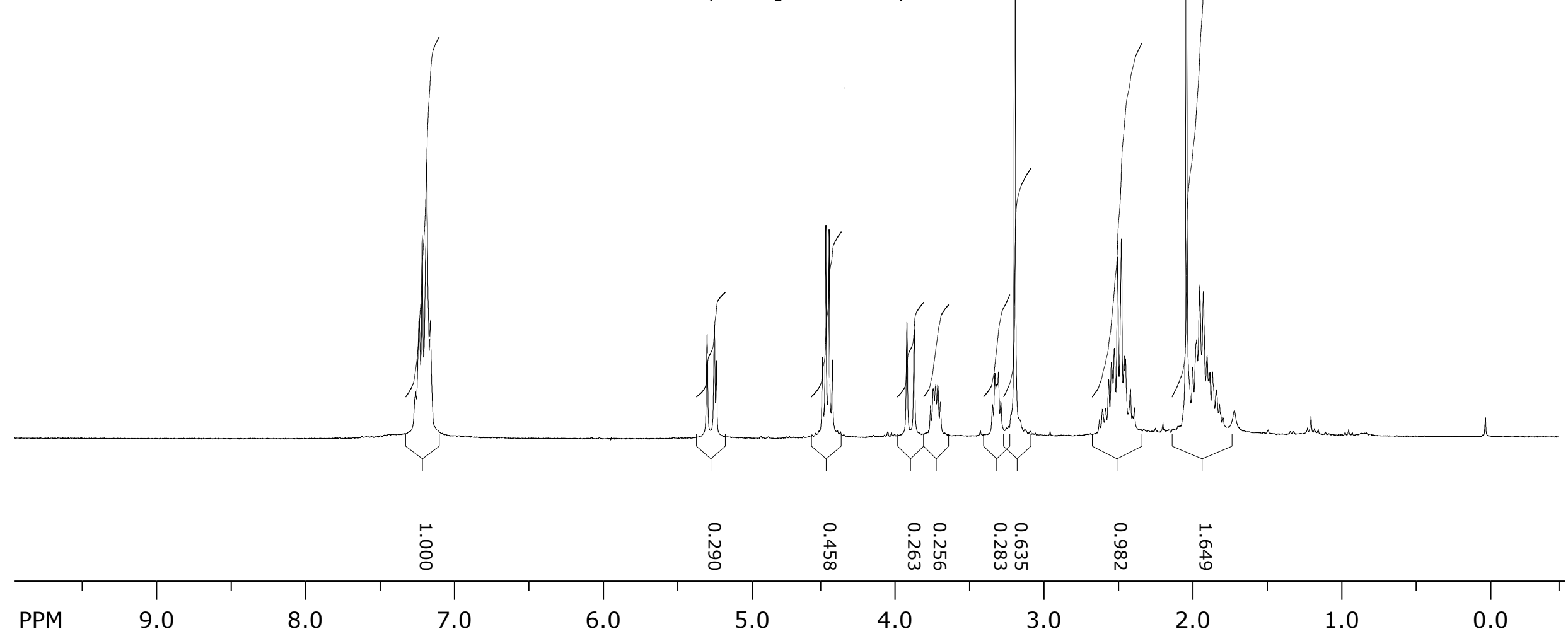




\begin{tabular}{|c|c|c|c|c|c|c|c|}
\hline 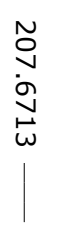 & 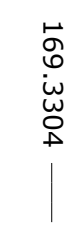 & 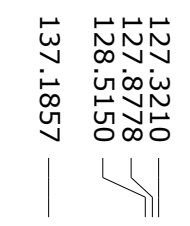 & 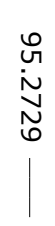 & 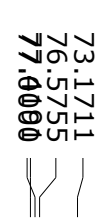 & 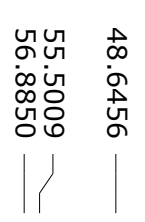 & 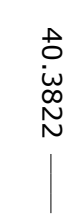 & 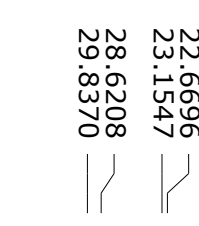 \\
\hline
\end{tabular}

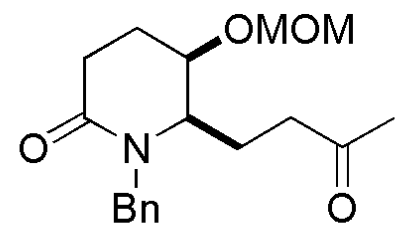

$(R, R)-7 \mathbf{b}$

${ }^{13} \mathrm{C} \mathrm{NMR}\left(\mathrm{CDCl}_{3}, 75 \mathrm{MHz}\right)$ 


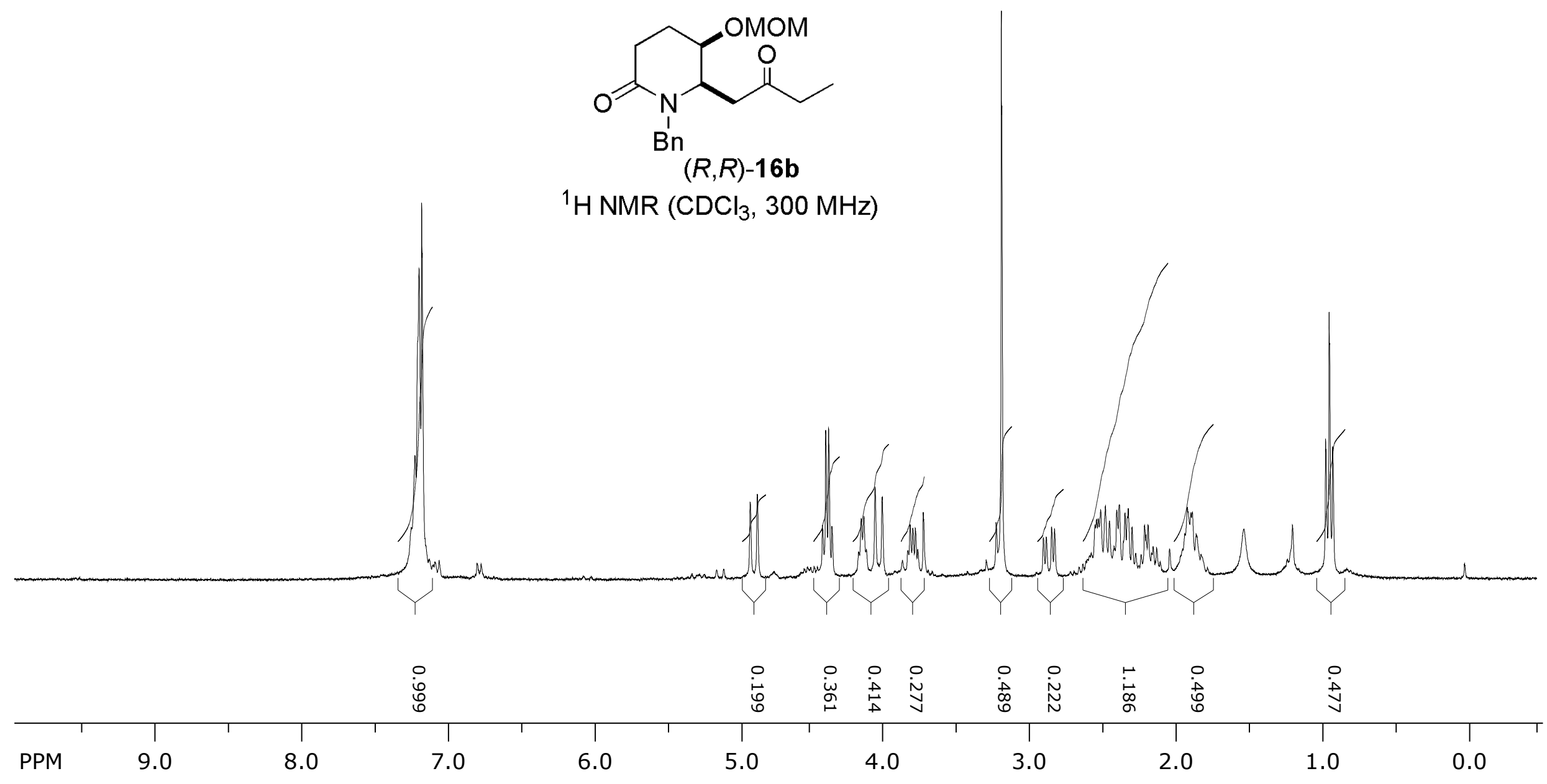




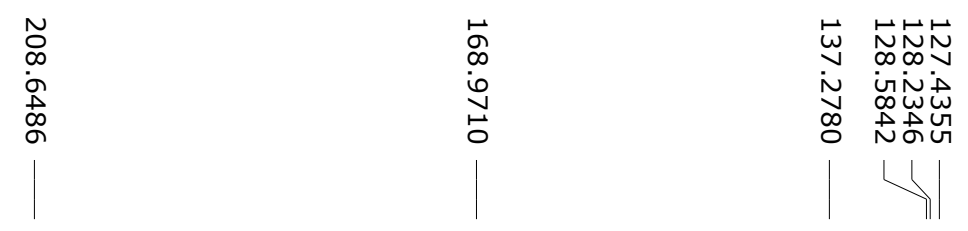

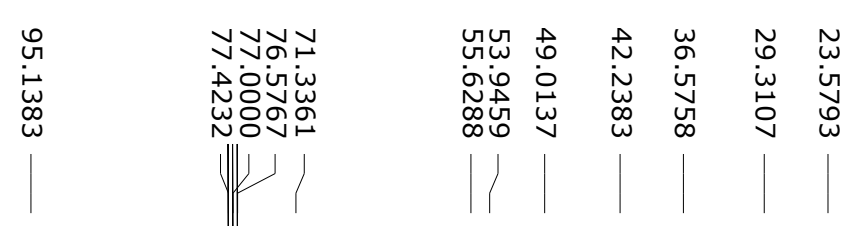

iv

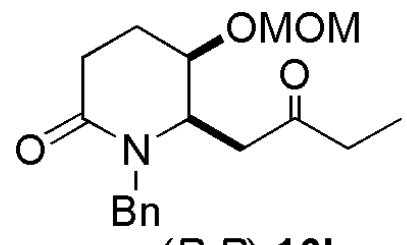

$(R, R)-\mathbf{1 6 b}$

${ }^{13} \mathrm{C} \mathrm{NMR}\left(\mathrm{CDCl}_{3}, 75 \mathrm{MHz}\right)$

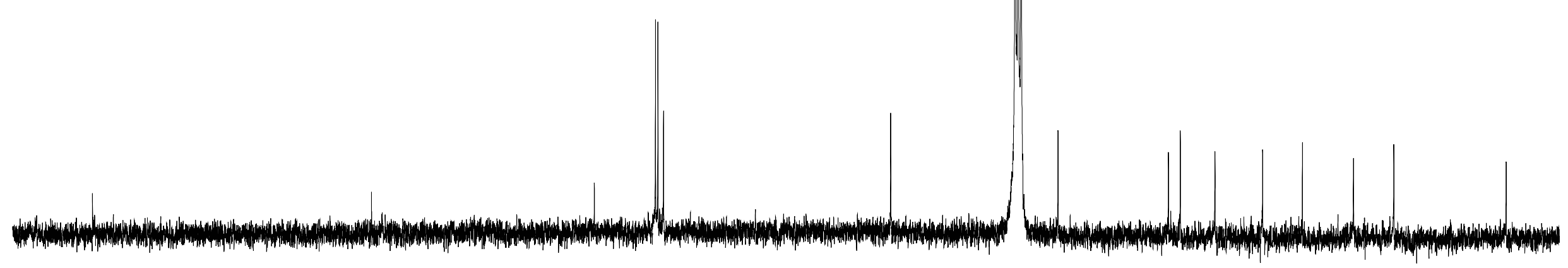




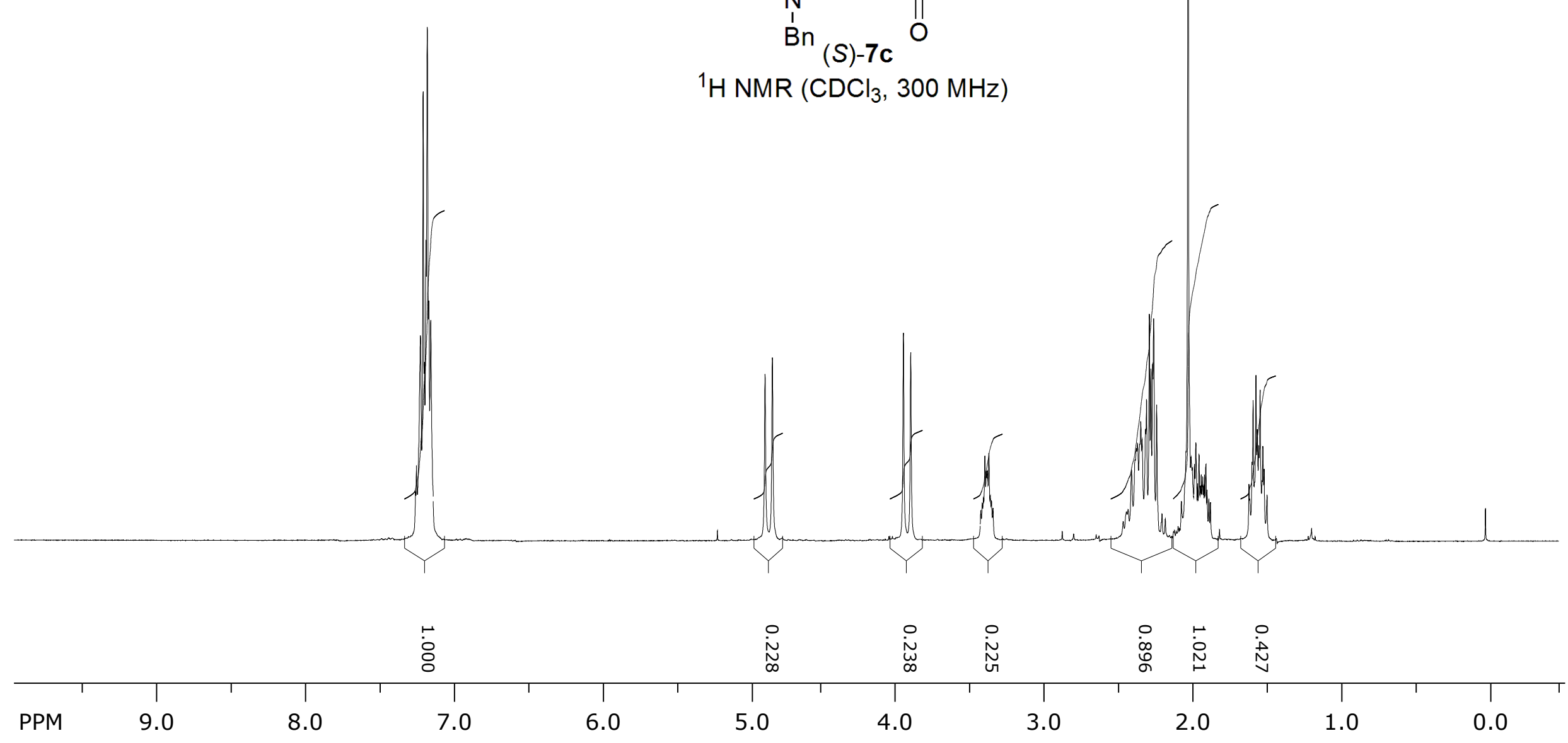



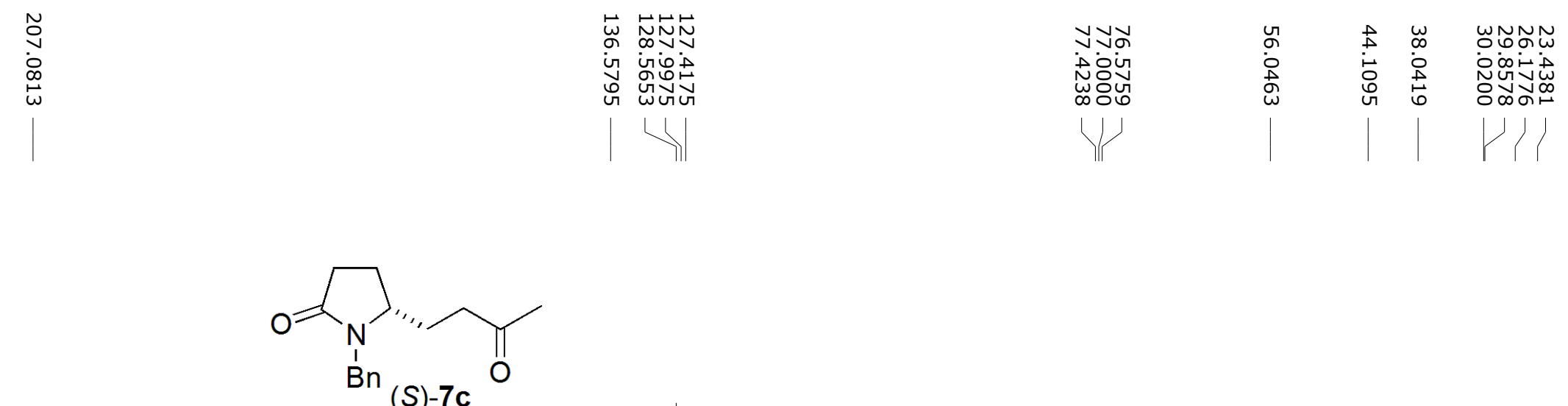

${ }^{13} \mathrm{C} \mathrm{NMR}\left(\mathrm{CDCl}_{3}, 75 \mathrm{MHz}\right)$

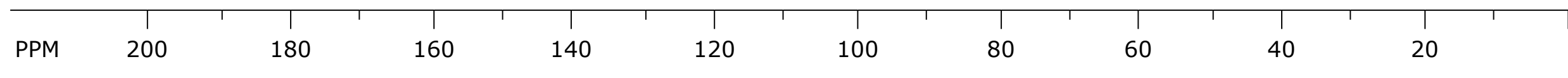




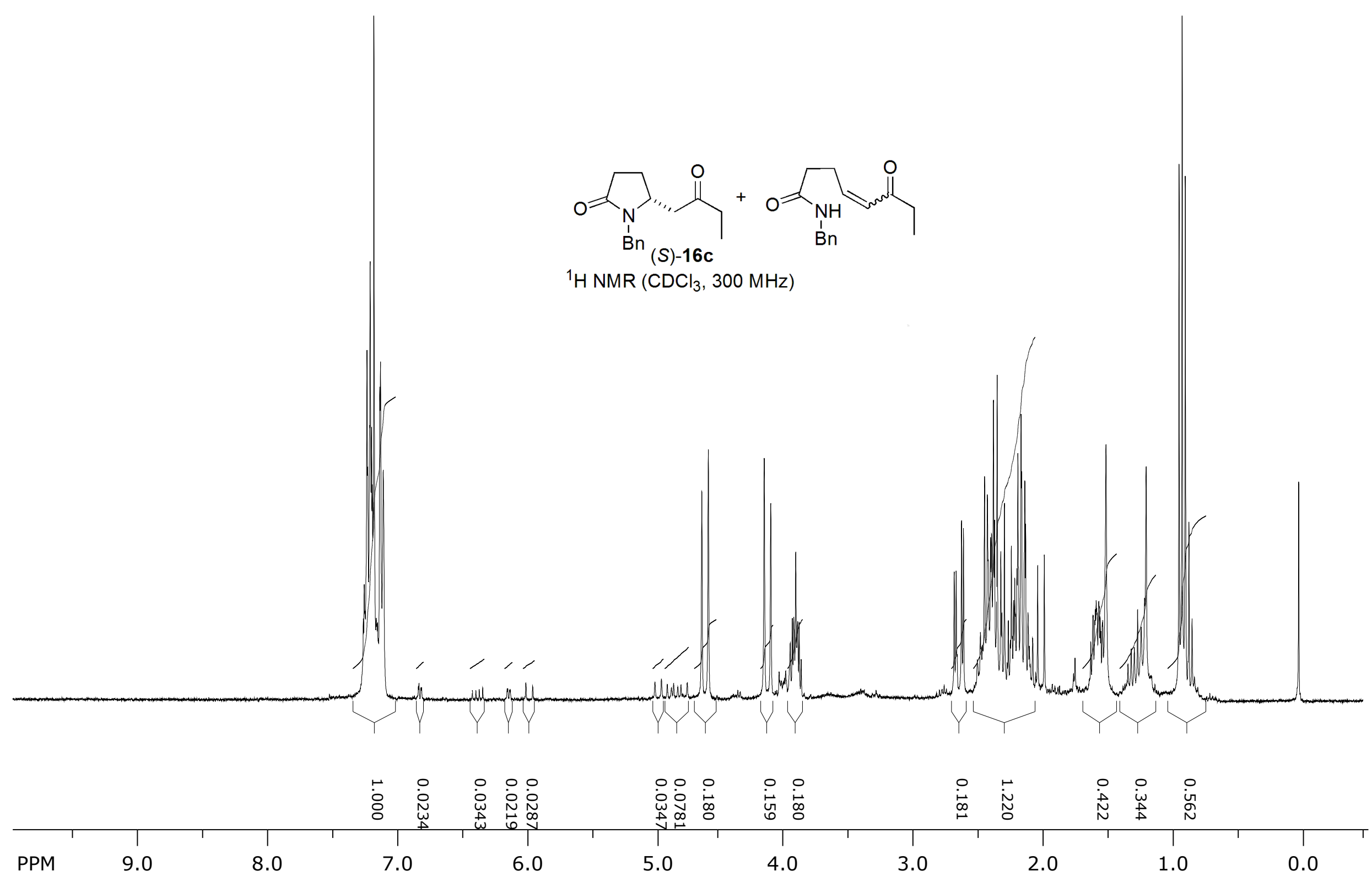




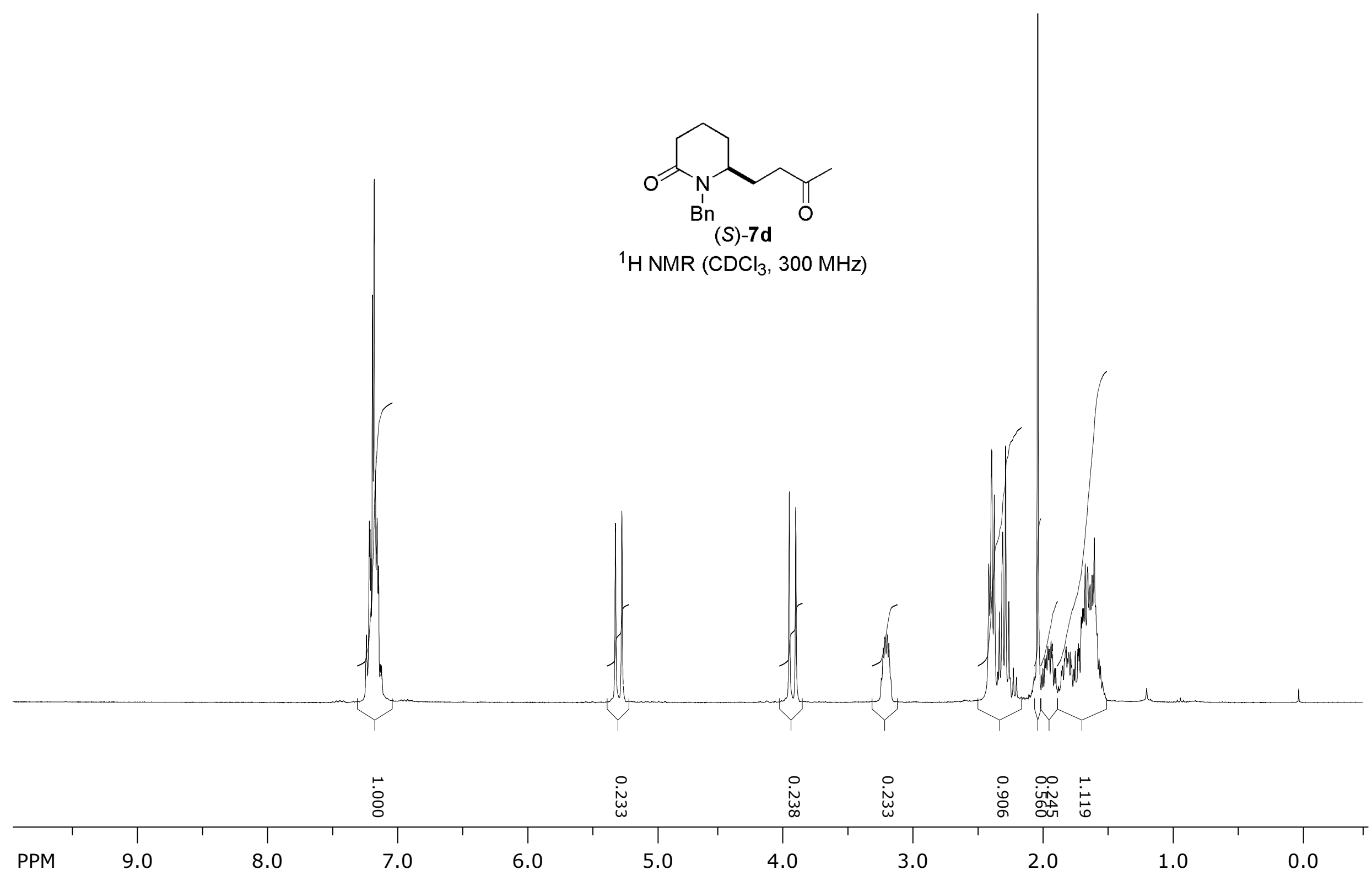




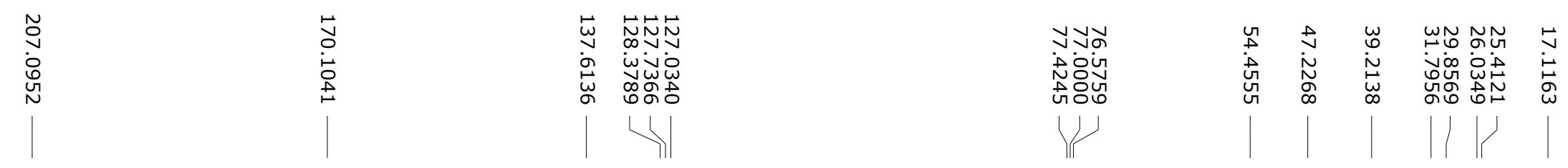

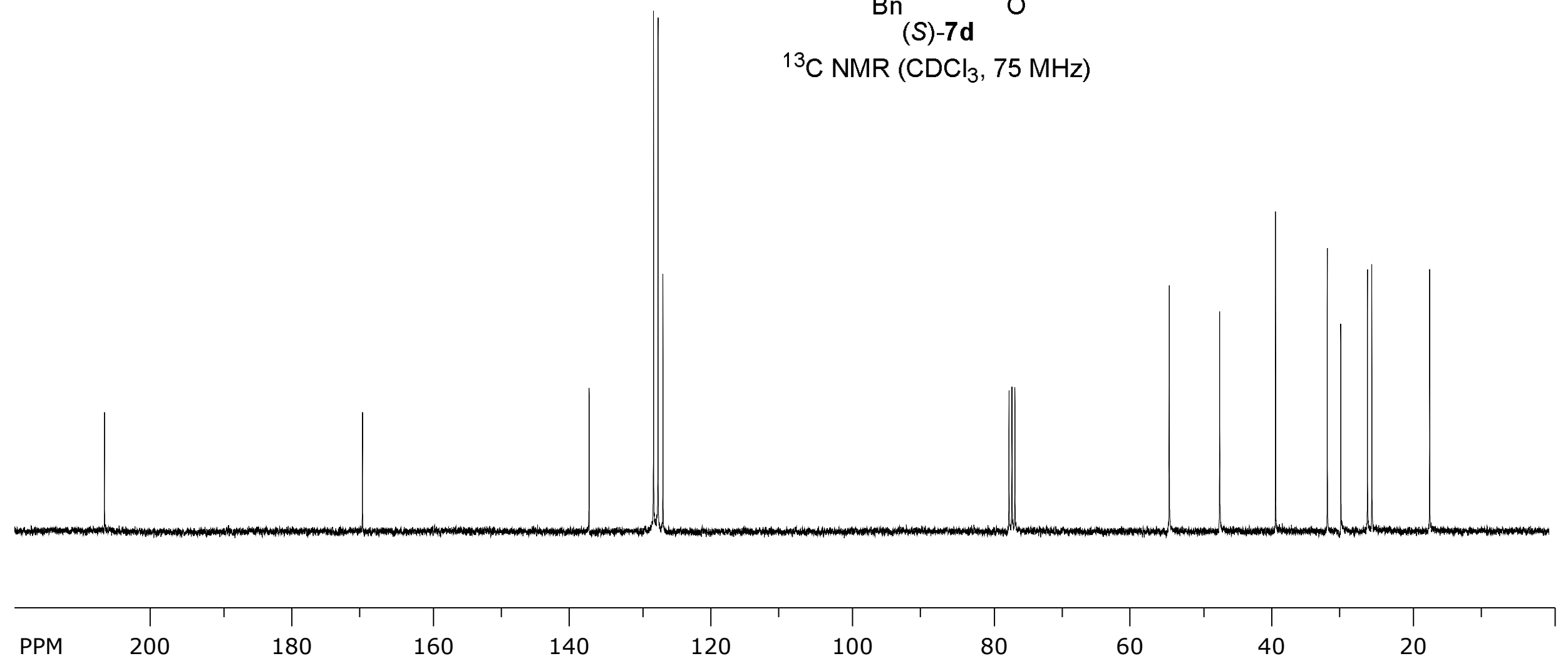




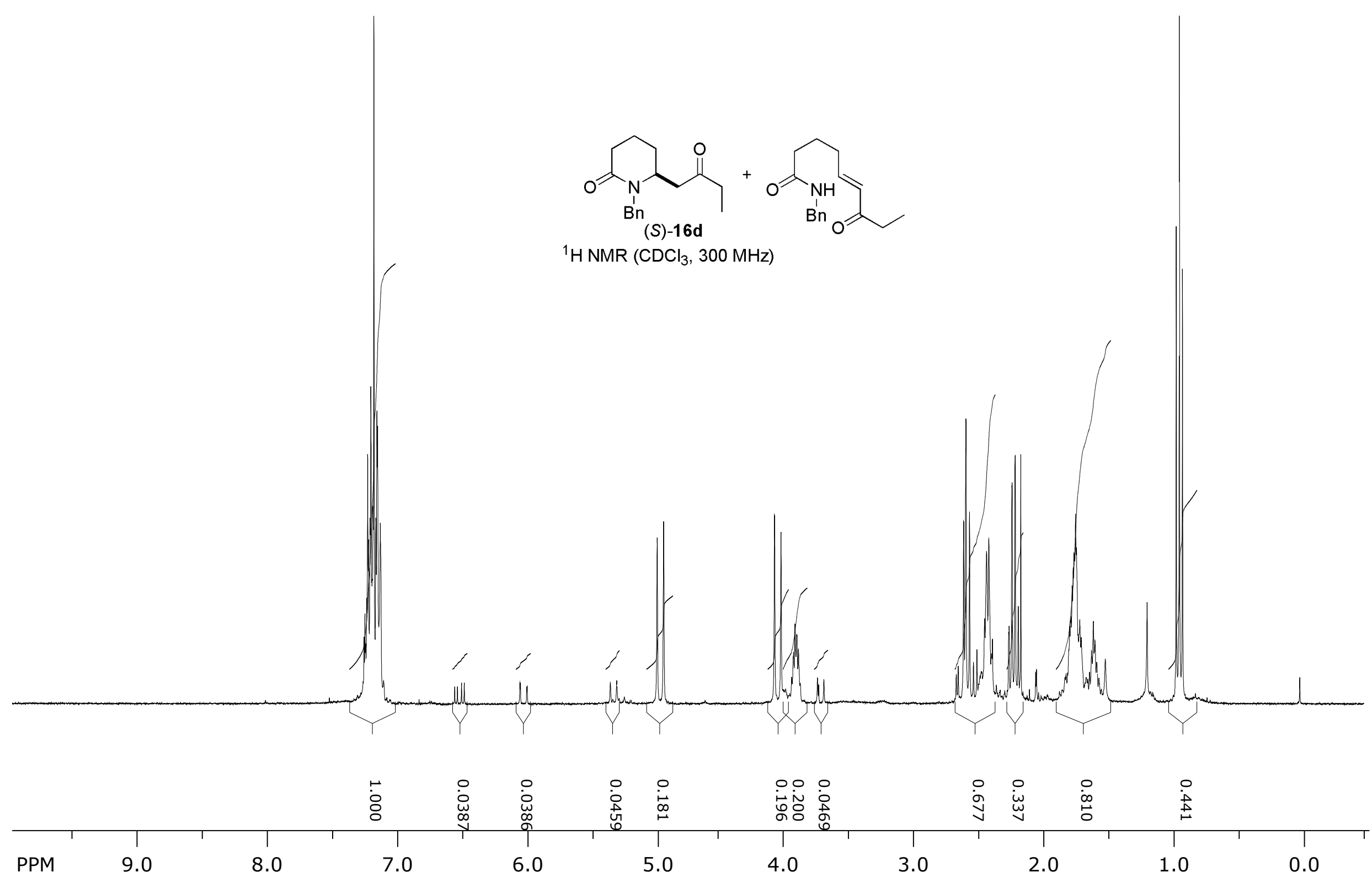




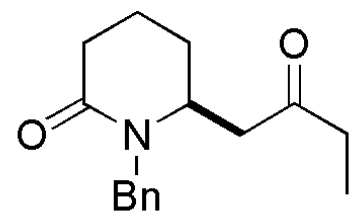

(S)-16d

${ }^{13} \mathrm{C} \mathrm{NMR}\left(\mathrm{CDCl}_{3}, 75 \mathrm{MHz}\right)$

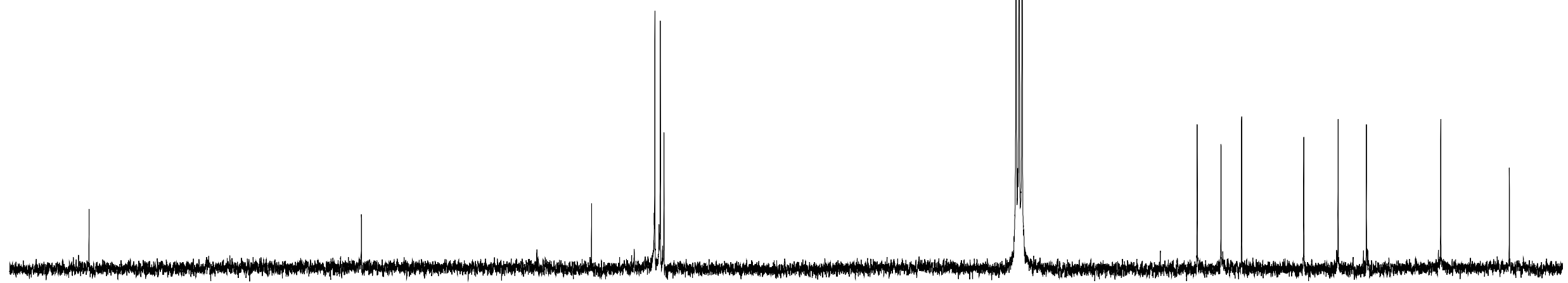




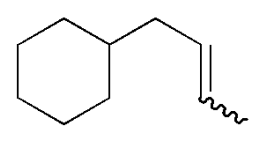

17a $(Z: E \sim 5.5: 1)$

${ }^{1} \mathrm{H} \mathrm{NMR}\left(\mathrm{CDCl}_{3}, 300 \mathrm{MHz}\right)$

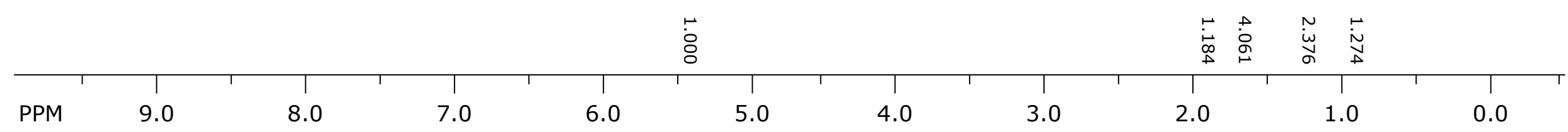




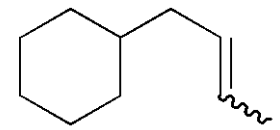

17a $(Z: E \sim 5.5: 1)$

${ }^{13} \mathrm{C} \mathrm{NMR}\left(\mathrm{CDCl}_{3}, 75 \mathrm{MHz}\right)$

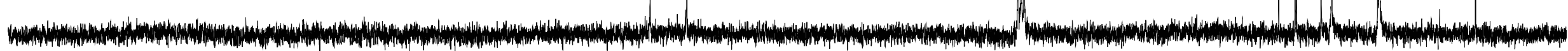




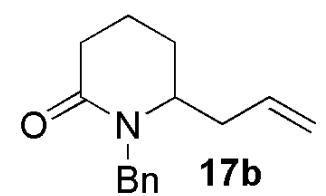

${ }^{1} \mathrm{H}$ NMR $\left(\mathrm{CDCl}_{3}, 300 \mathrm{MHz}\right)$

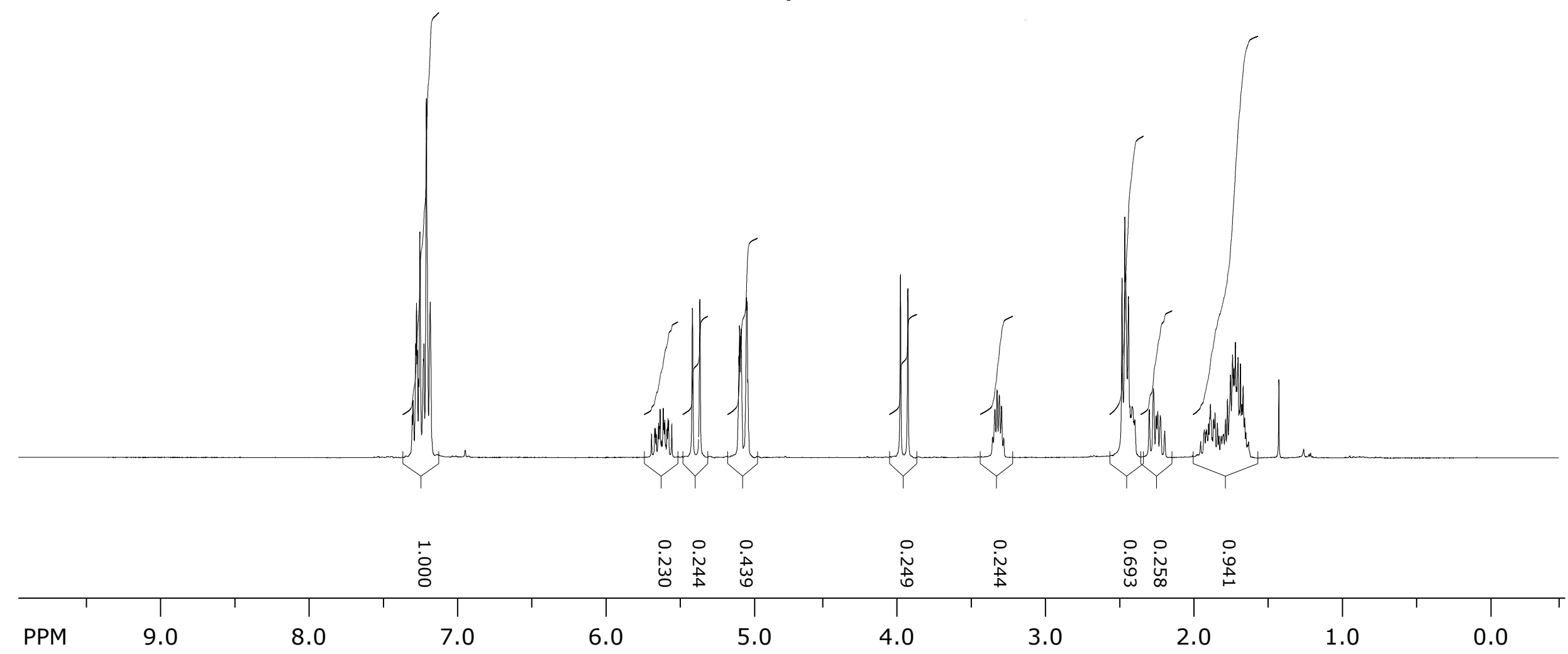




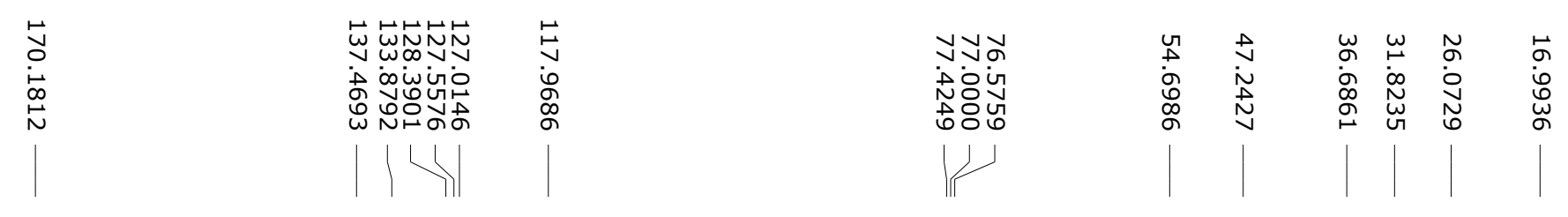

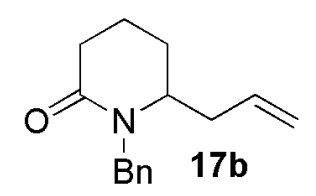

${ }^{13} \mathrm{C} \mathrm{NMR}\left(\mathrm{CDCl}_{3}, 75 \mathrm{MHz}\right)$
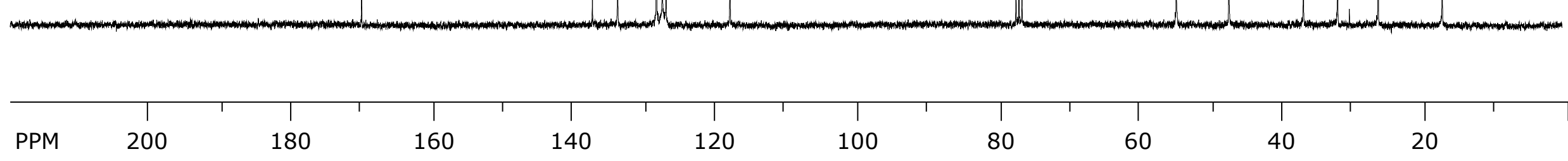


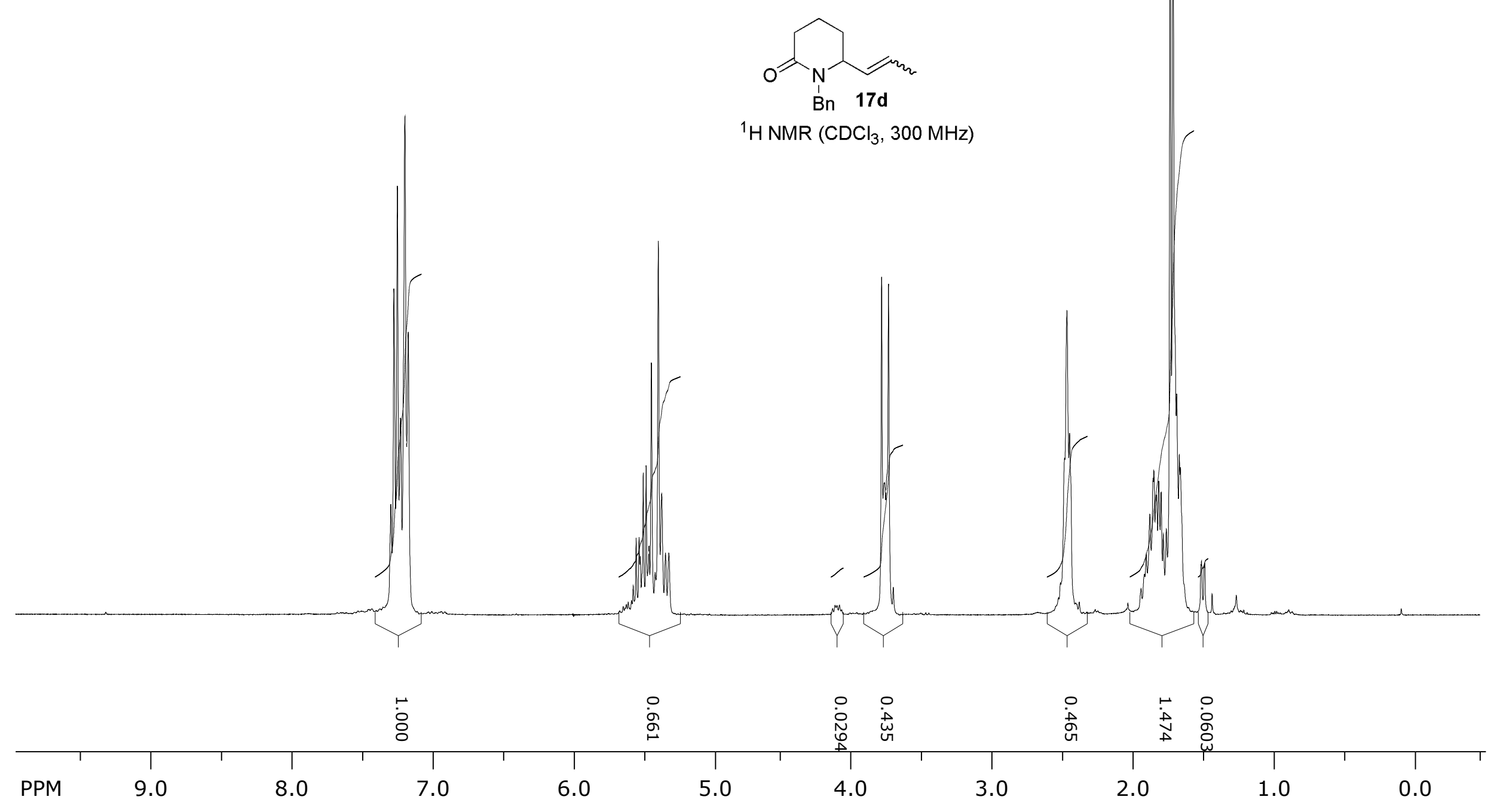




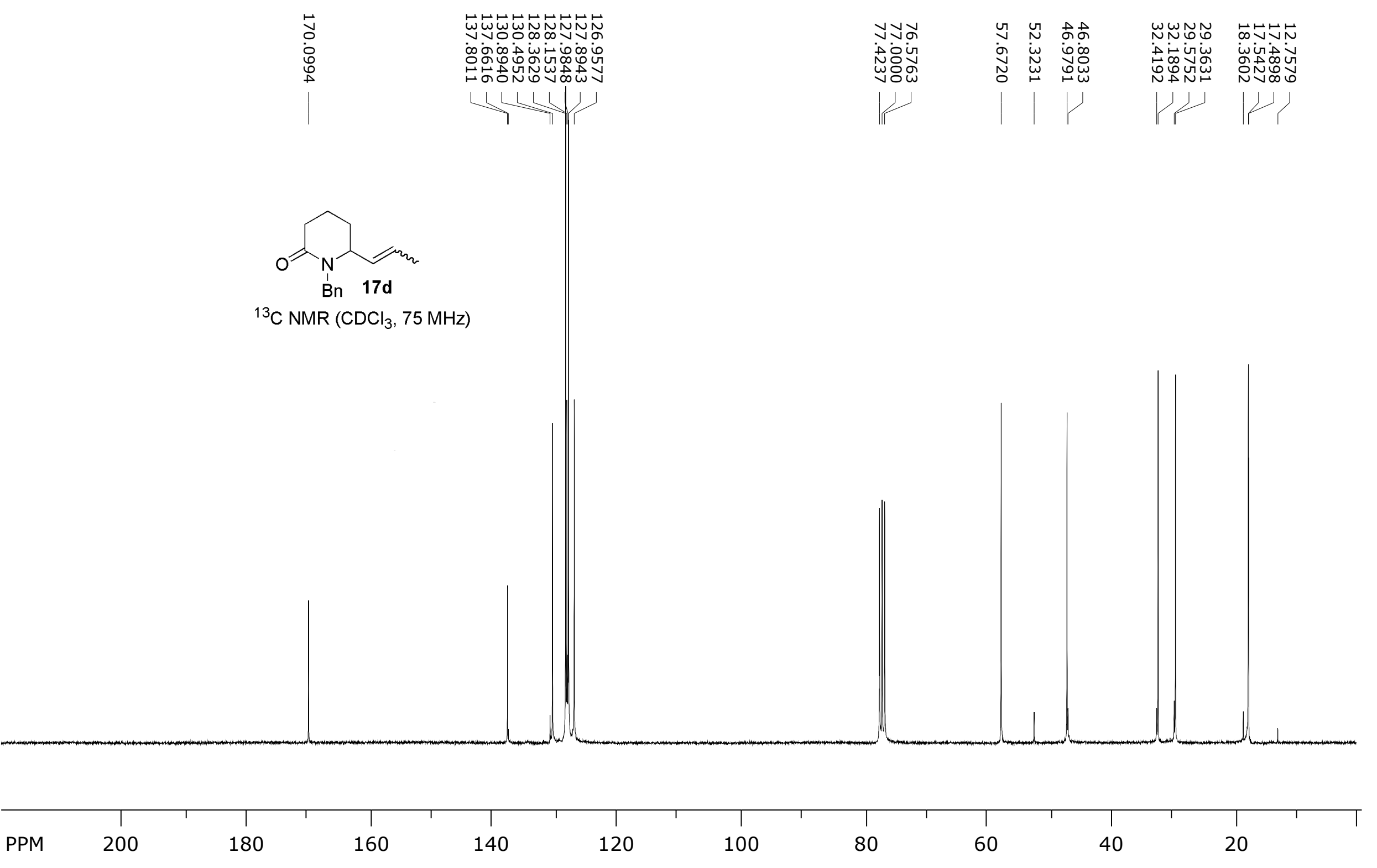




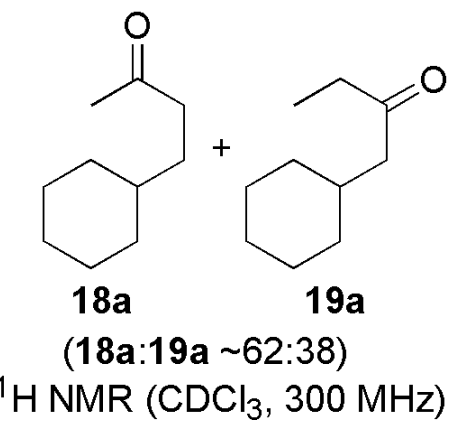

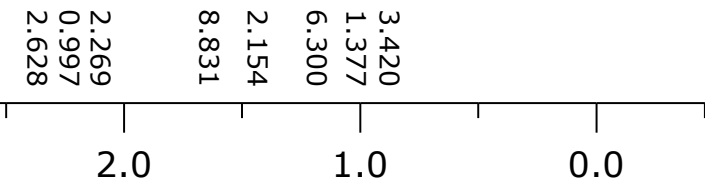




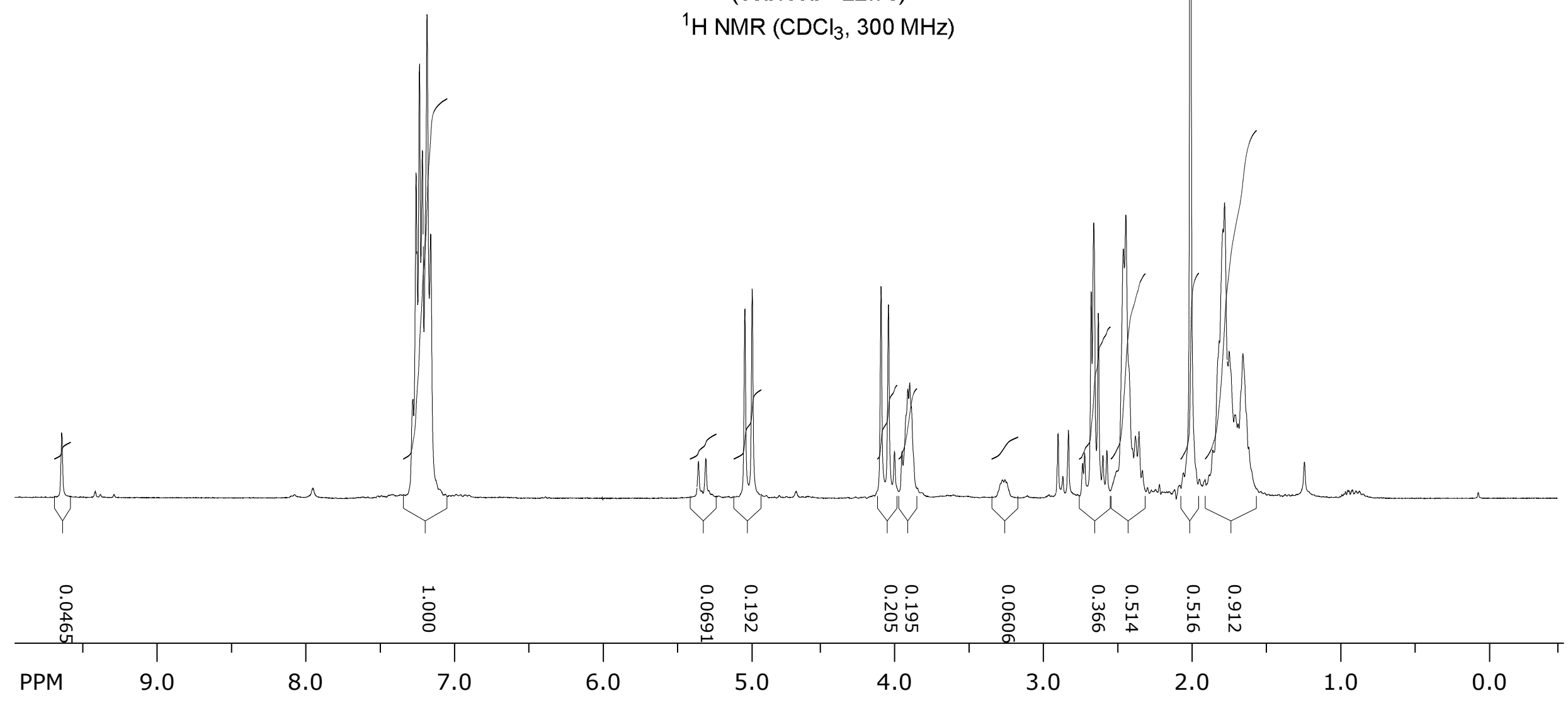



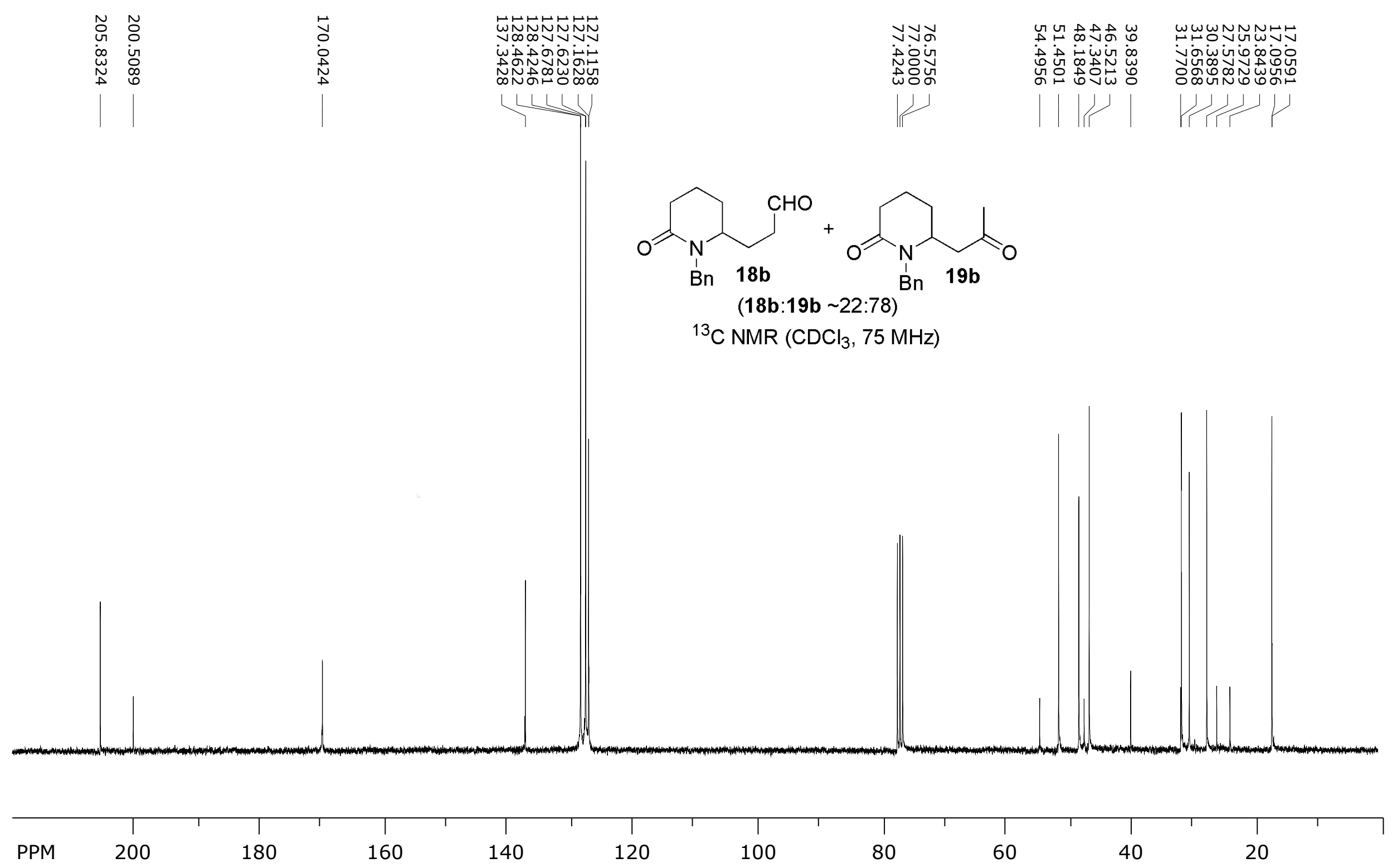


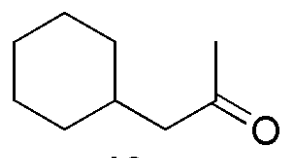

$19 \mathrm{c}$

${ }^{1} \mathrm{H} \mathrm{NMR}\left(\mathrm{CDCl}_{3}, 300 \mathrm{MHz}\right)$

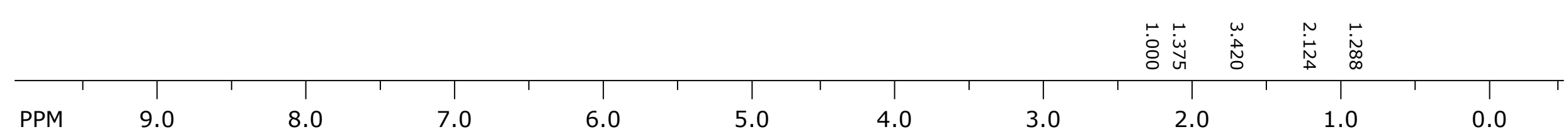




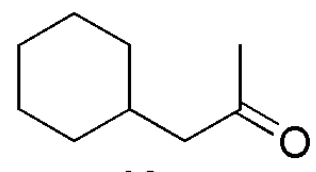

$19 \mathrm{c}$

${ }^{13} \mathrm{C} \mathrm{NMR}\left(\mathrm{CDCl}_{3}, 75 \mathrm{MHz}\right)$

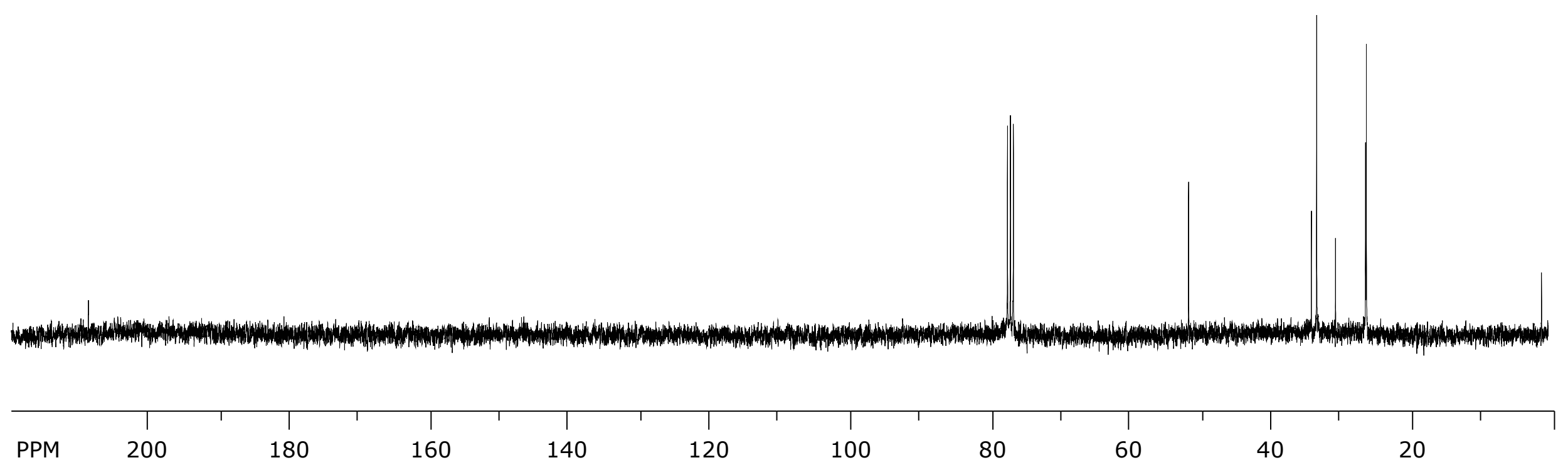




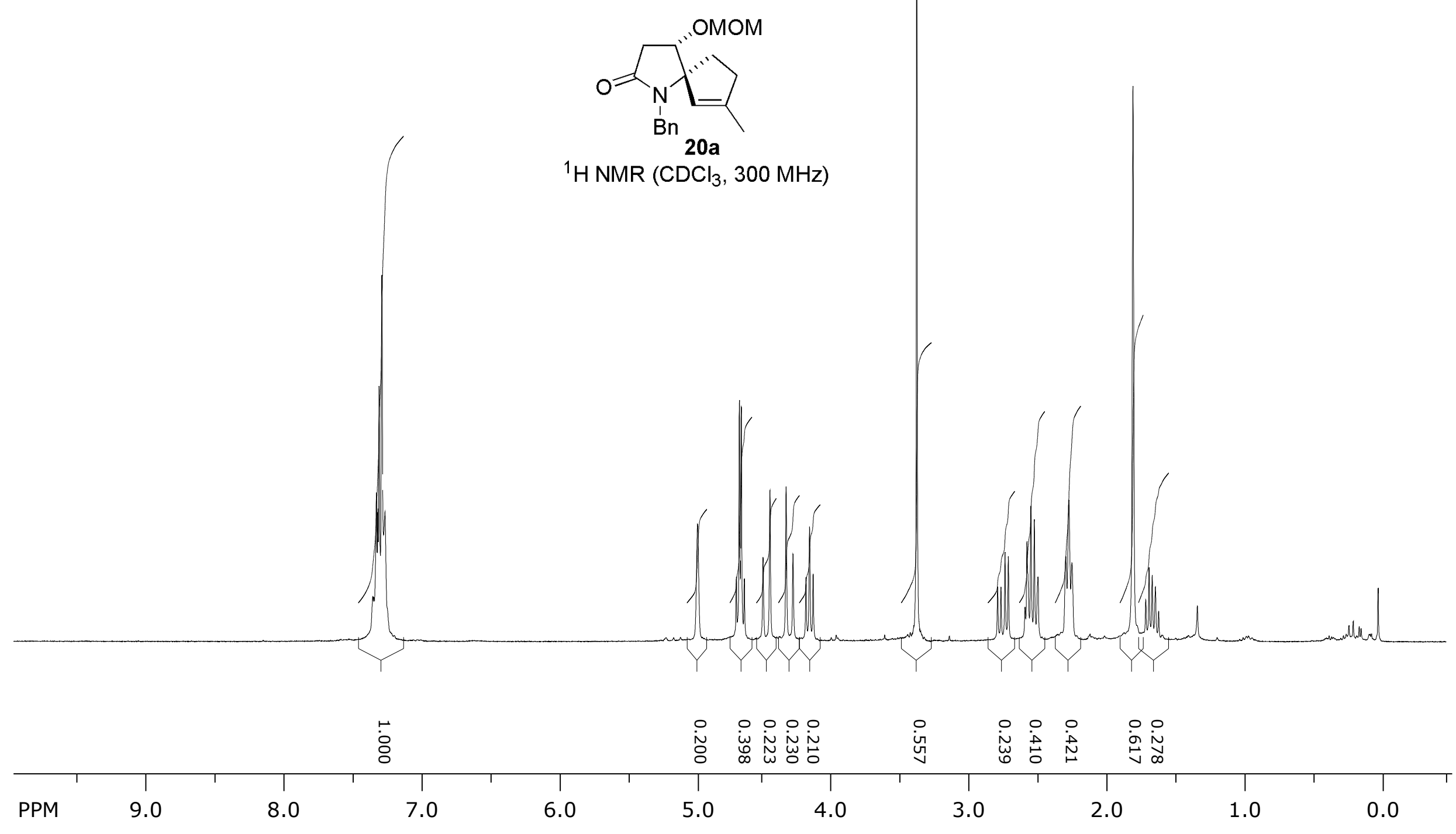



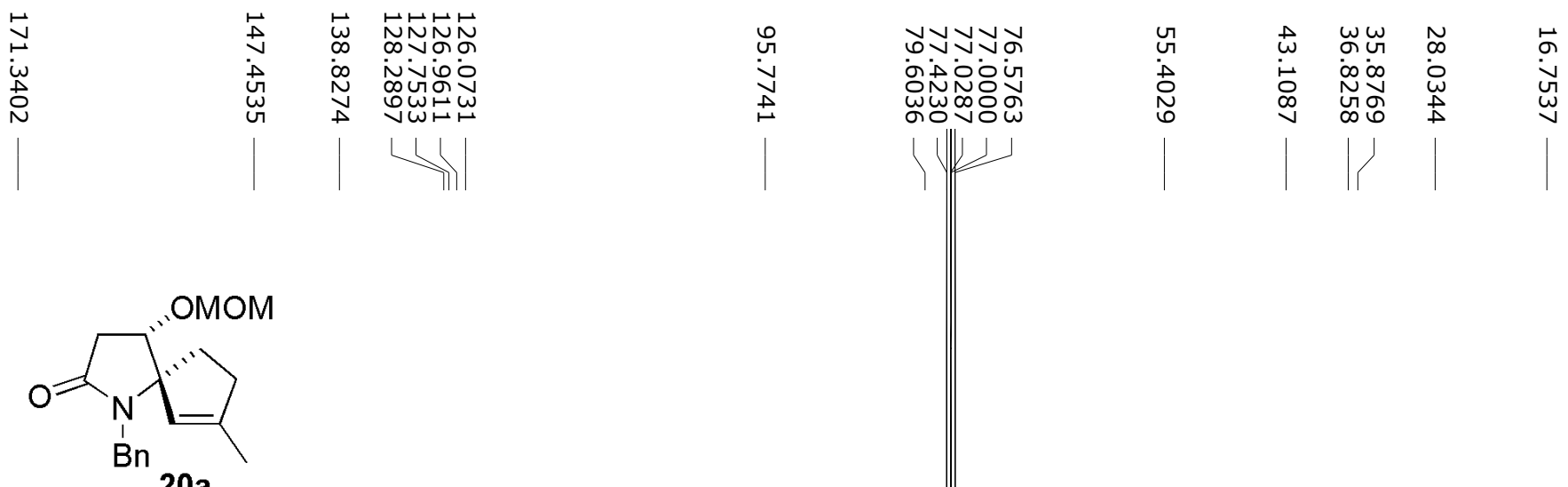

${ }^{13} \mathrm{C} \mathrm{NMR}\left(\mathrm{CDCl}_{3}, 75 \mathrm{MHz}\right)$
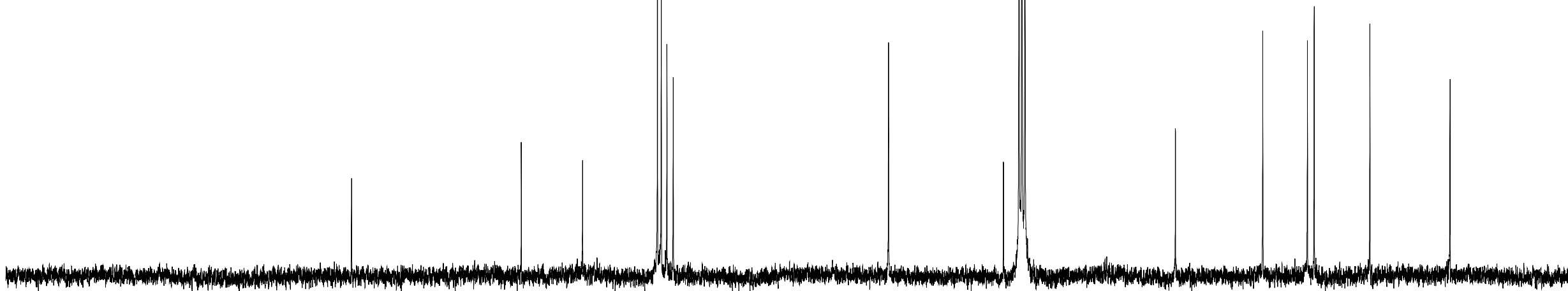


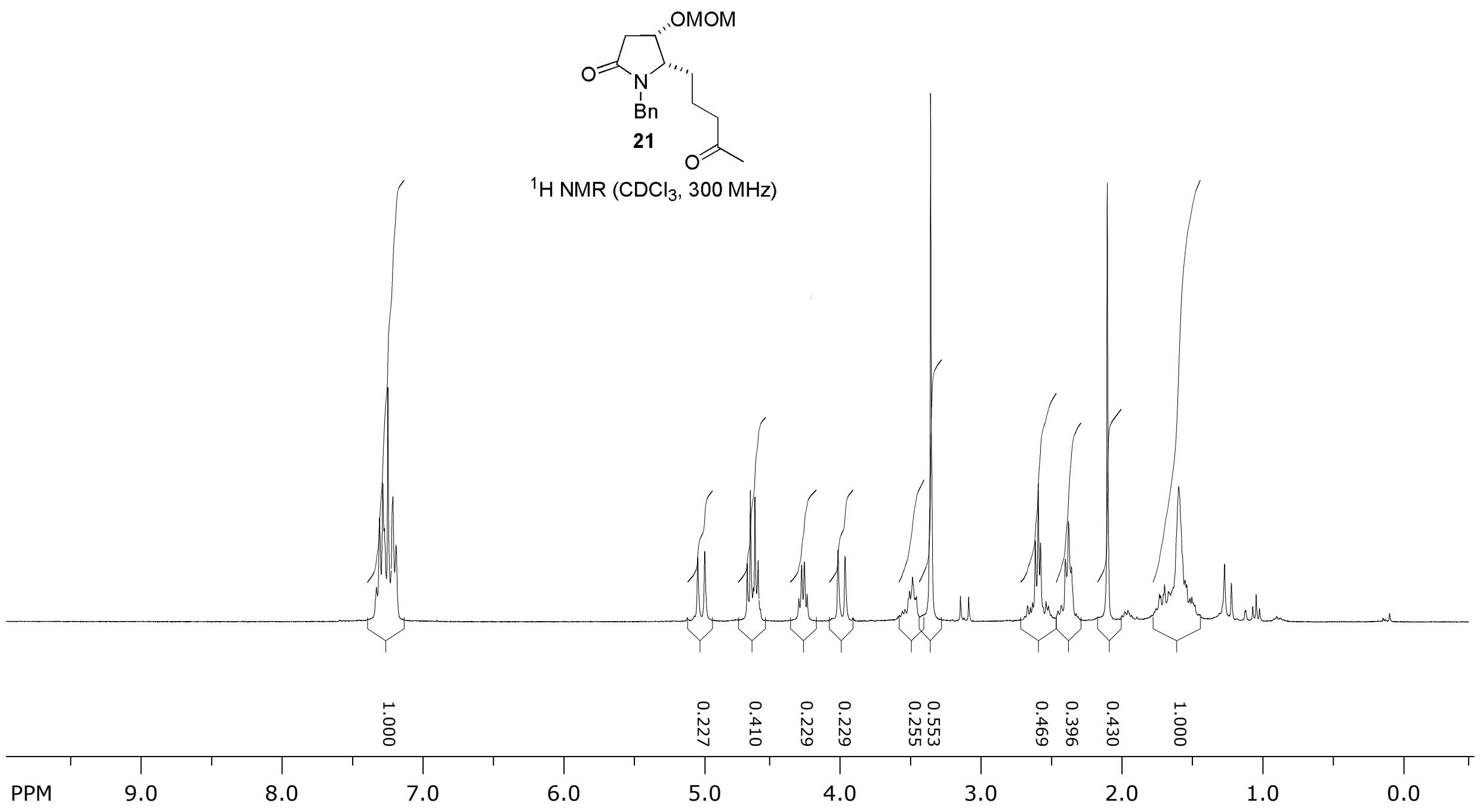

S63 

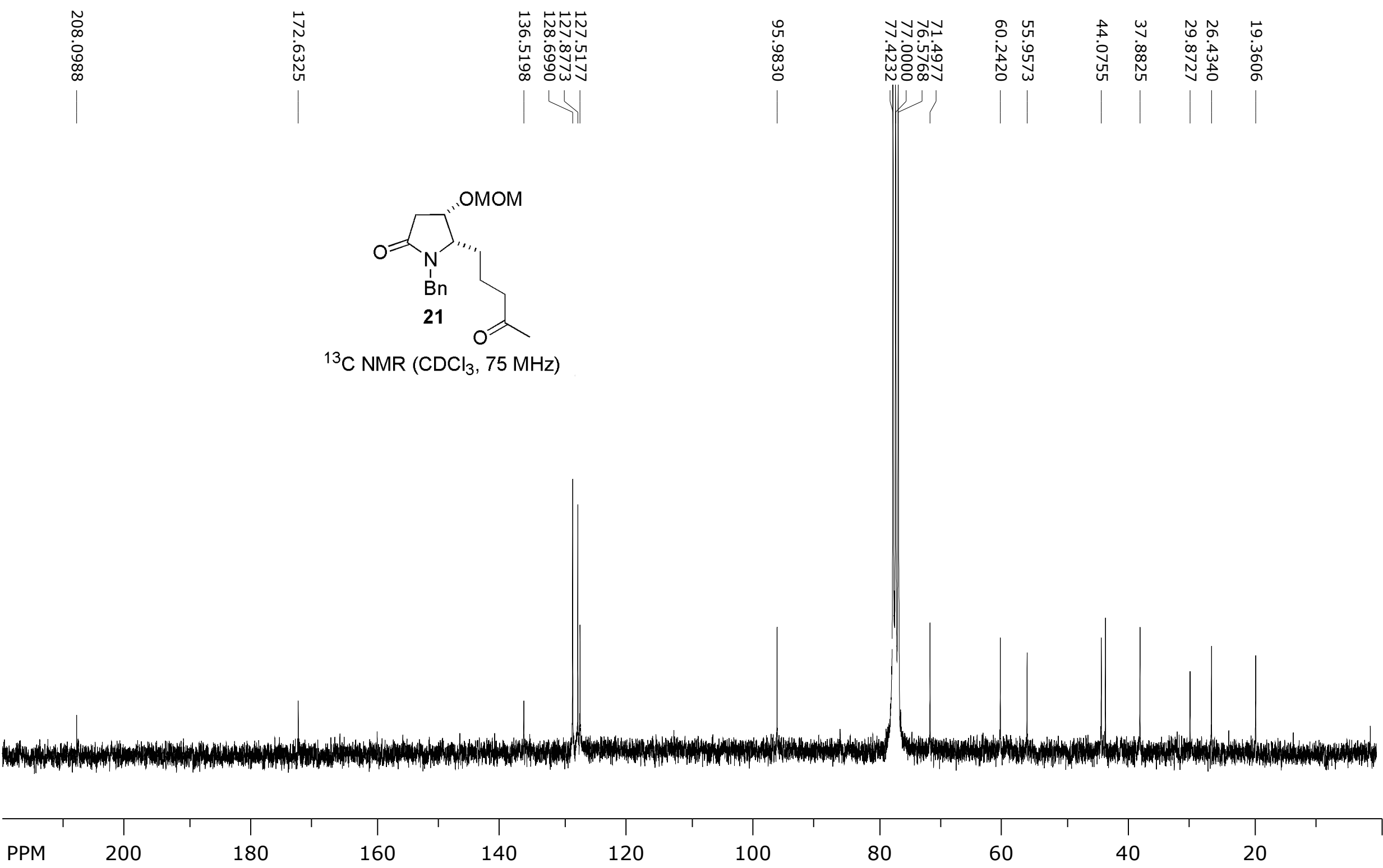


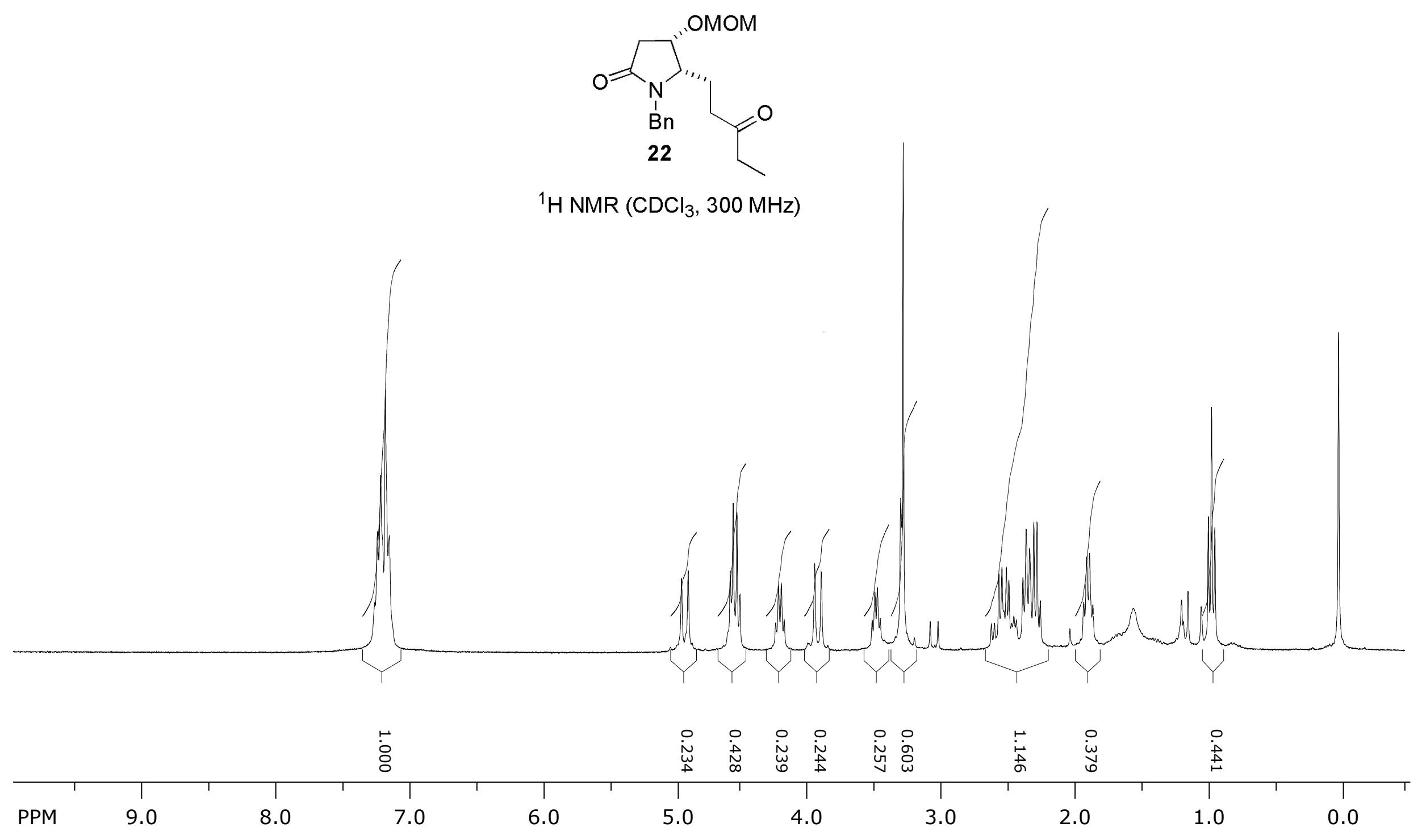



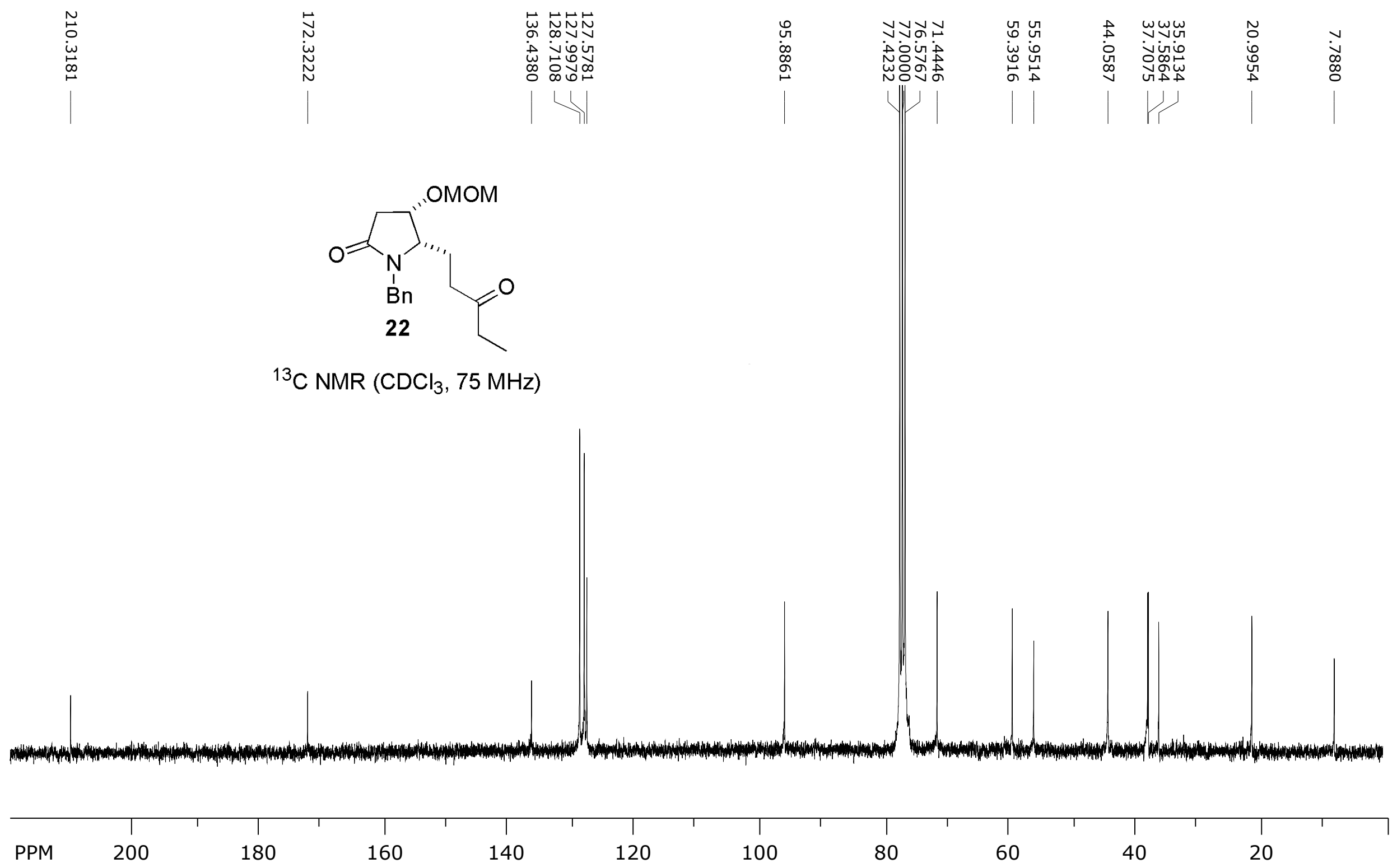


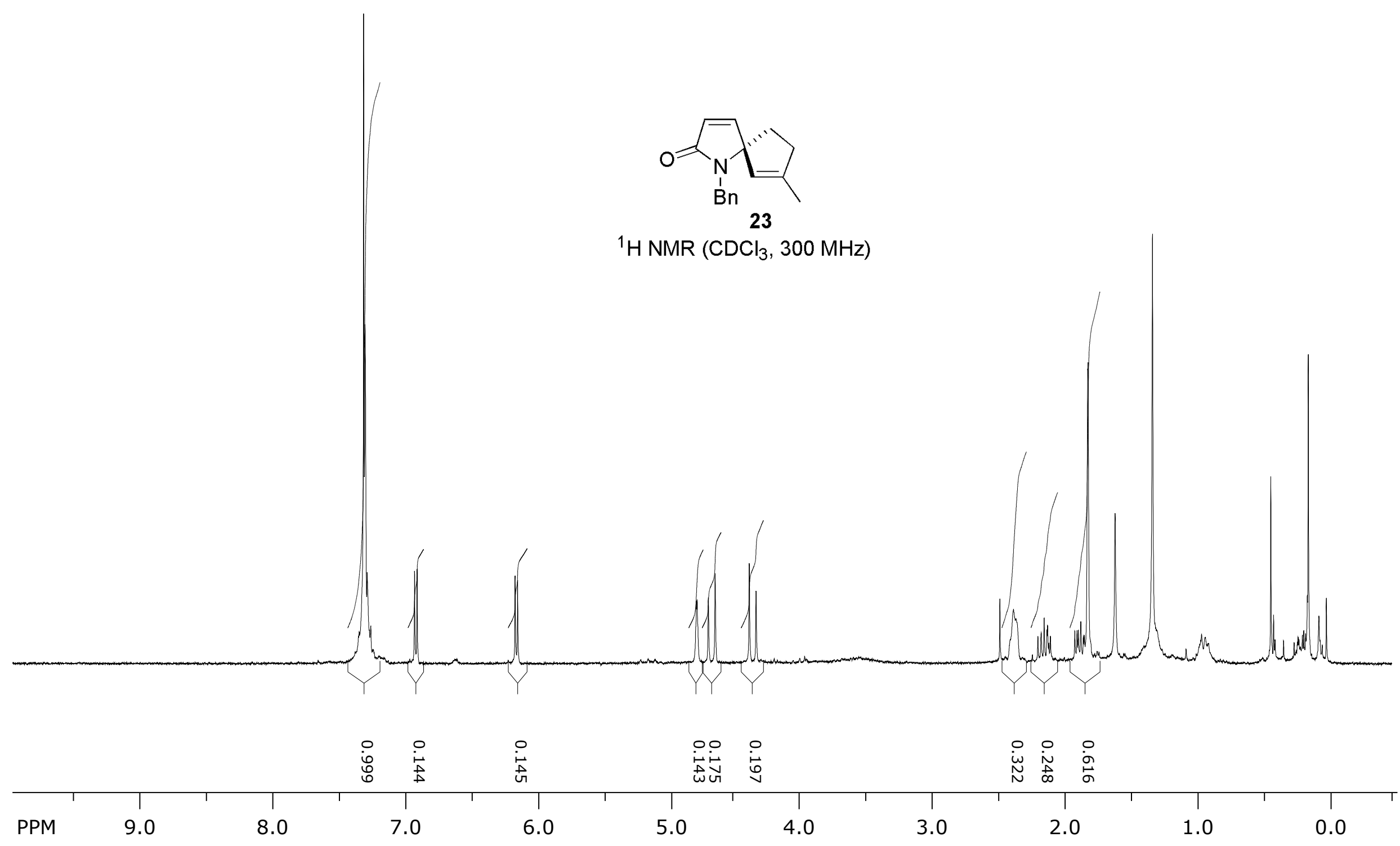




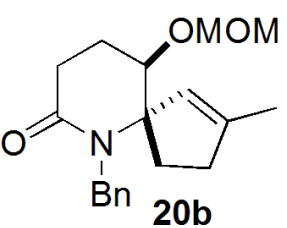

${ }^{1} \mathrm{H} \mathrm{NMR}\left(\mathrm{CDCl}_{3}, 300 \mathrm{MHz}\right)$

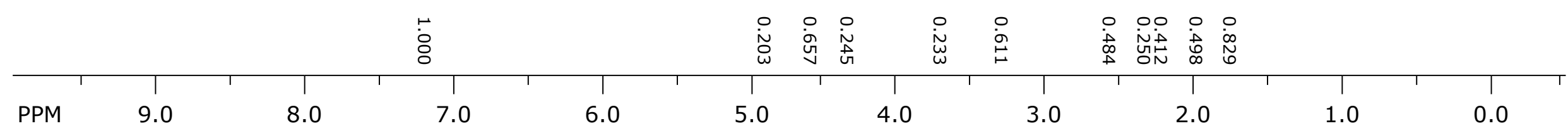




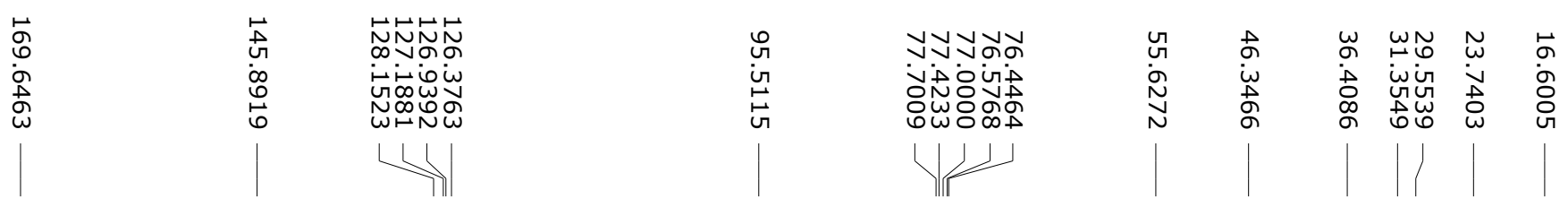

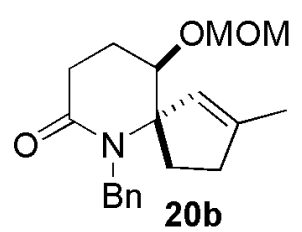

${ }^{13} \mathrm{C} \mathrm{NMR}\left(\mathrm{CDCl}_{3}, 75 \mathrm{MHz}\right)$

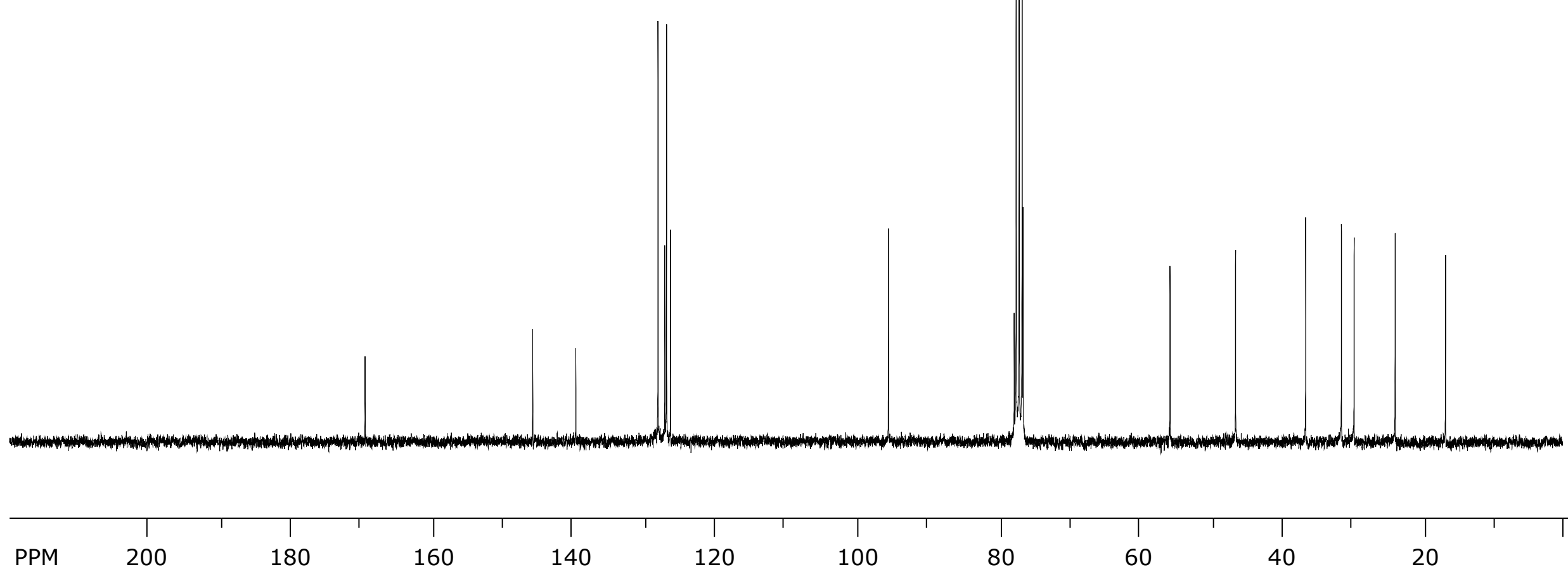




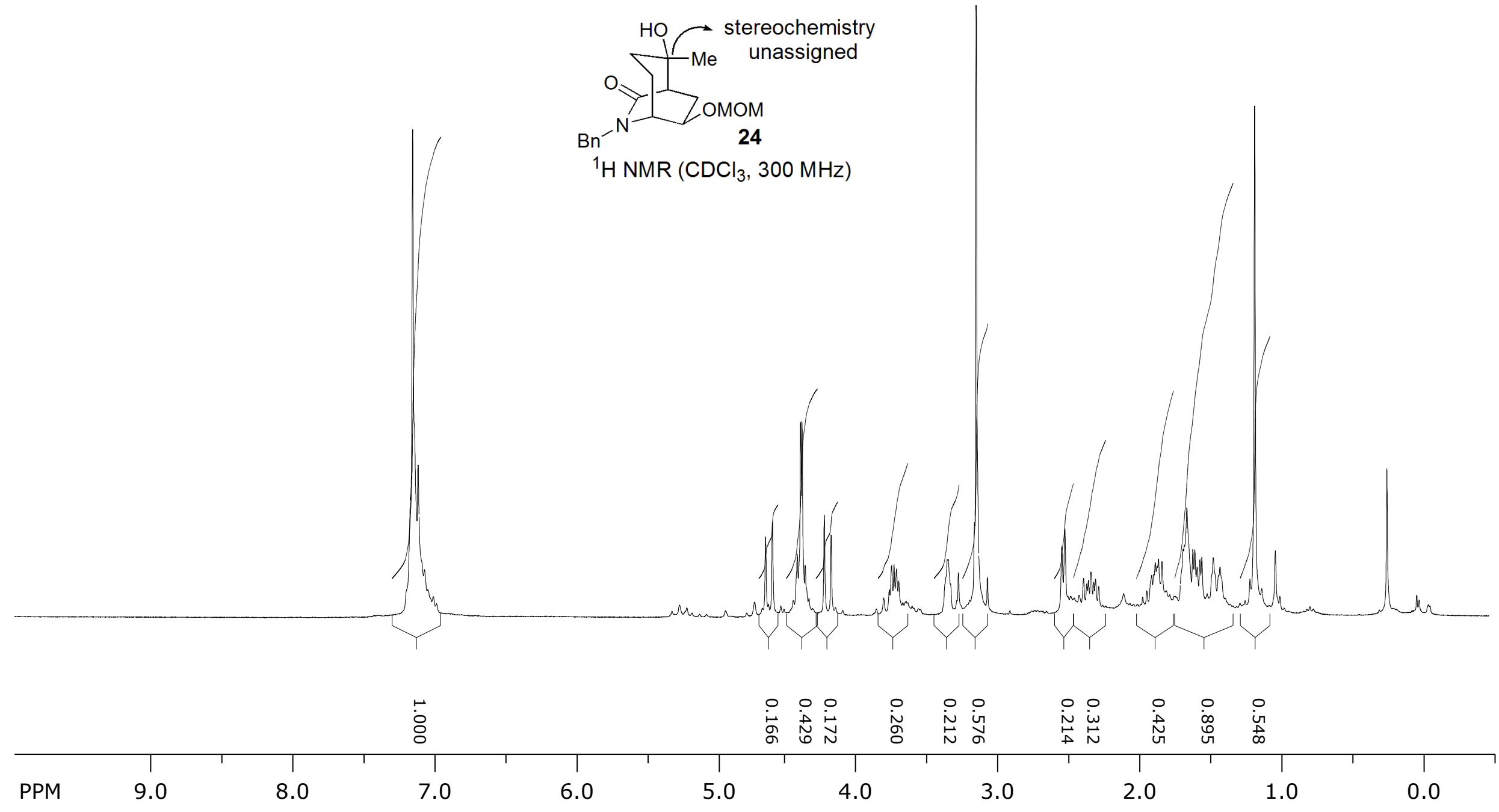



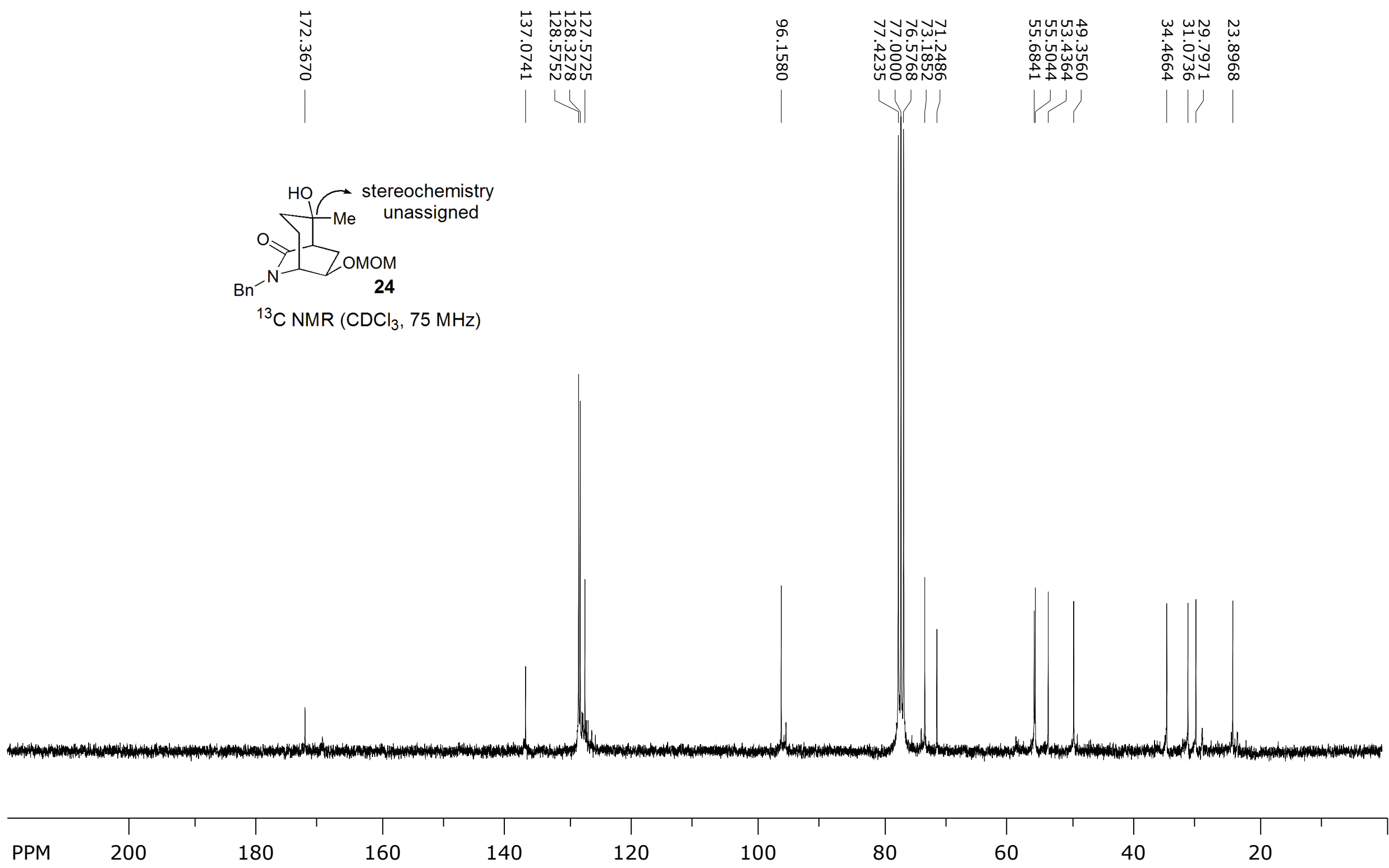


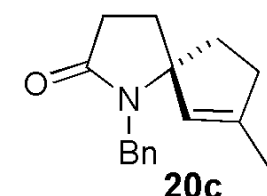

${ }^{1} \mathrm{H}$ NMR $\left(\mathrm{CDCl}_{3}, 300 \mathrm{MHz}\right)$

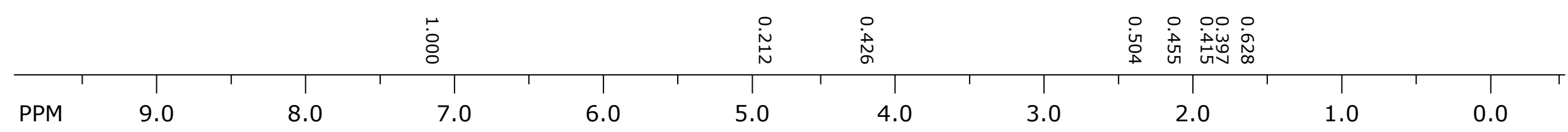




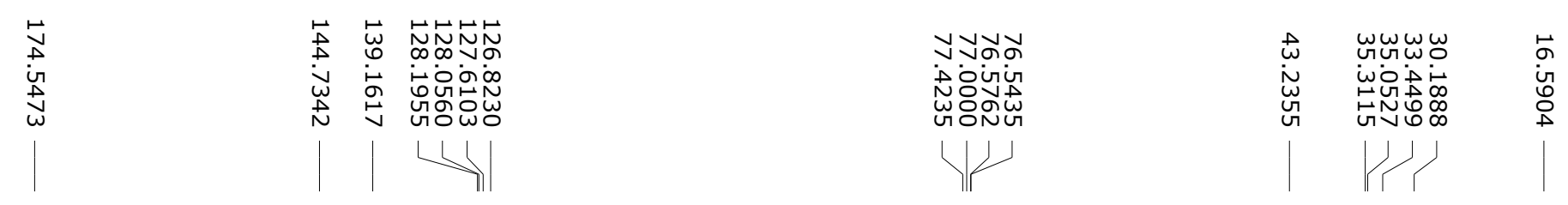

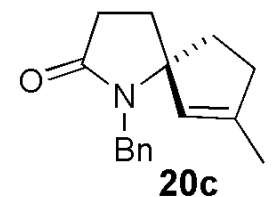

${ }^{13} \mathrm{C} \mathrm{NMR}\left(\mathrm{CDCl}_{3}, 75 \mathrm{MHz}\right)$
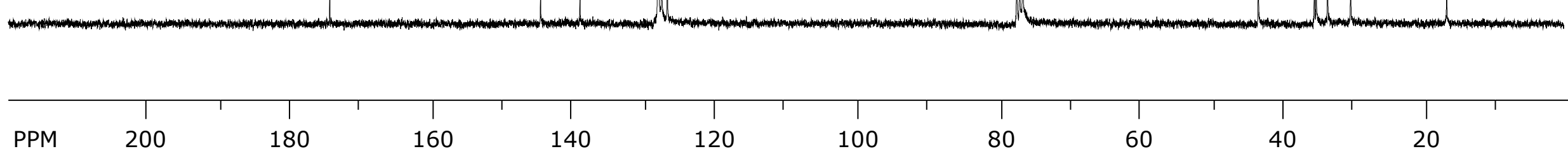


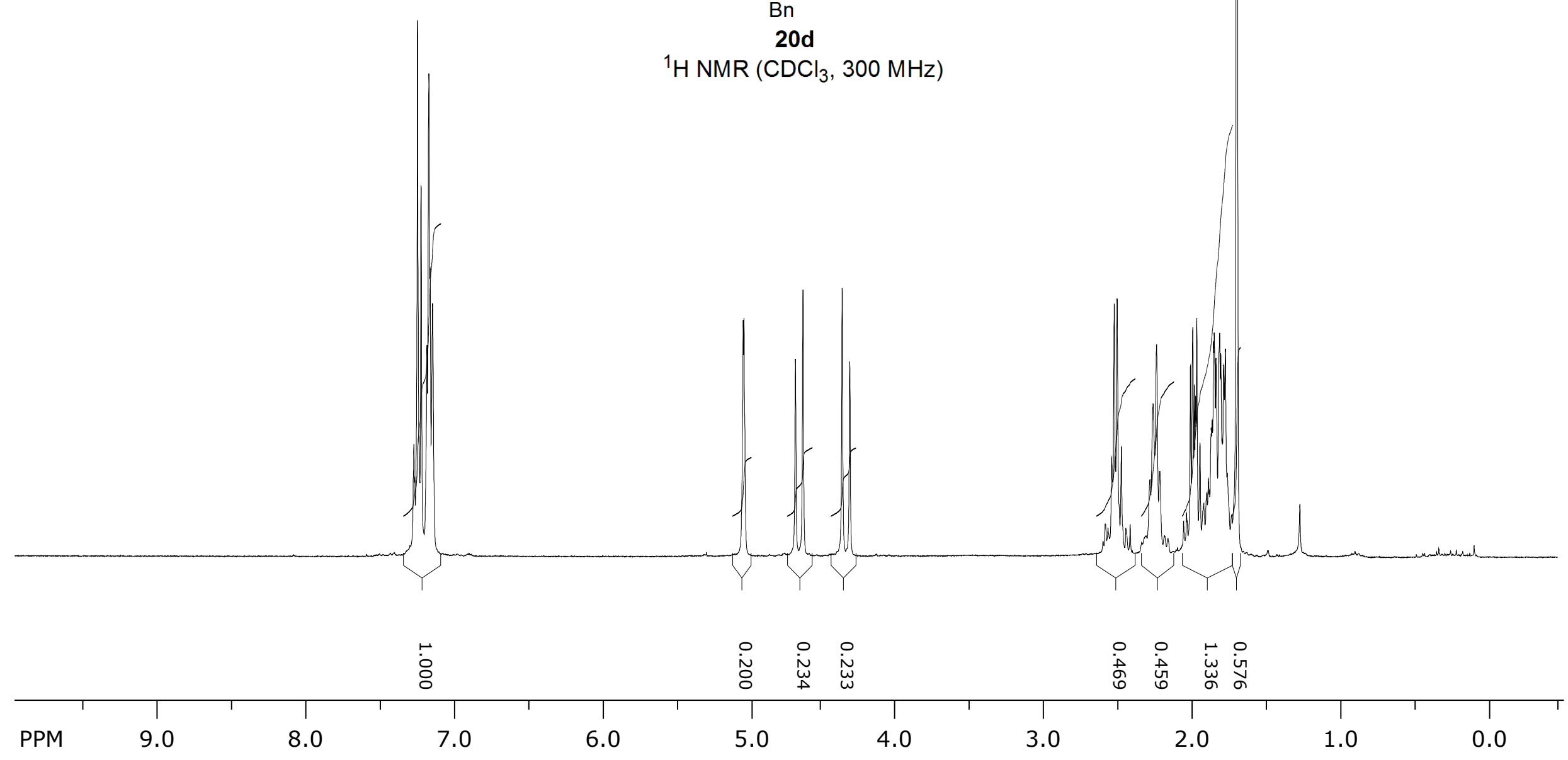




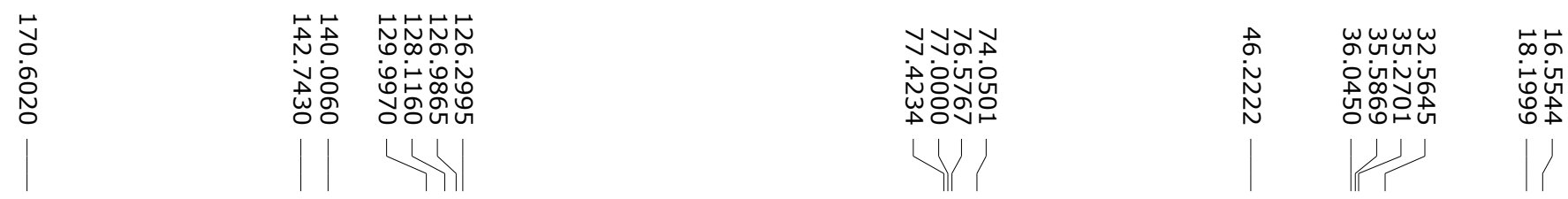

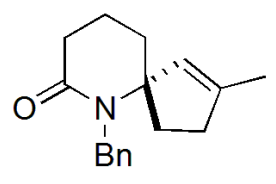

20d

${ }^{13} \mathrm{C} \mathrm{NMR}\left(\mathrm{CDCl}_{3}, 75 \mathrm{MHz}\right)$

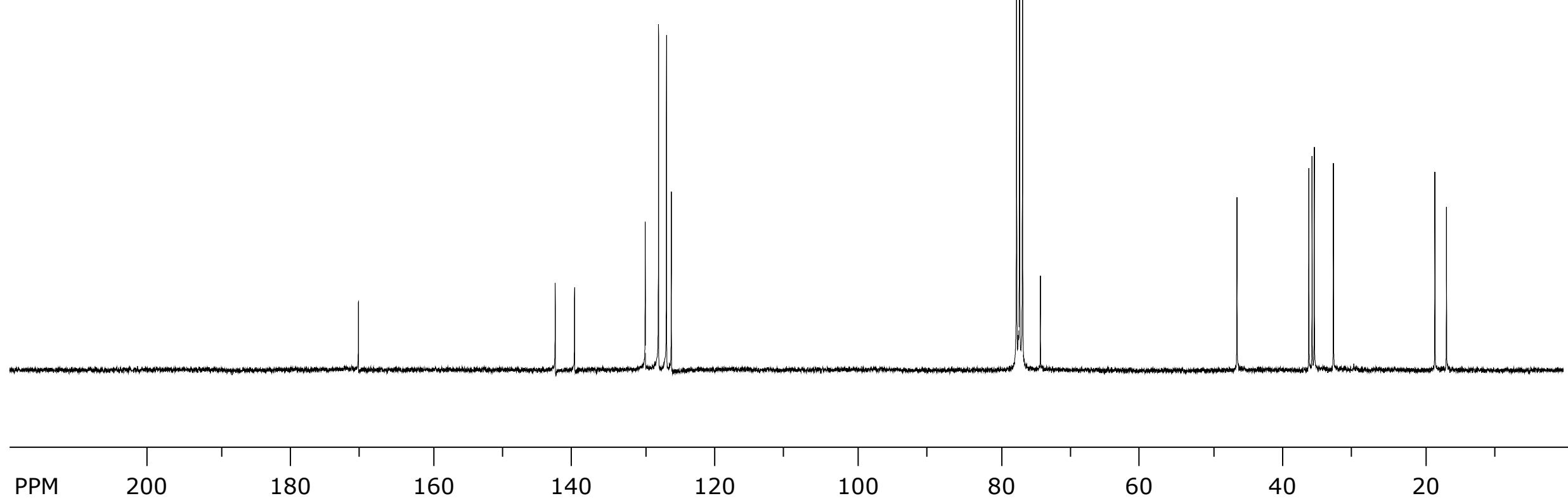




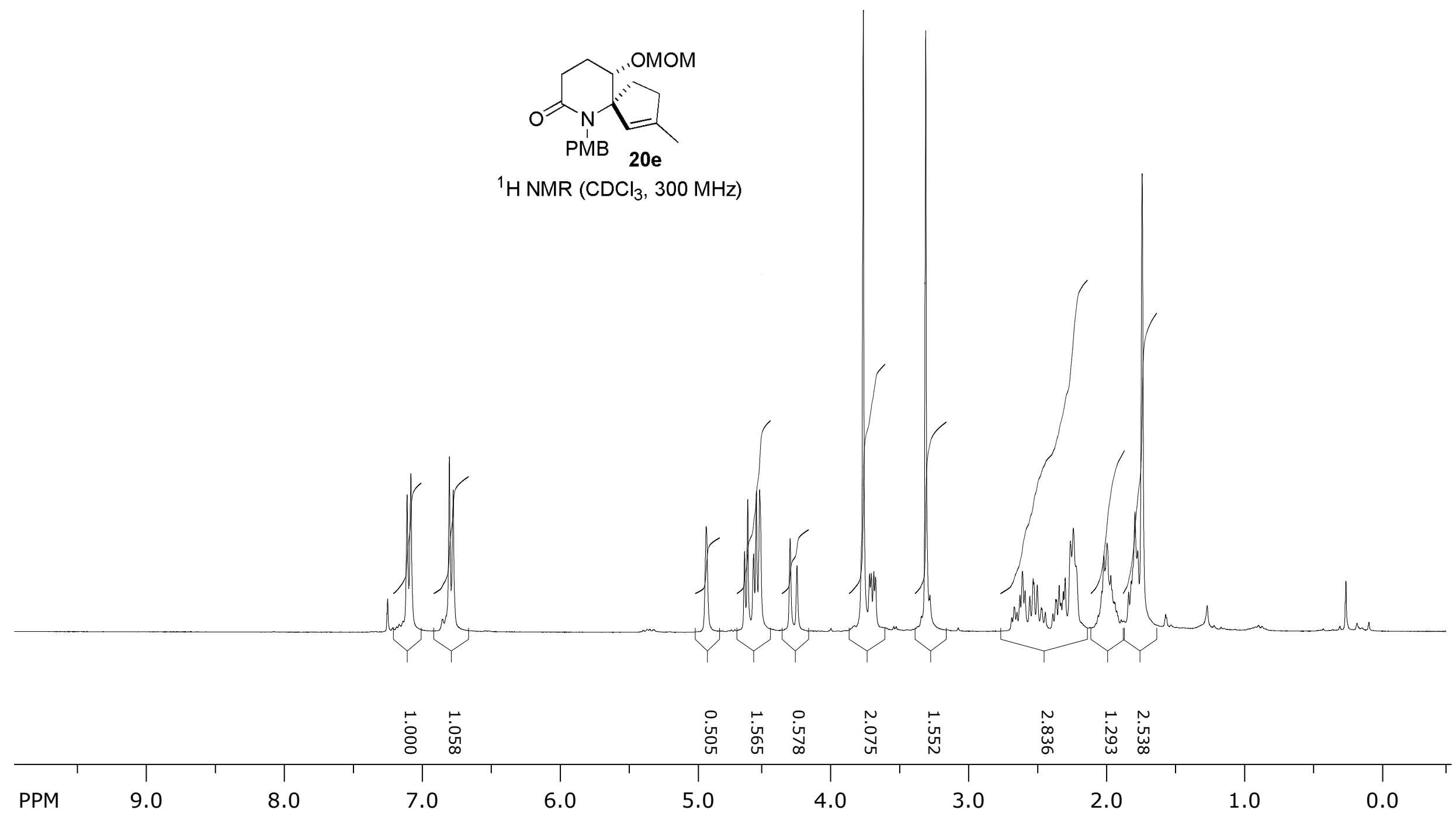




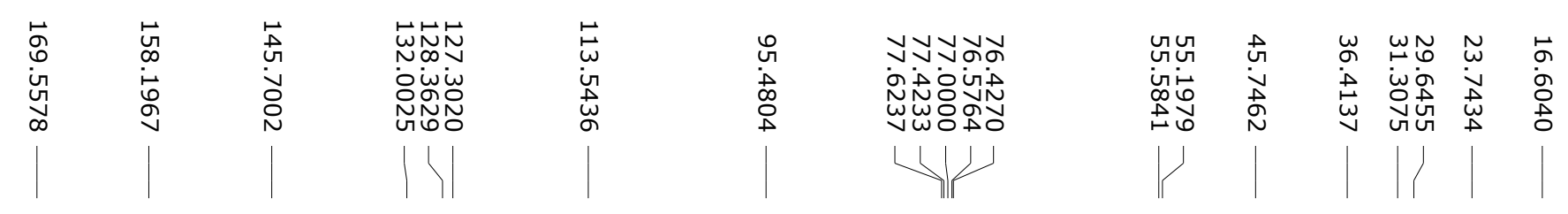

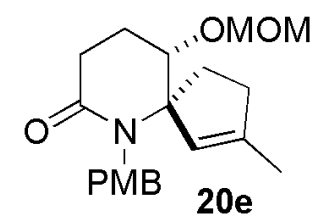

${ }^{13} \mathrm{C} \mathrm{NMR}\left(\mathrm{CDCl}_{3}, 75 \mathrm{MHz}\right)$
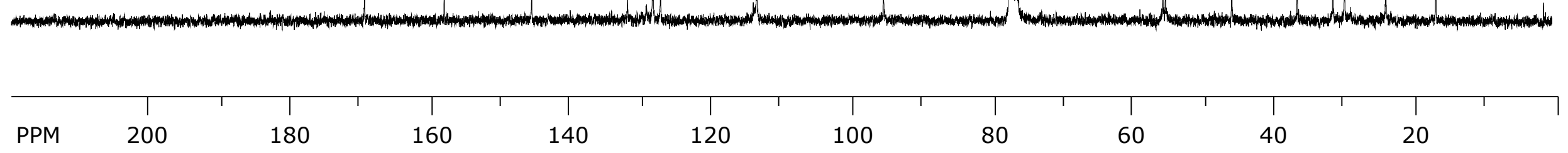


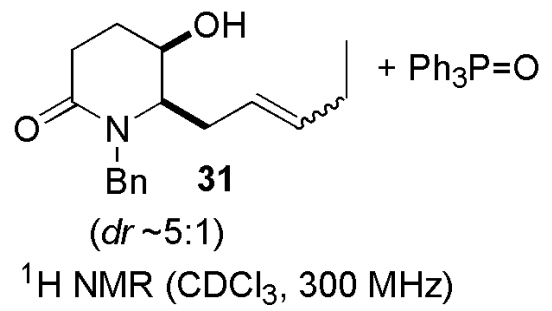

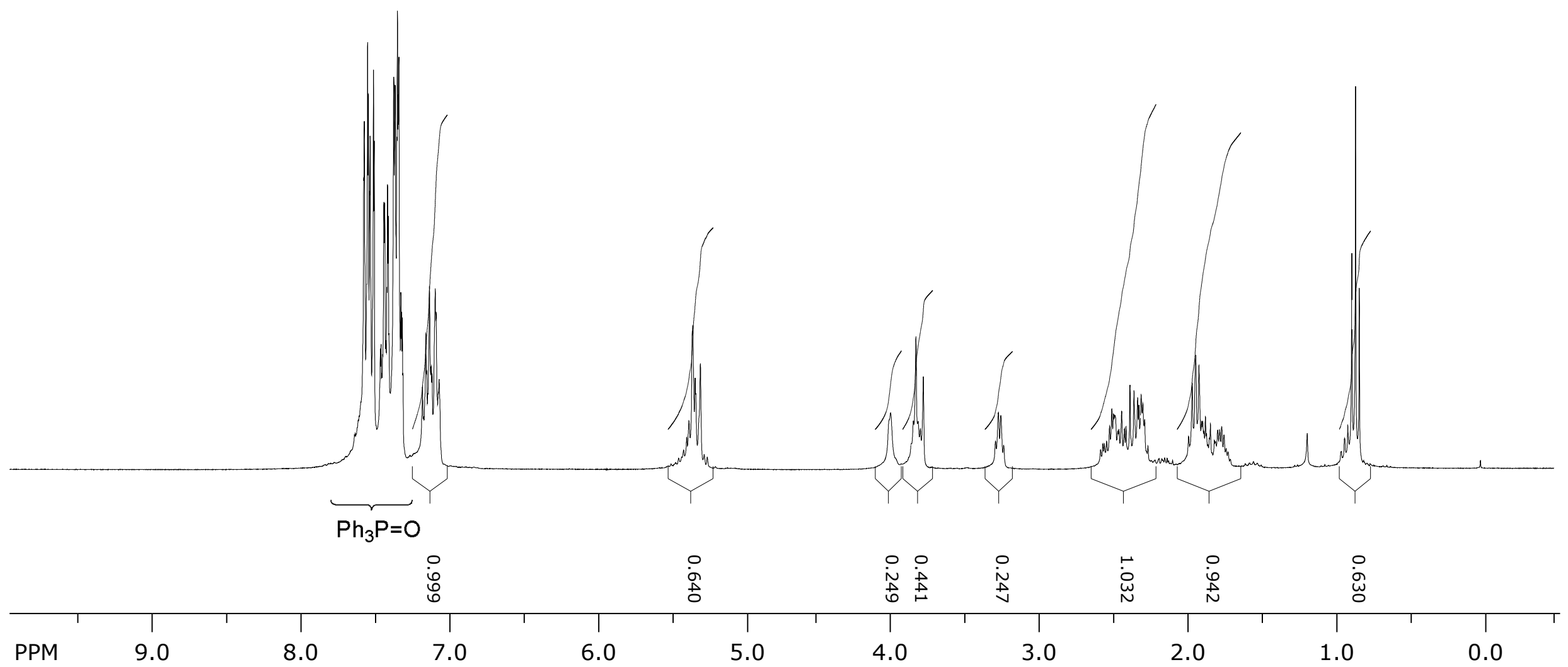

S78 


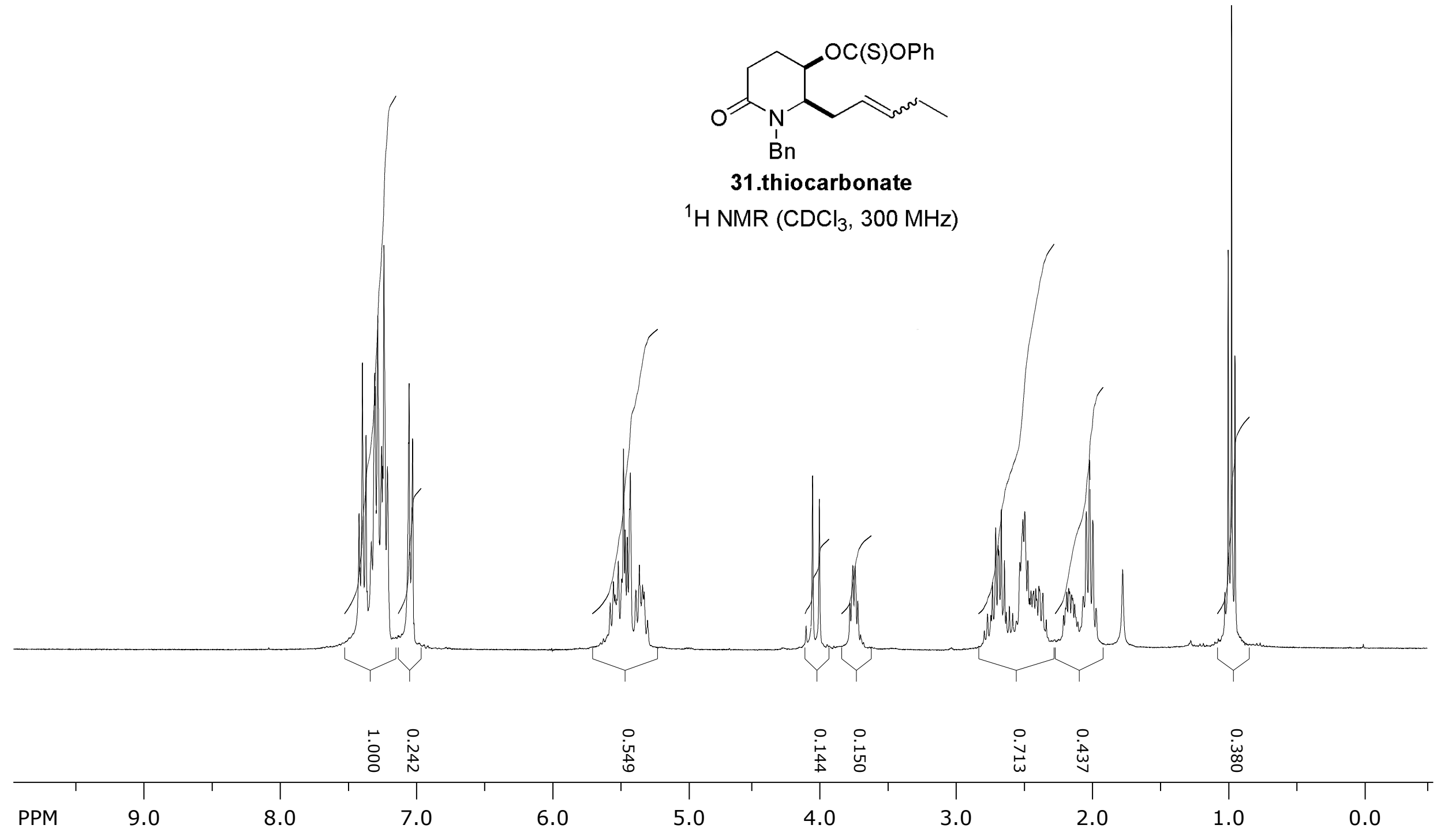




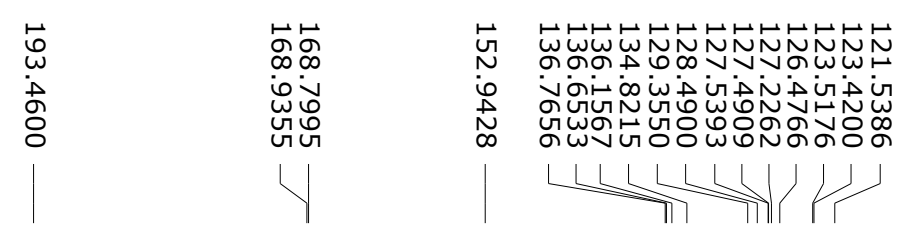

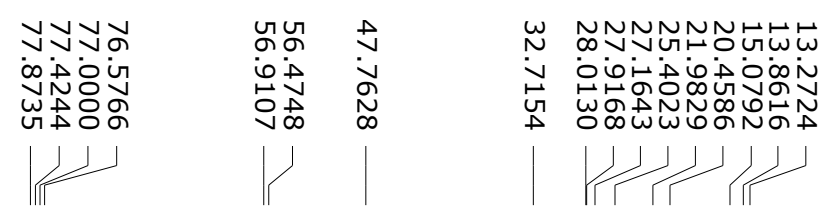

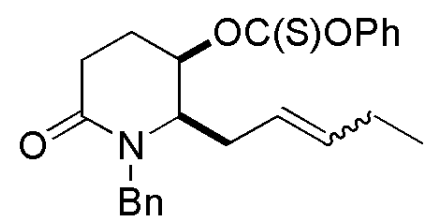

\section{1.thiocarbonate}

${ }^{13} \mathrm{C} \mathrm{NMR}\left(\mathrm{CDCl}_{3}, 75 \mathrm{MHz}\right)$ 


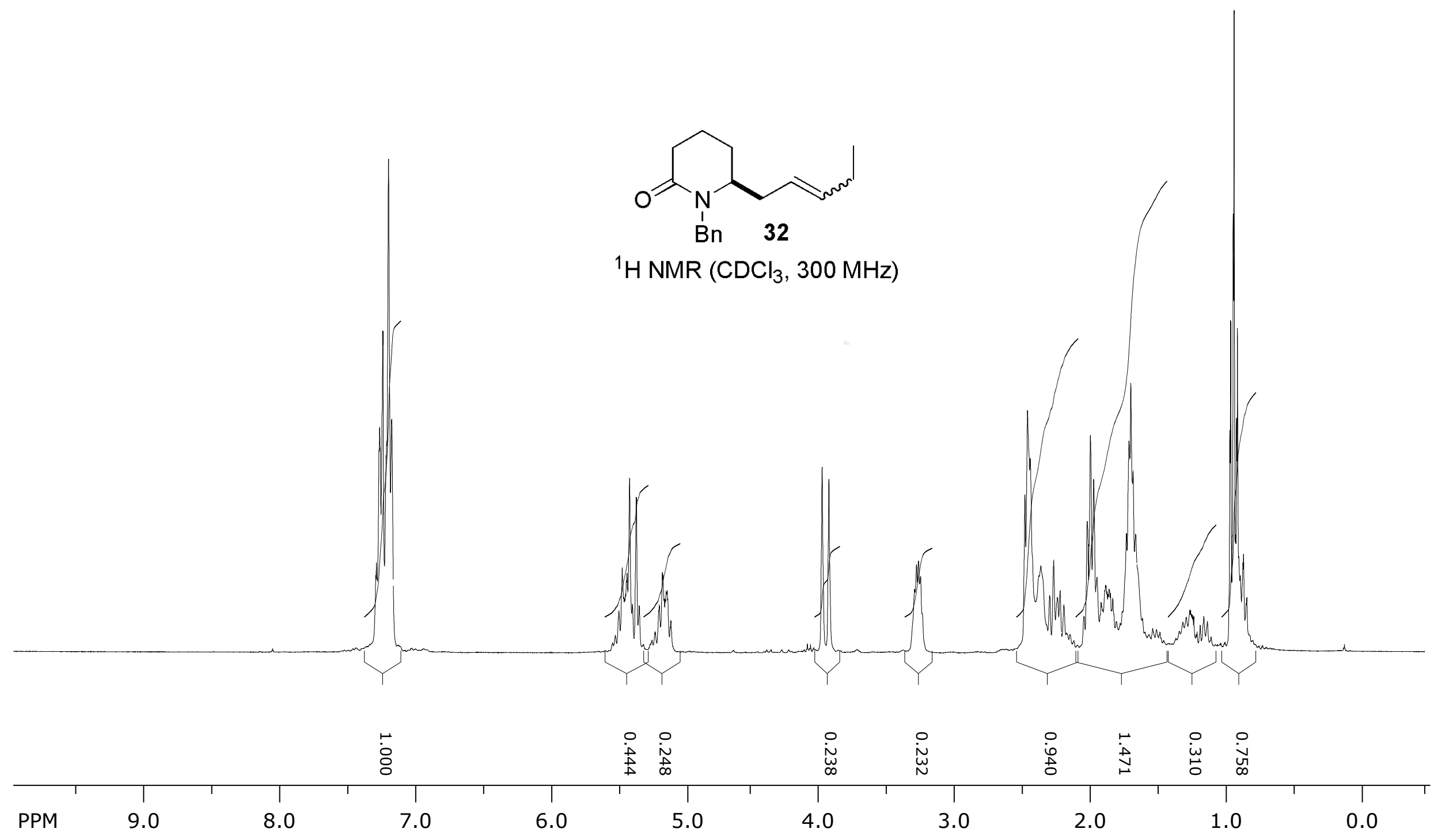



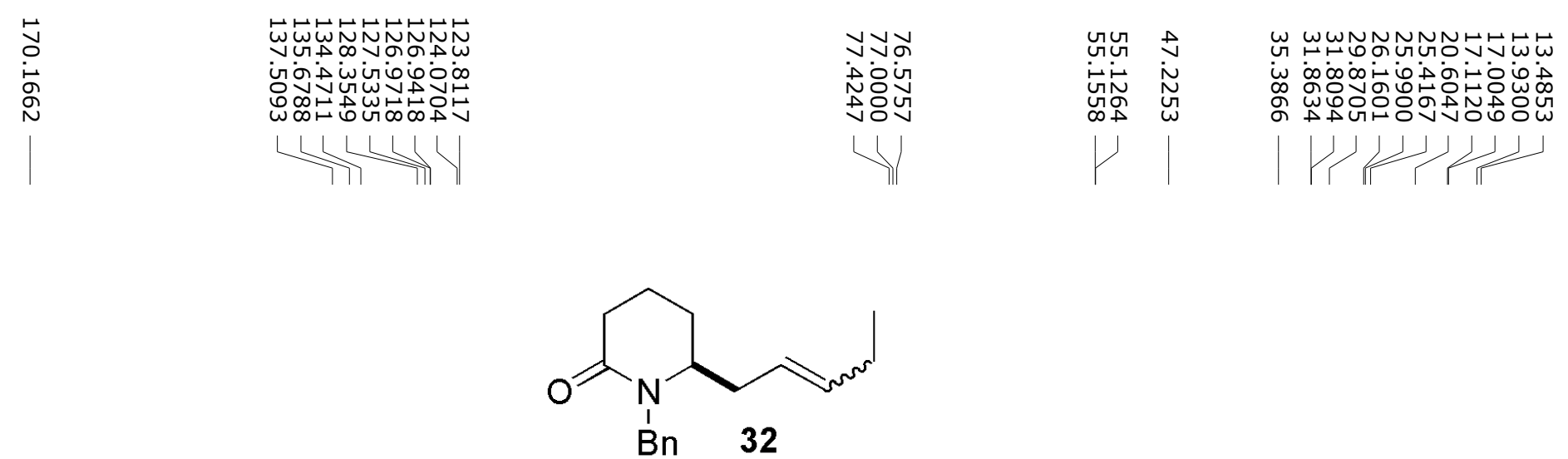

${ }^{13} \mathrm{C} \mathrm{NMR}\left(\mathrm{CDCl}_{3}, 75 \mathrm{MHz}\right)$
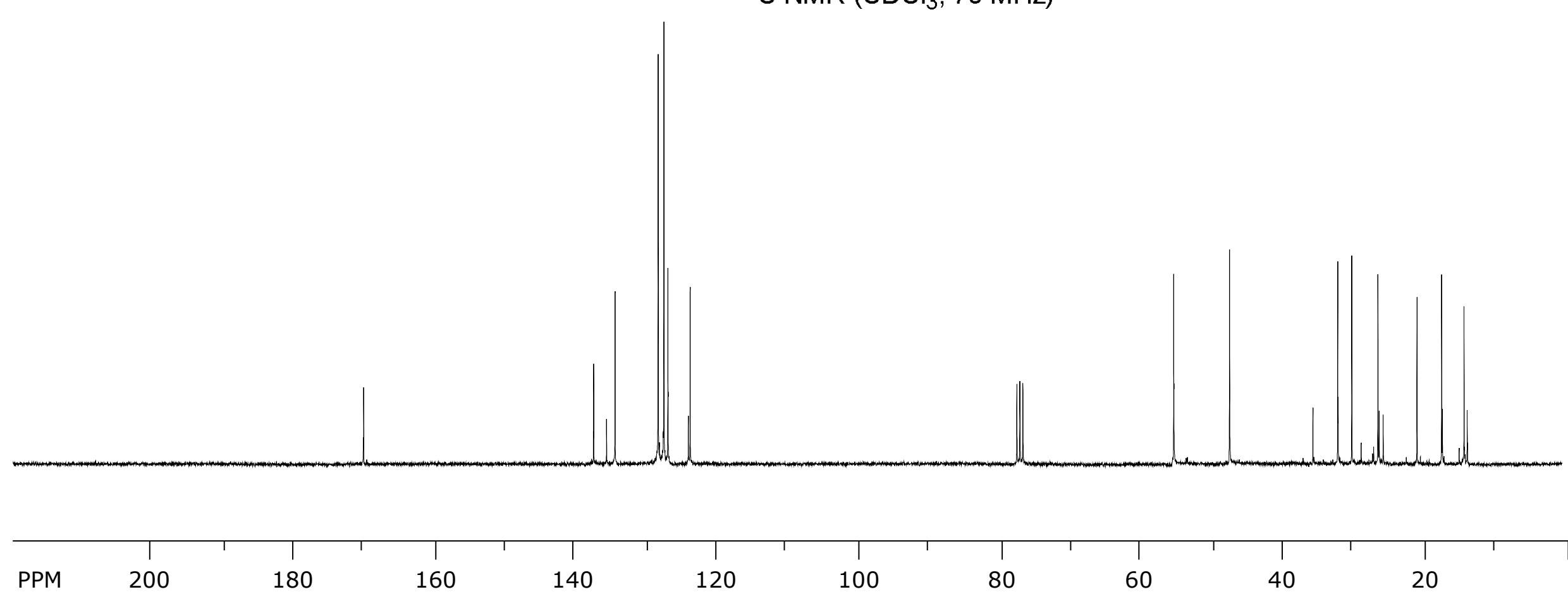


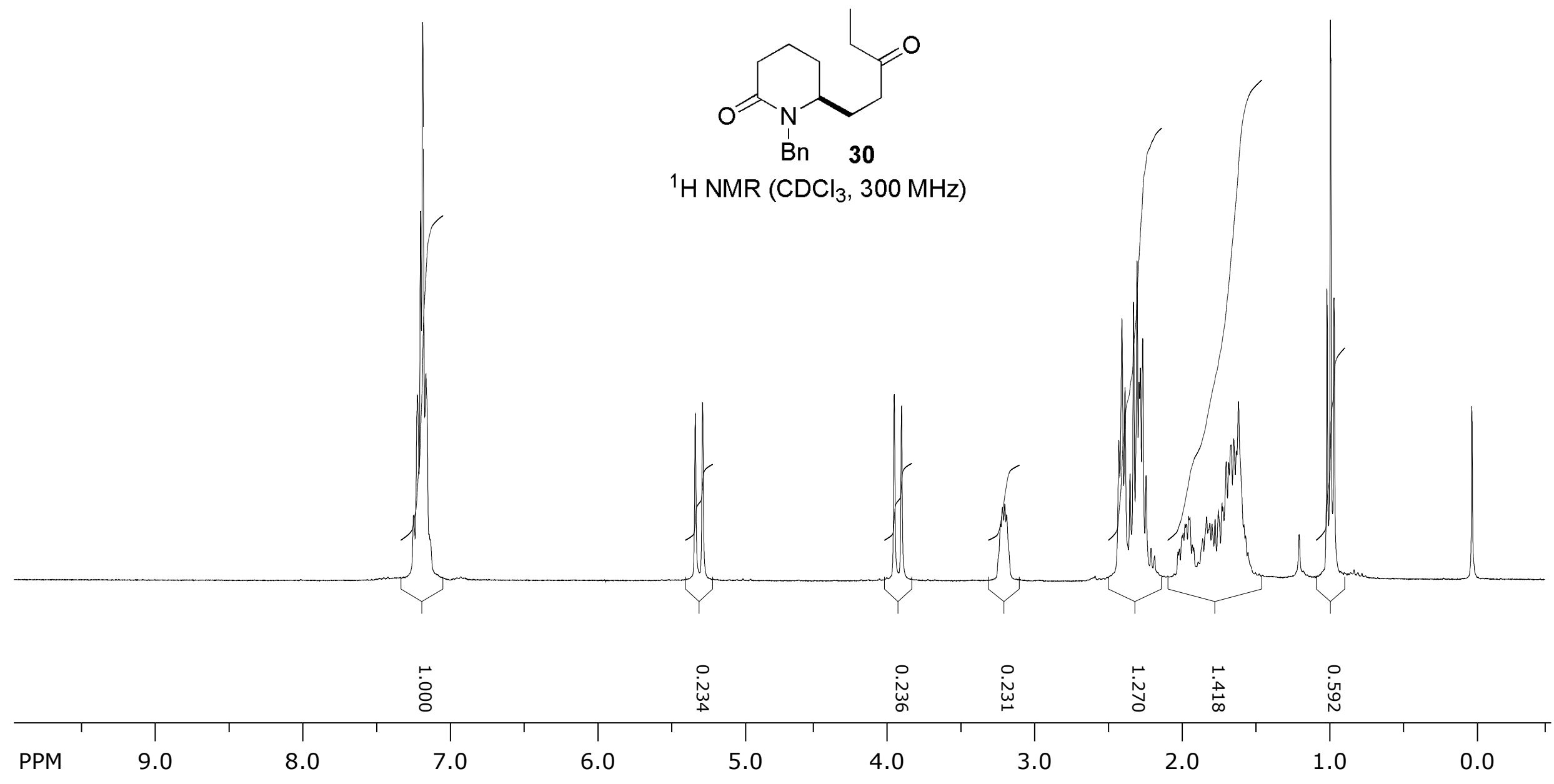




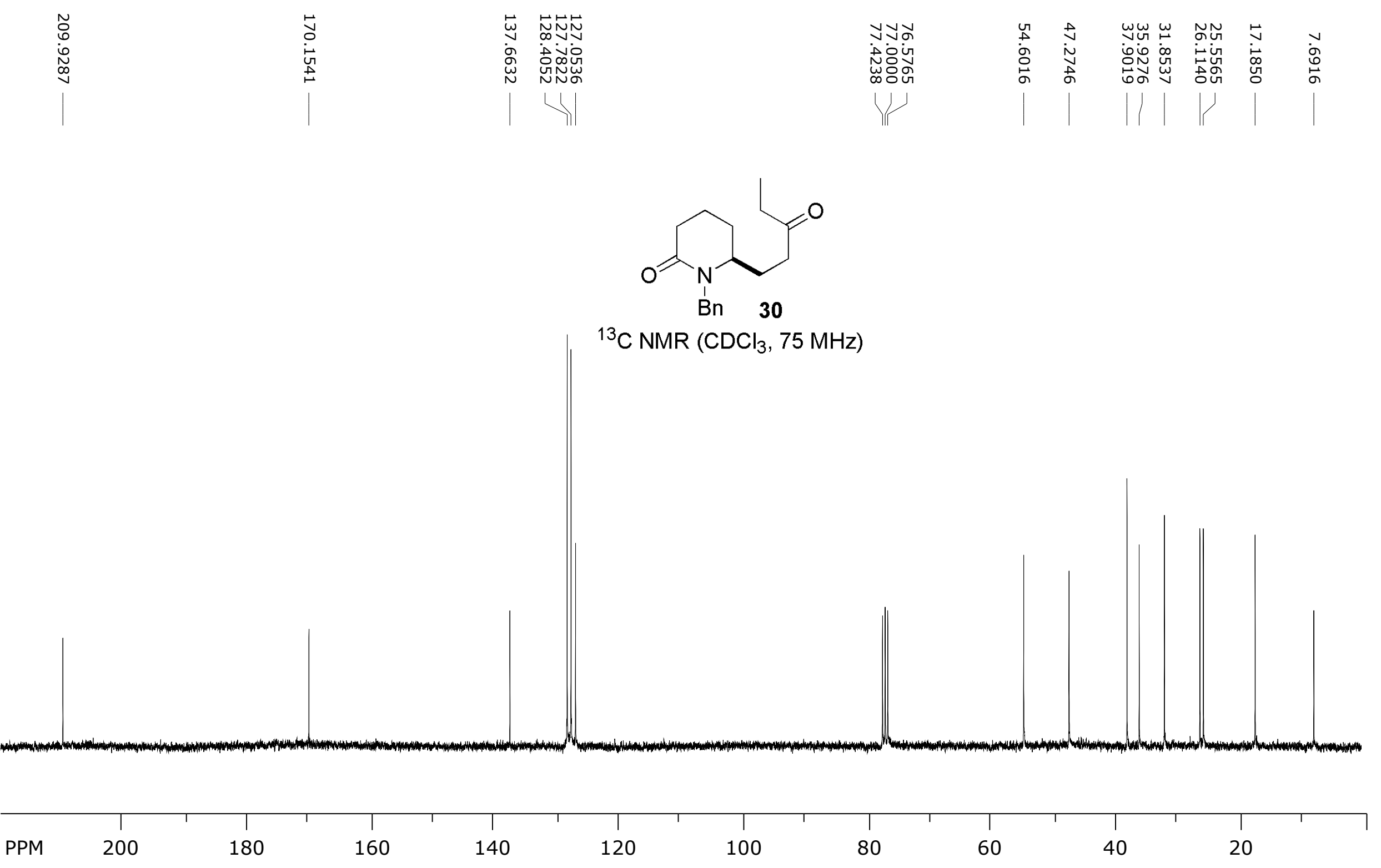




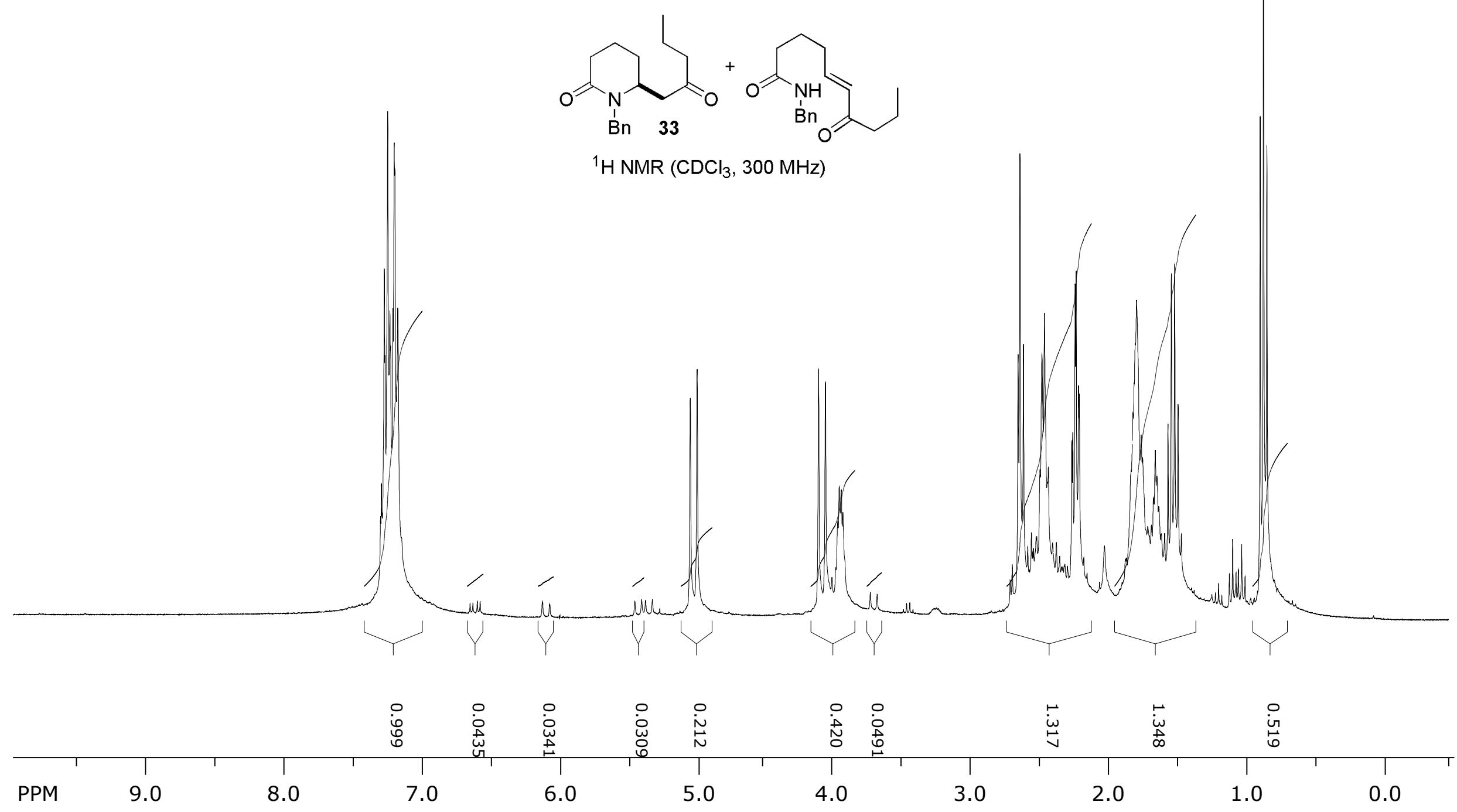




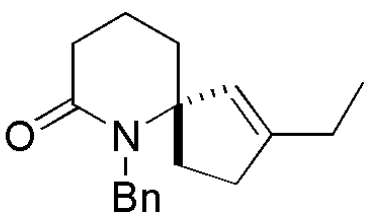

(S)-29

${ }^{1} \mathrm{H} \mathrm{NMR}\left(\mathrm{CDCl}_{3}, 300 \mathrm{MHz}\right)$

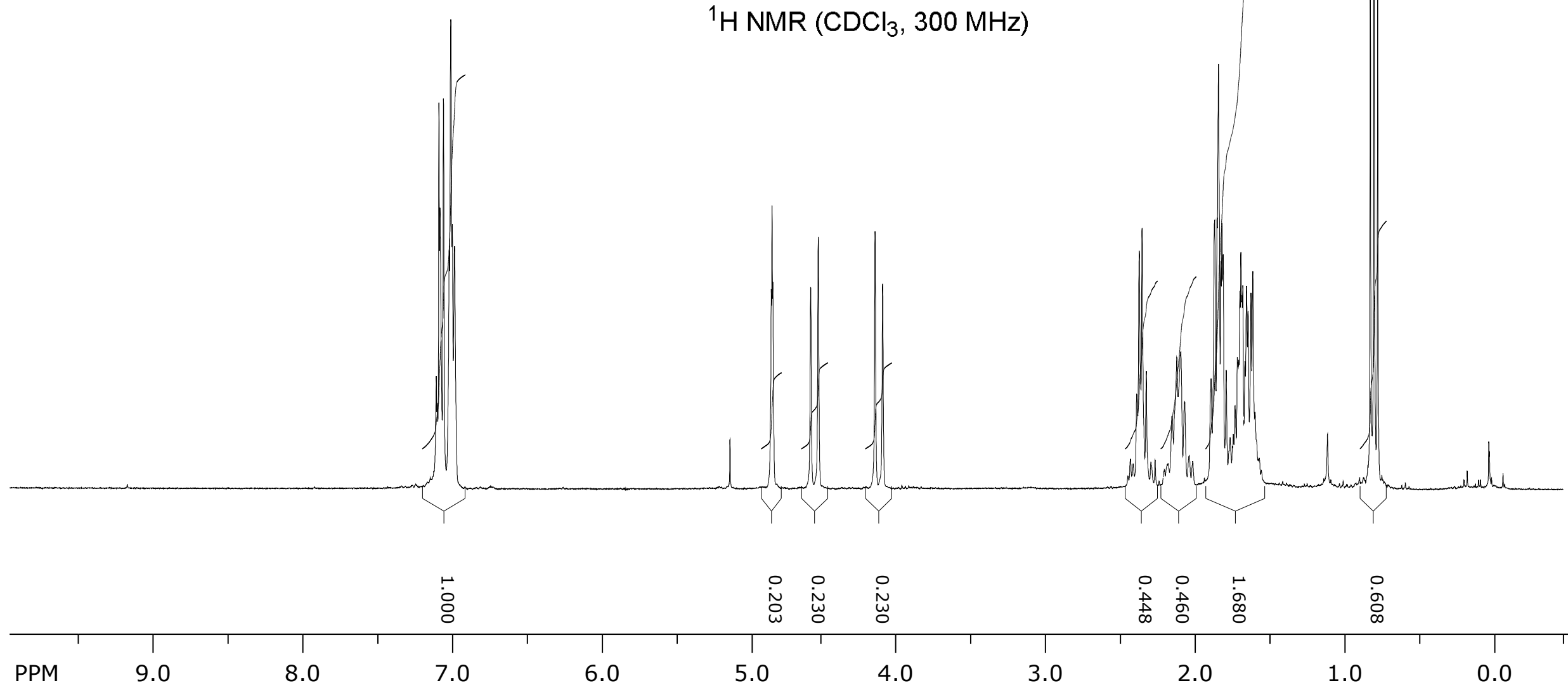




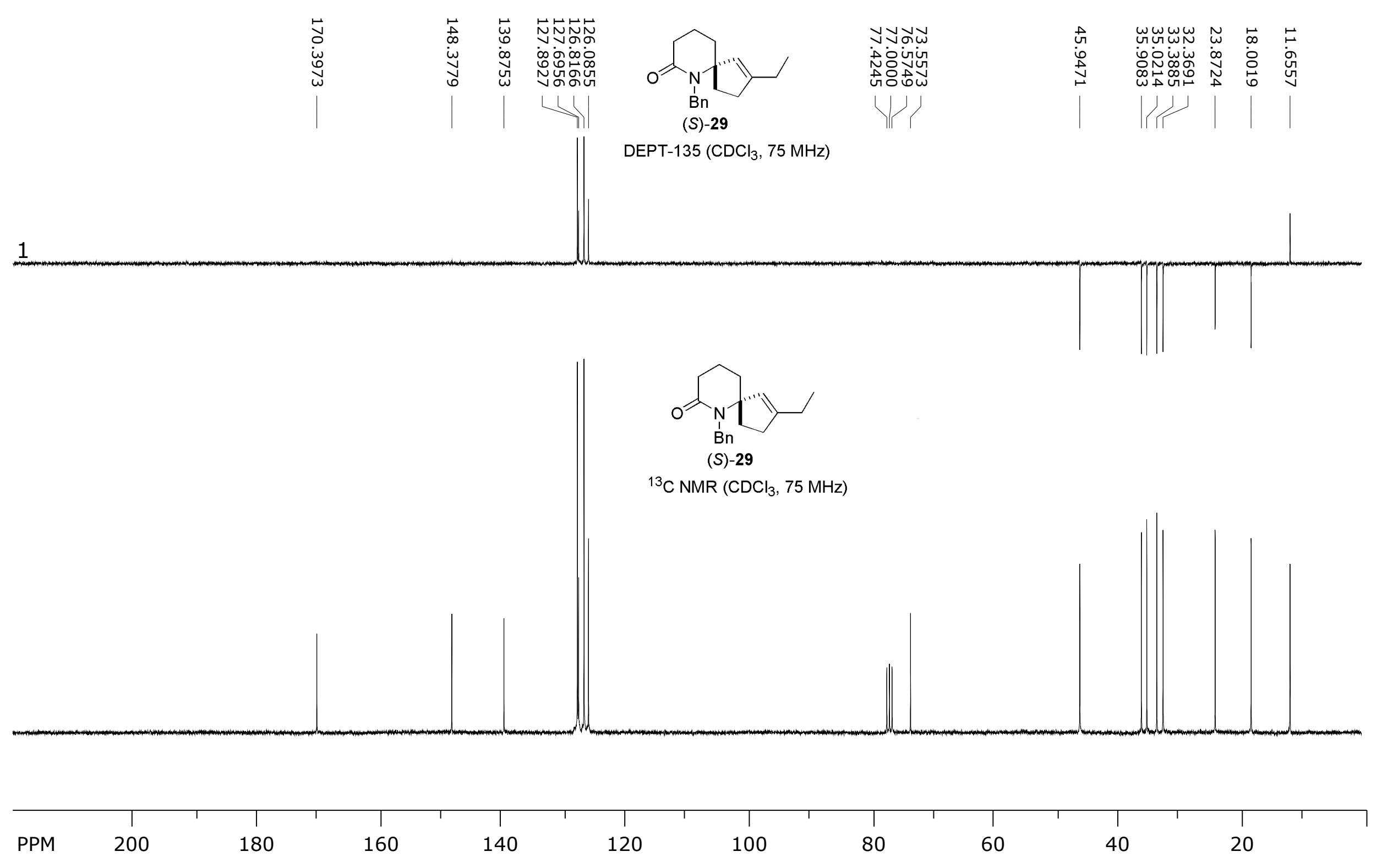




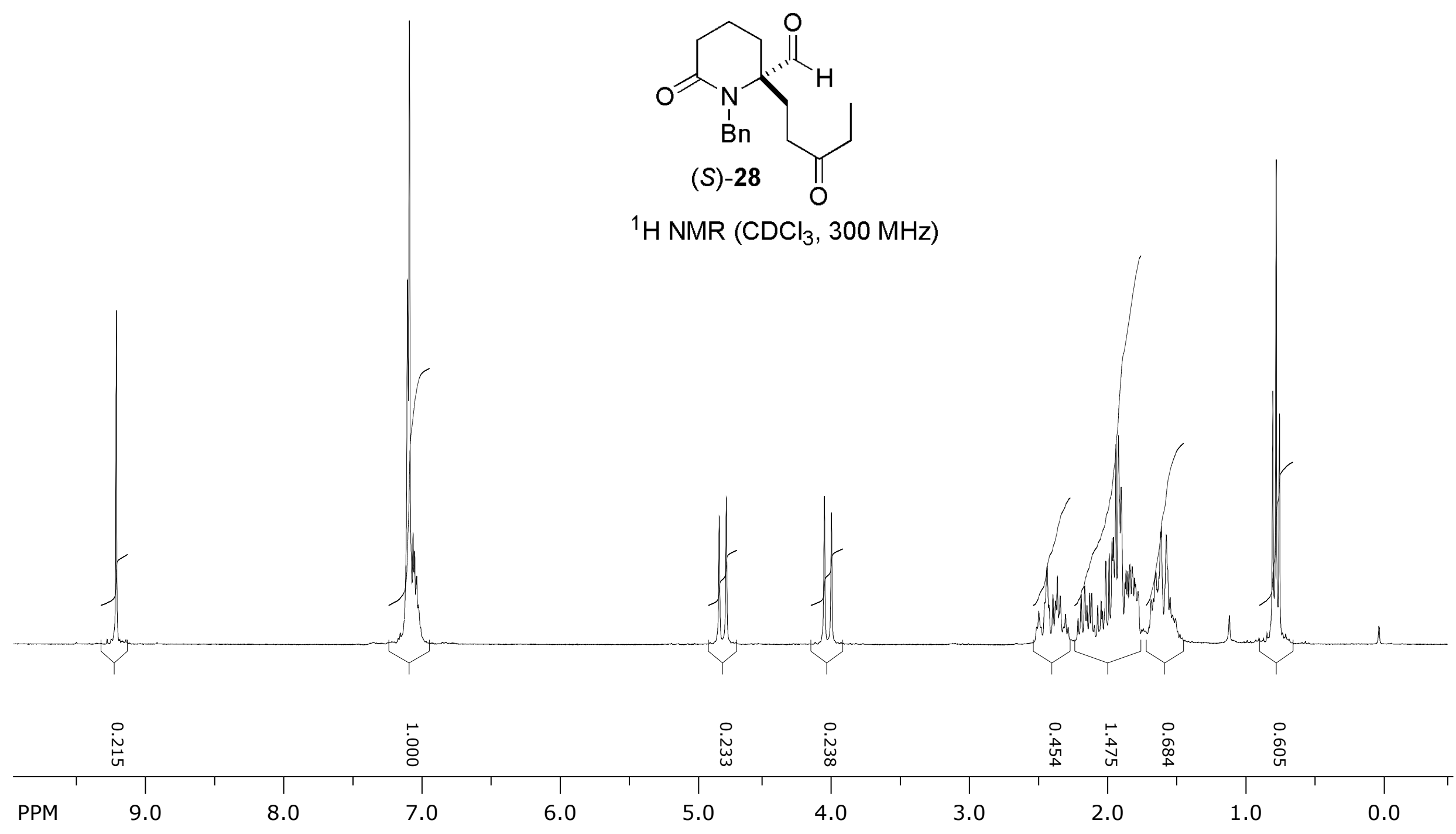



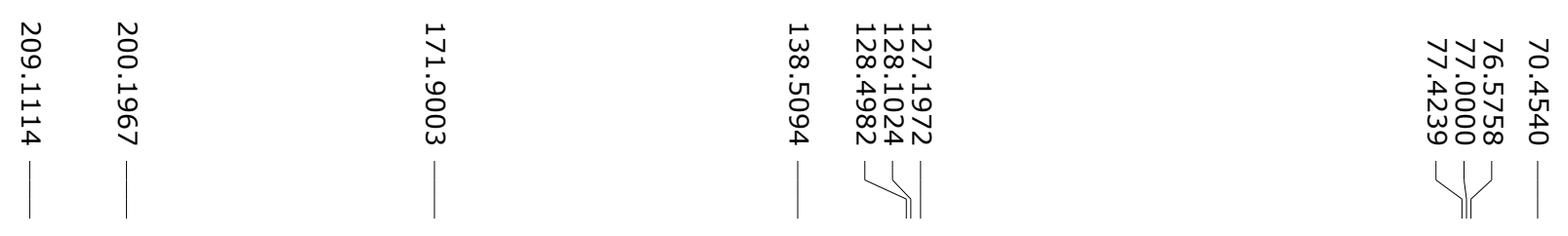

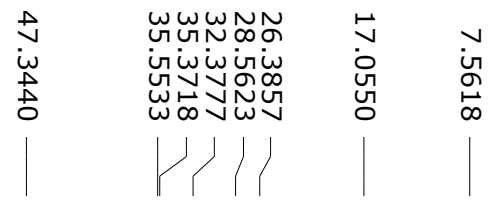

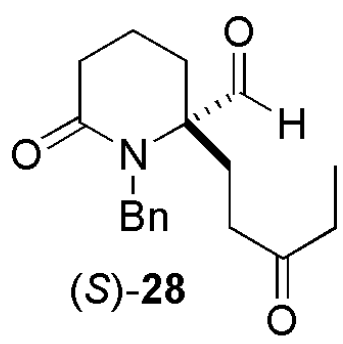

${ }^{13} \mathrm{C} \mathrm{NMR}\left(\mathrm{CDCl}_{3}, 75 \mathrm{MHz}\right)$
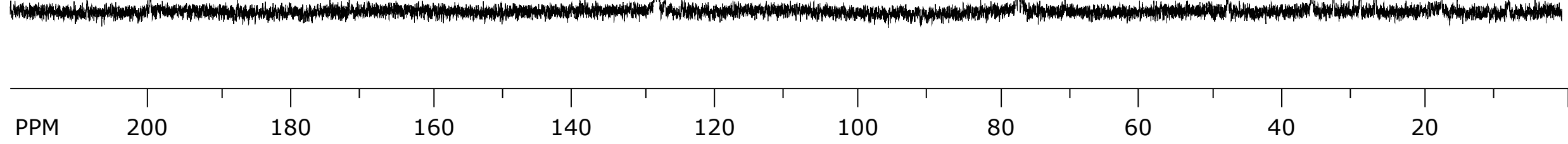


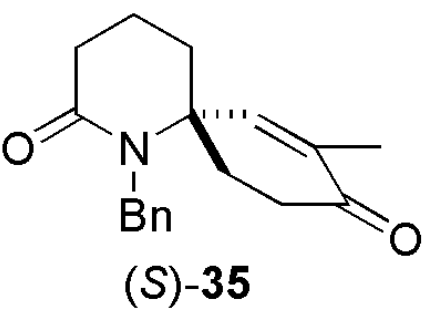

${ }^{1} \mathrm{H}$ NMR $\left(\mathrm{CDCl}_{3}, 300 \mathrm{MHz}\right)$

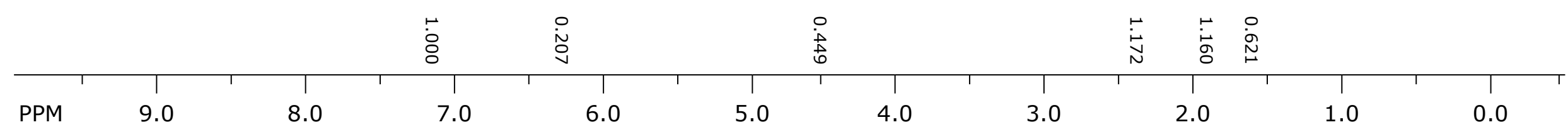




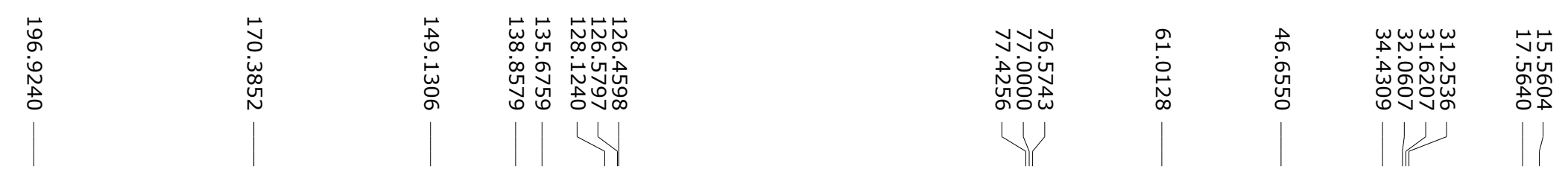

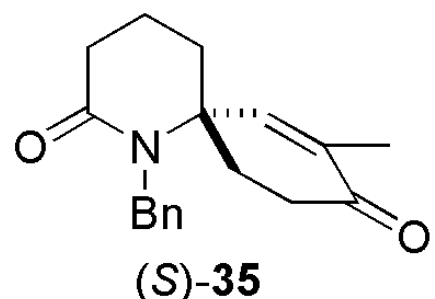

(S) -35

${ }^{13} \mathrm{C} \mathrm{NMR}\left(\mathrm{CDCl}_{3}, 75 \mathrm{MHz}\right)$

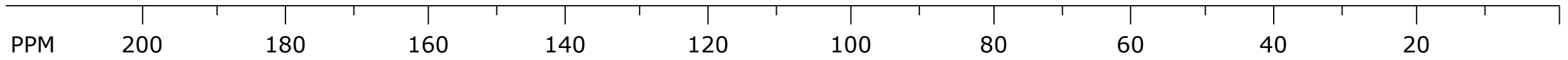




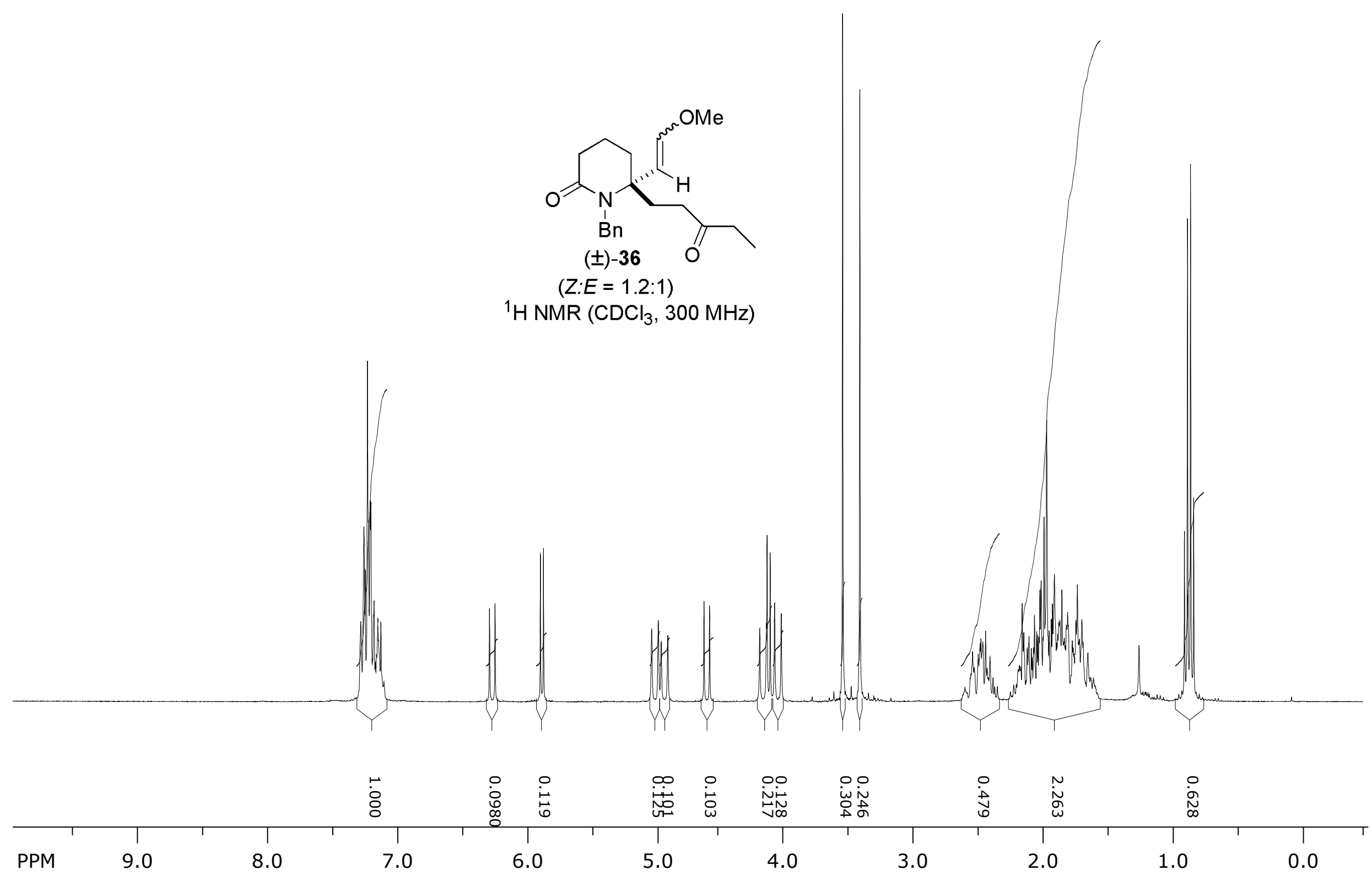




\begin{tabular}{|c|c|c|c|c|c|c|}
\hline 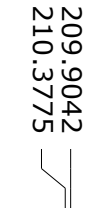 & 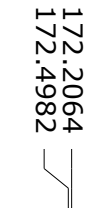 & 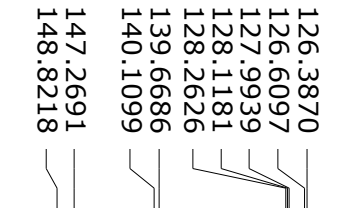 & 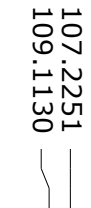 & 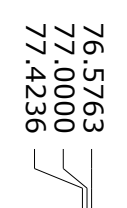 & 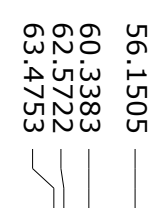 & 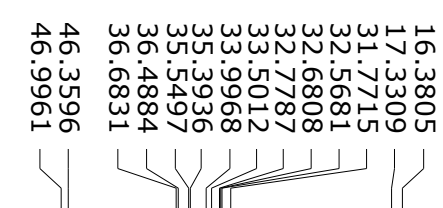 \\
\hline
\end{tabular}

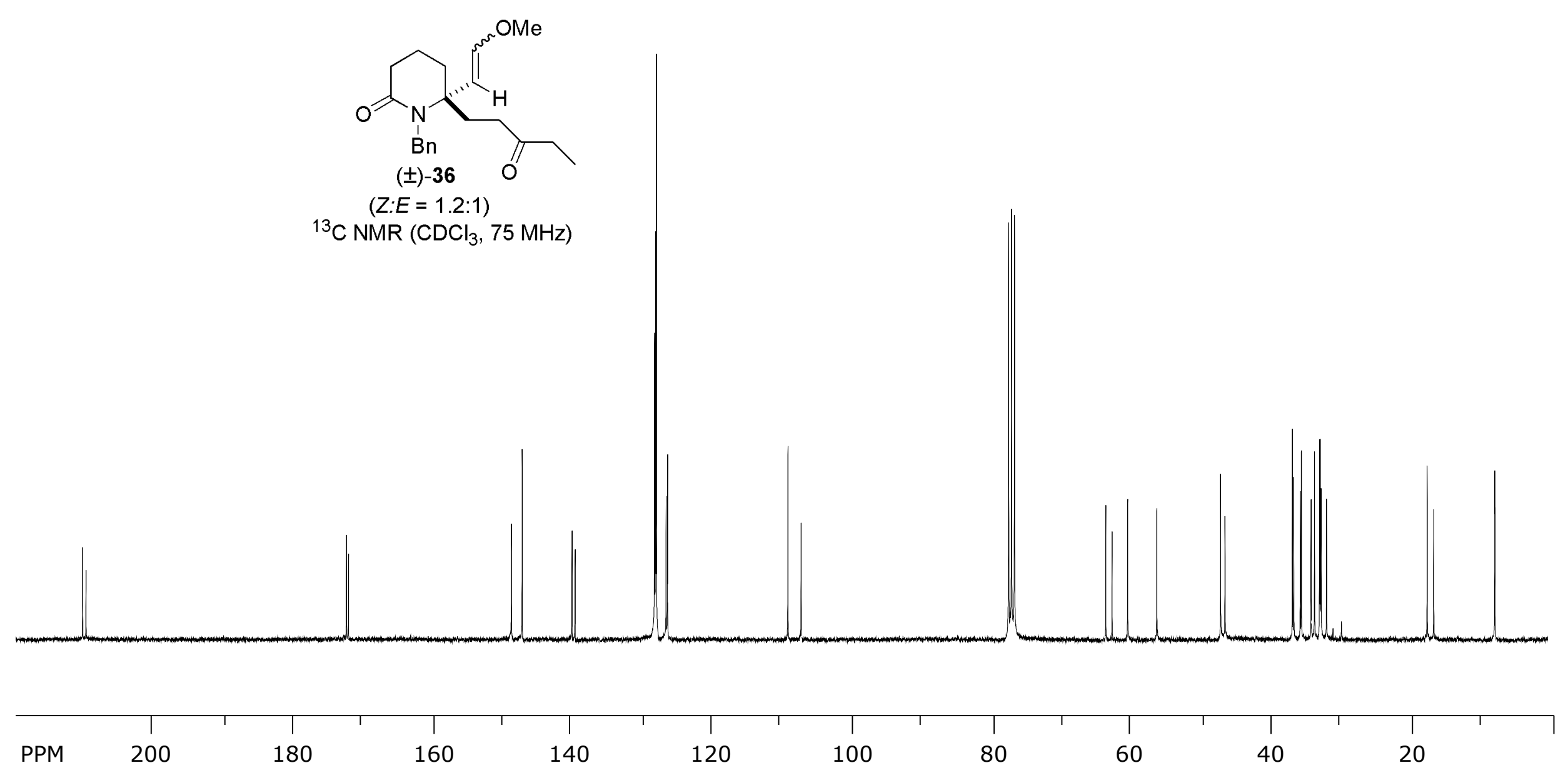




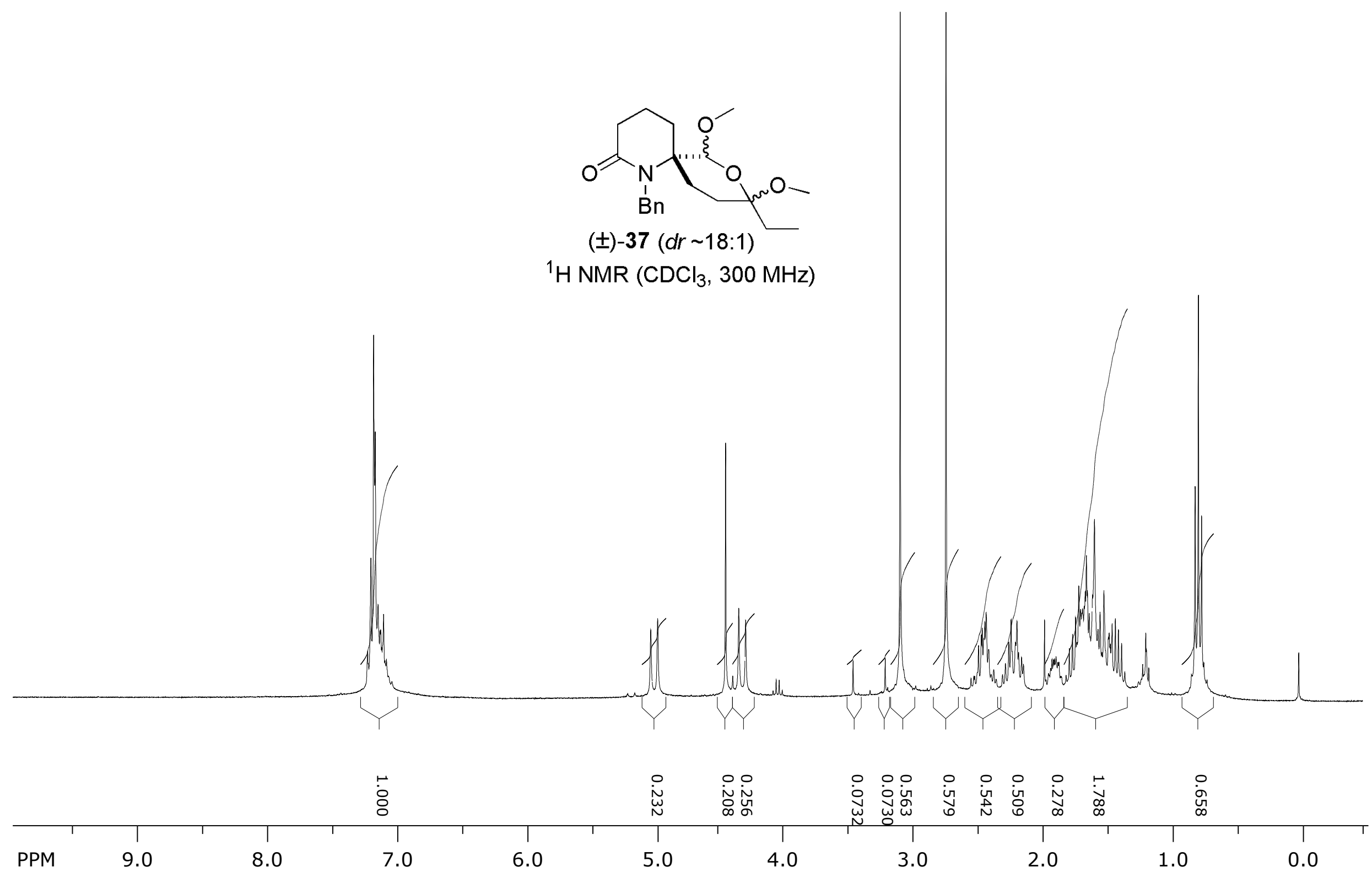




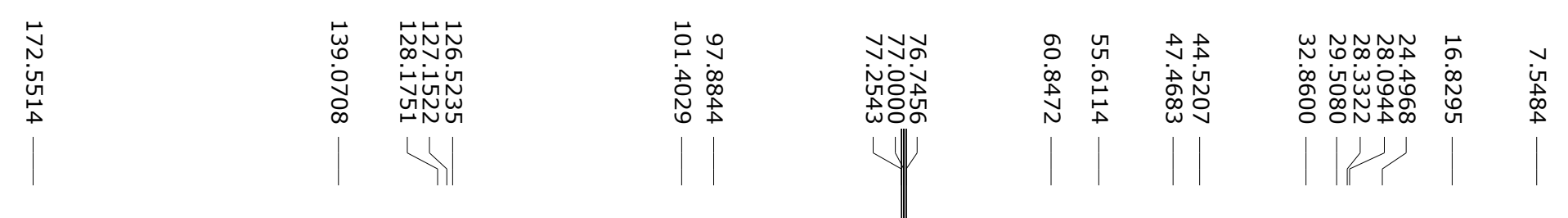

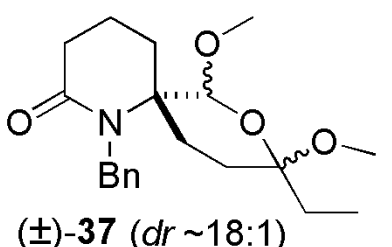

${ }^{3} \mathrm{C} \mathrm{NMR}\left(\mathrm{CDCl}_{3}, 125 \mathrm{MHz}\right)$
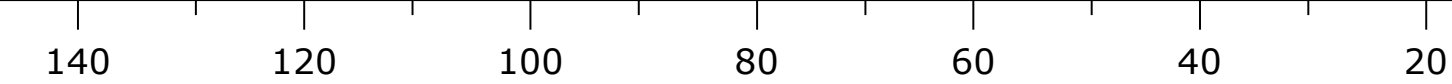


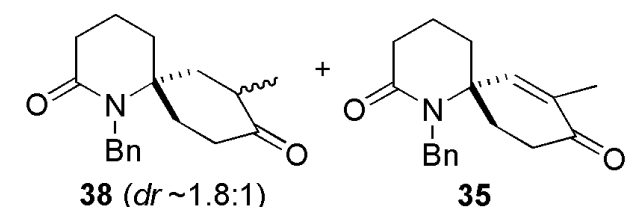

$\begin{array}{rr}38(d r \sim 1.8: 1) & 35 \\ { }^{1} \mathrm{H} \mathrm{NMR}\left(\mathrm{CDCl}_{3}, 300 \mathrm{MHz}\right)\end{array}$

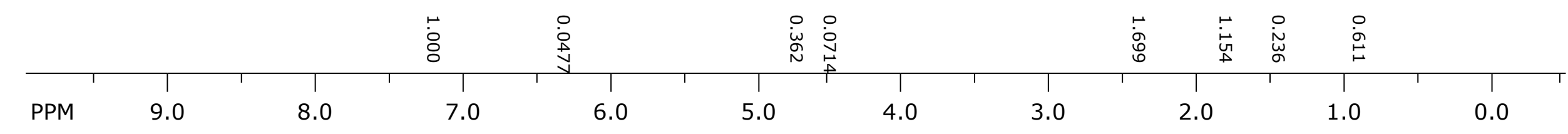




\begin{tabular}{|c|c|c|c|c|c|}
\hline 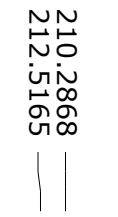 & 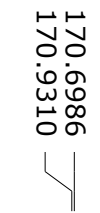 & 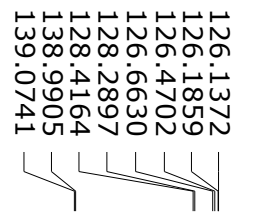 & 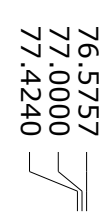 & 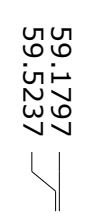 & 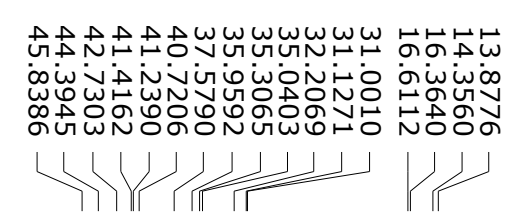 \\
\hline
\end{tabular}

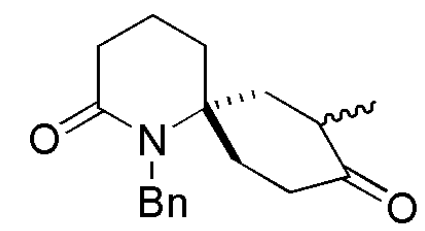

38 (dr 1:1)

${ }^{13} \mathrm{C}$ NMR $\left(\mathrm{CDCl}_{3}, 75 \mathrm{MHz}\right)$

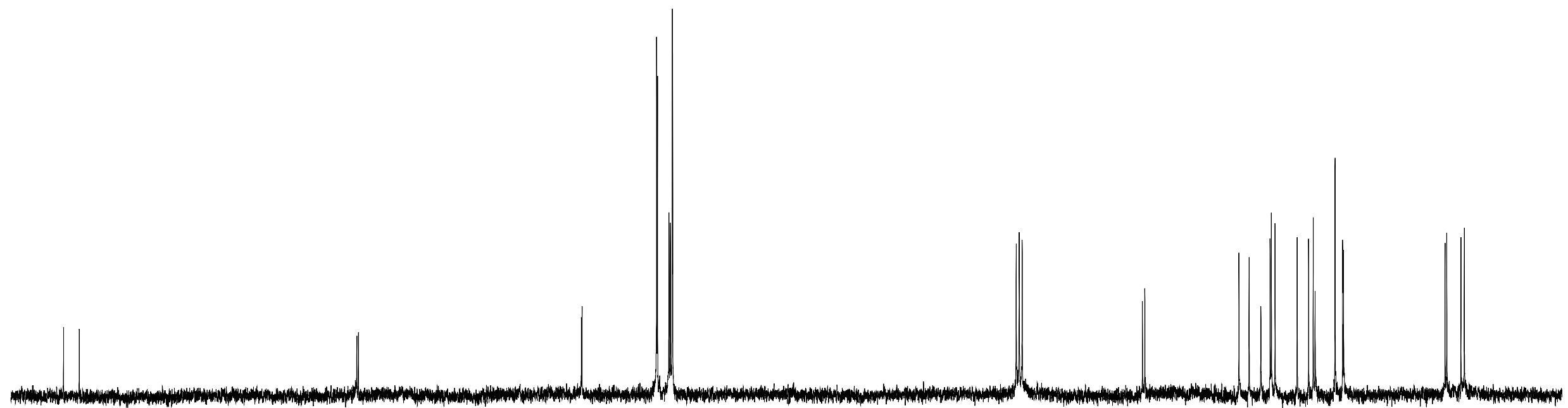




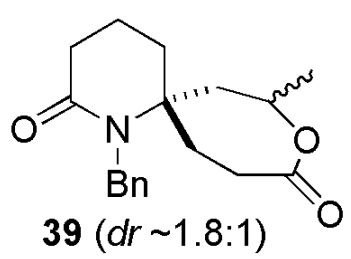

${ }^{1} \mathrm{H} \mathrm{NMR}\left(\mathrm{CDCl}_{3}, 300 \mathrm{MHz}\right)$

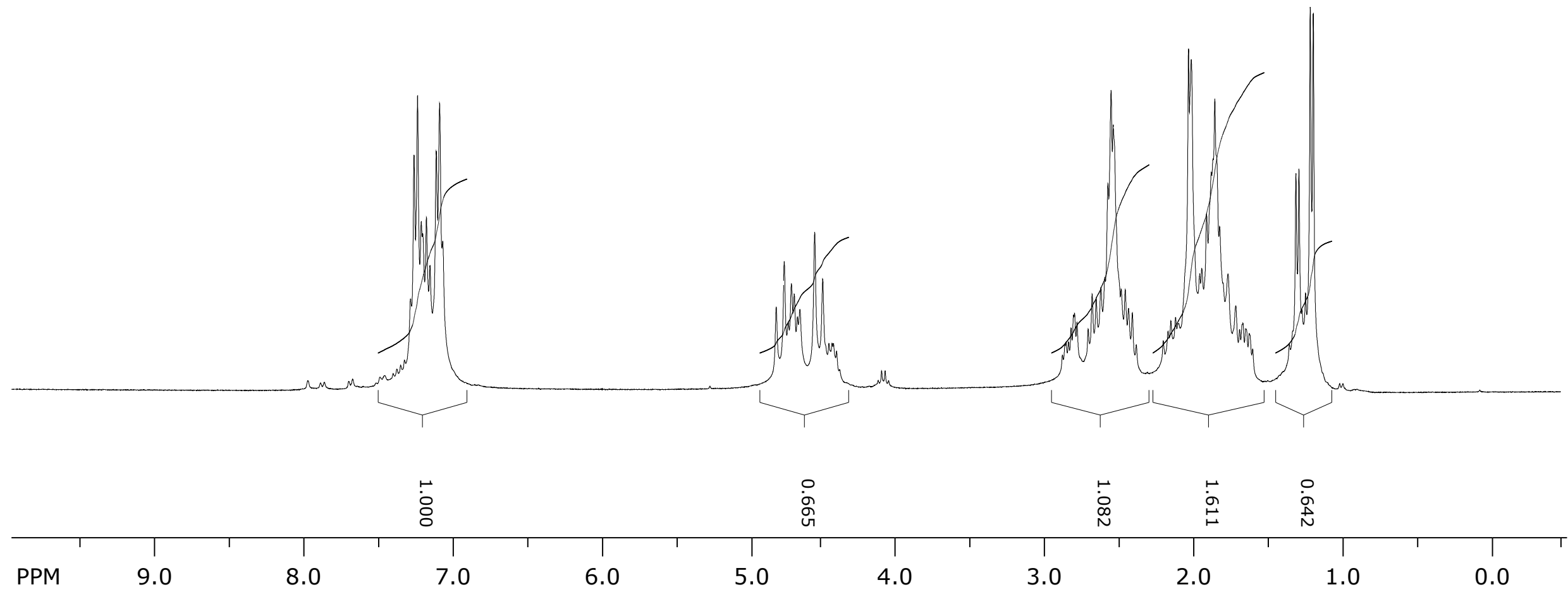




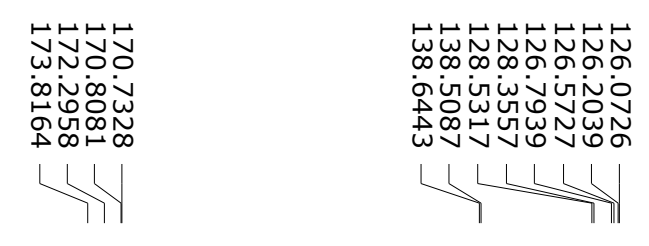

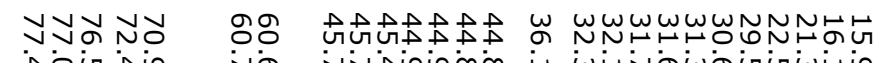

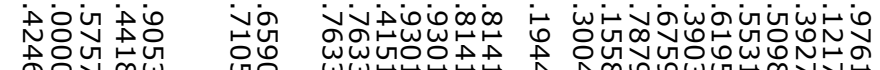
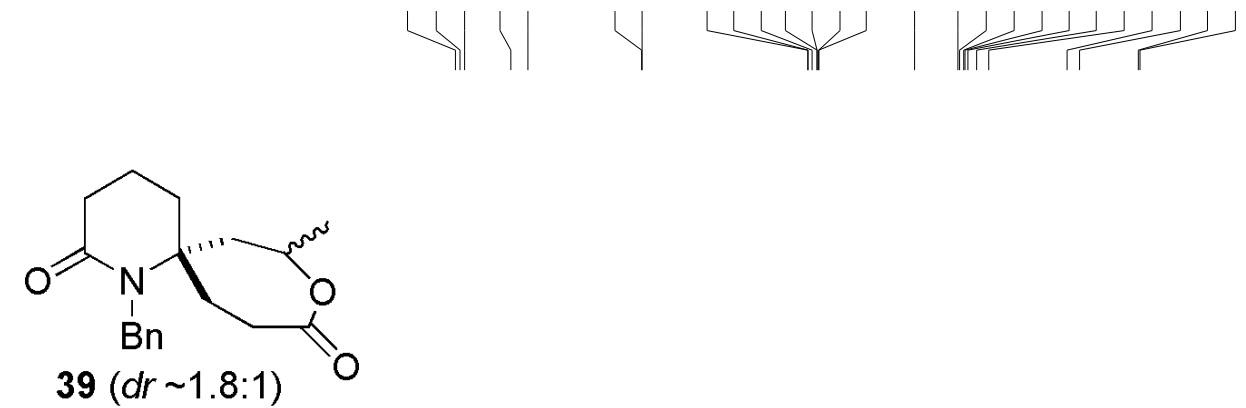

${ }^{13} \mathrm{C} \mathrm{NMR}\left(\mathrm{CDCl}_{3}, 75 \mathrm{MHz}\right)$

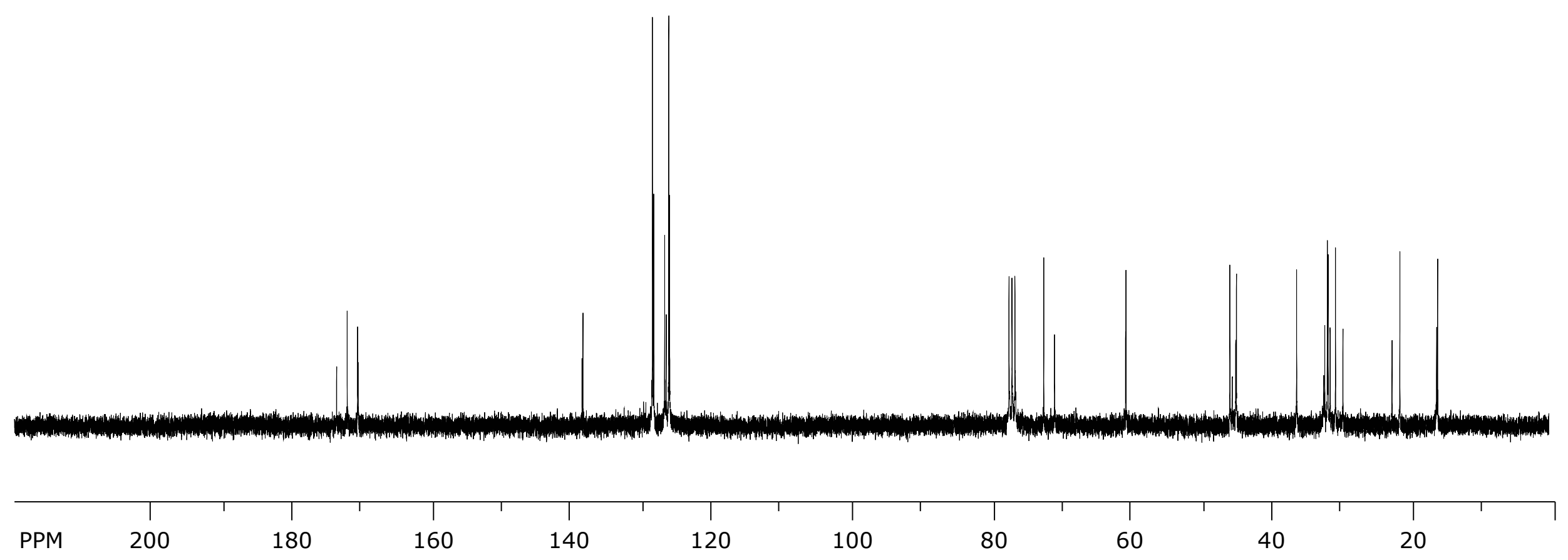




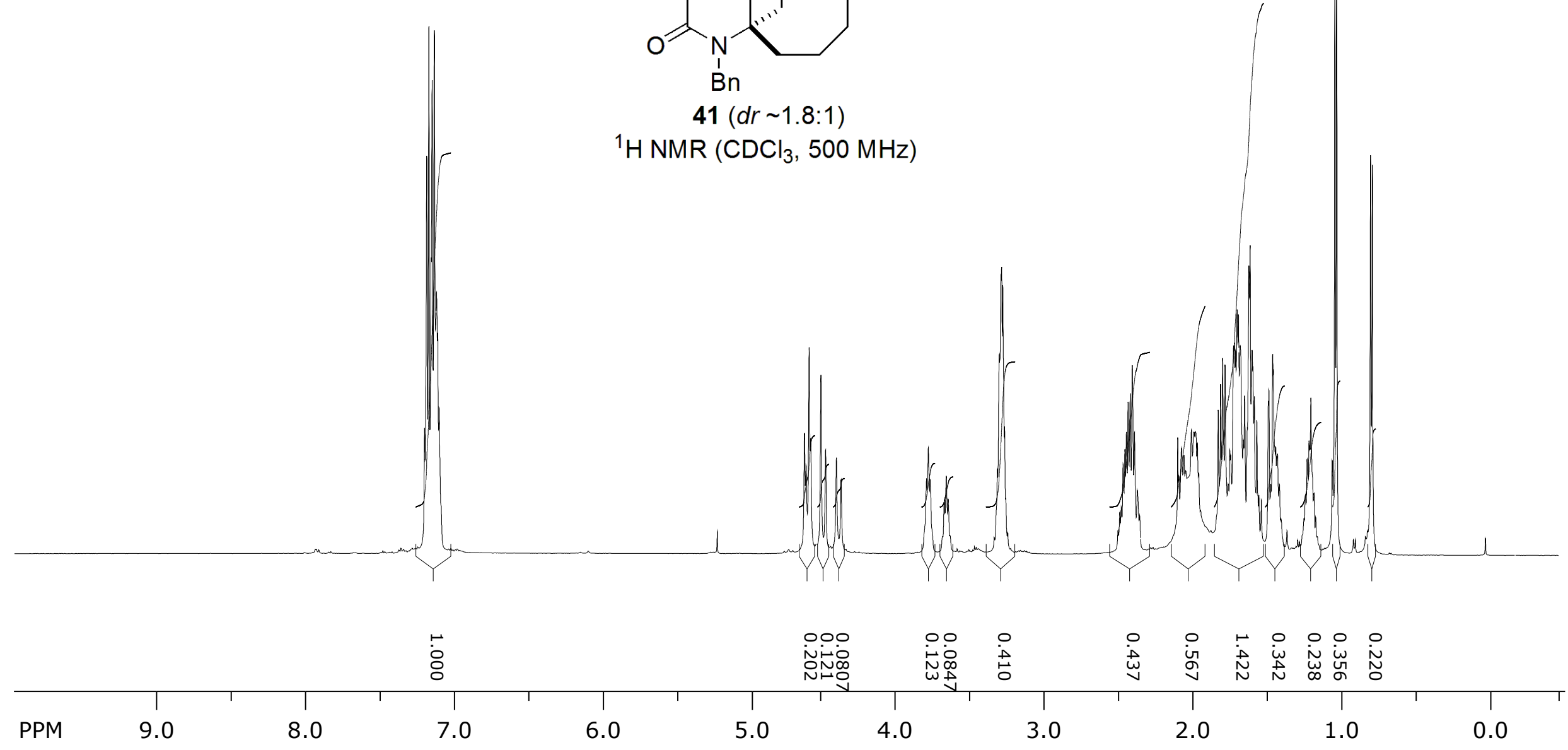



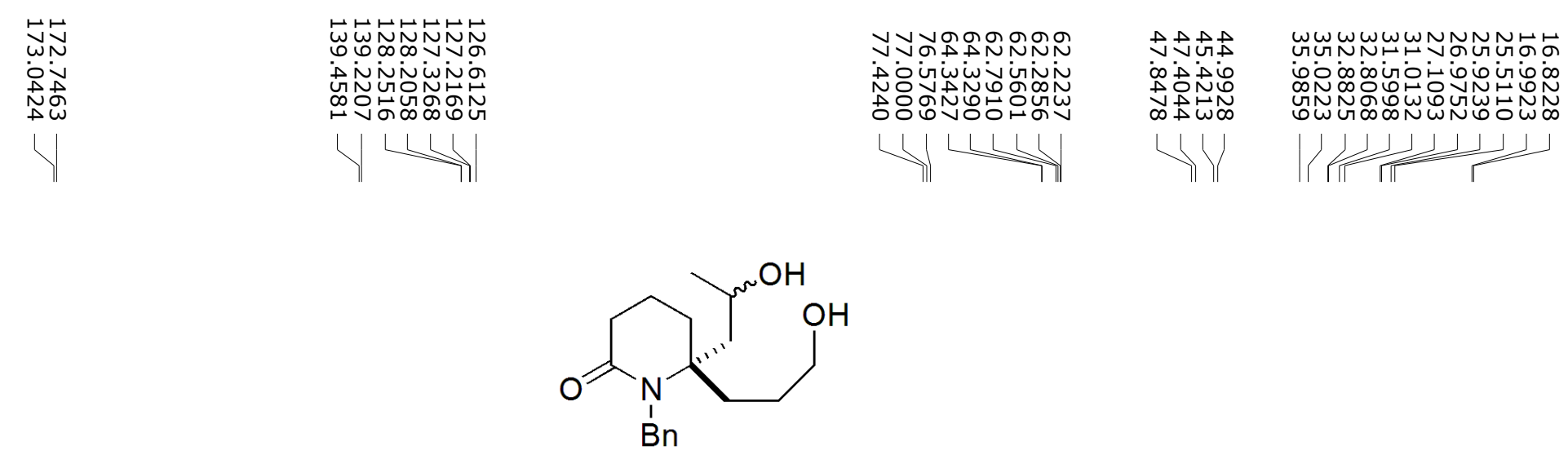

$41(d r \sim 1.8: 1)$

${ }^{13} \mathrm{C} \mathrm{NMR}\left(\mathrm{CDCl}_{3}, 75 \mathrm{MHz}\right)$

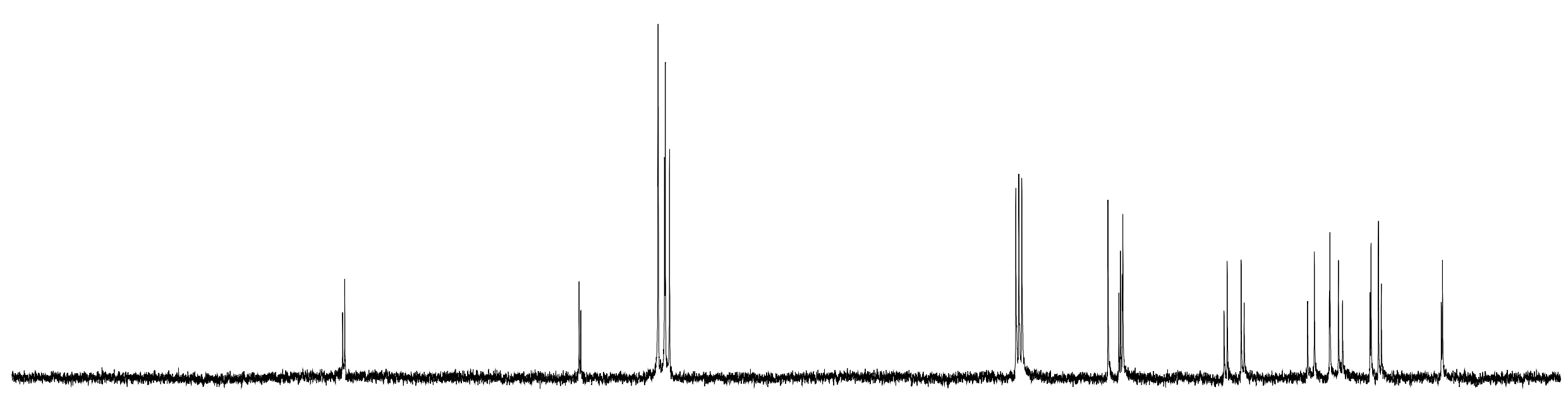




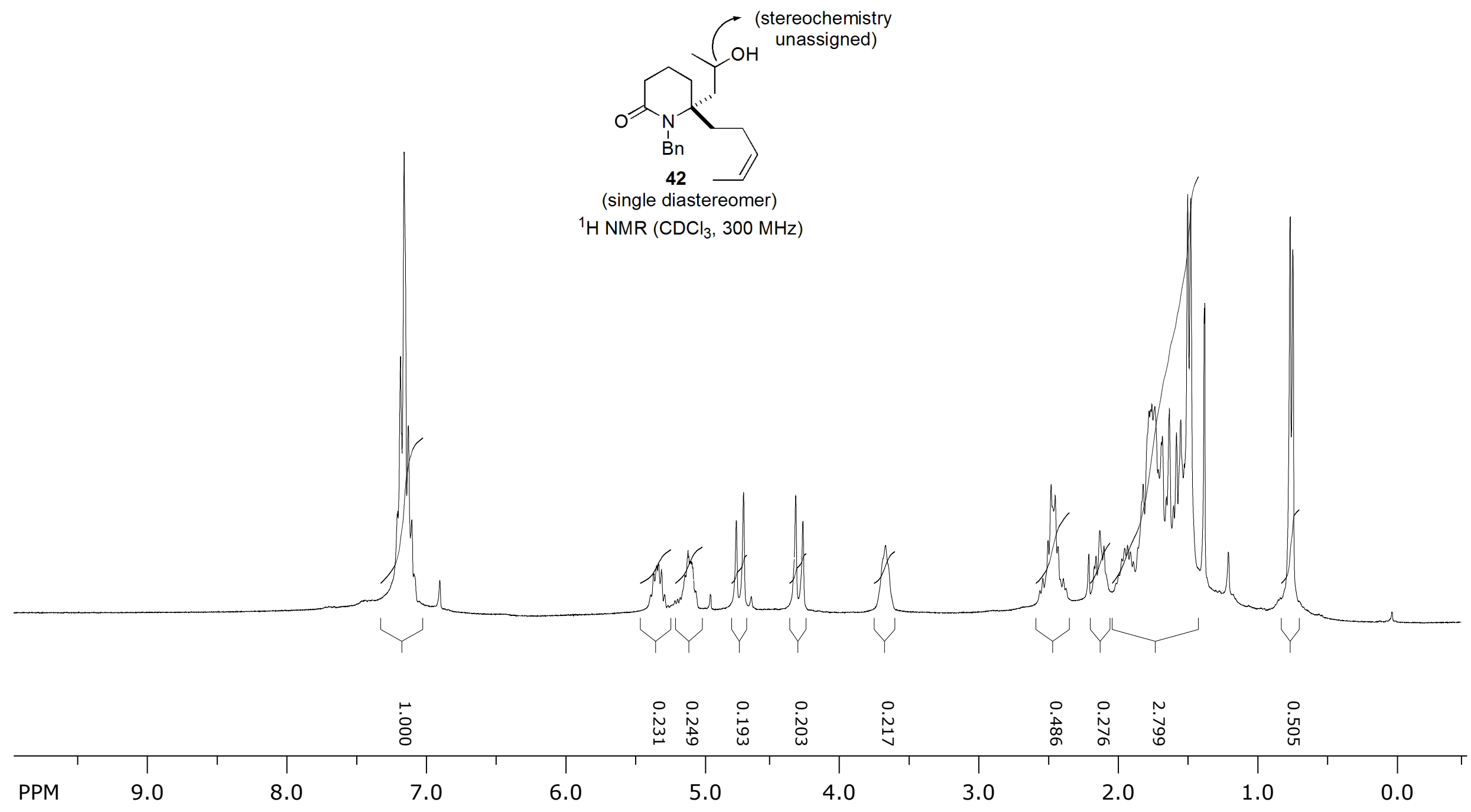



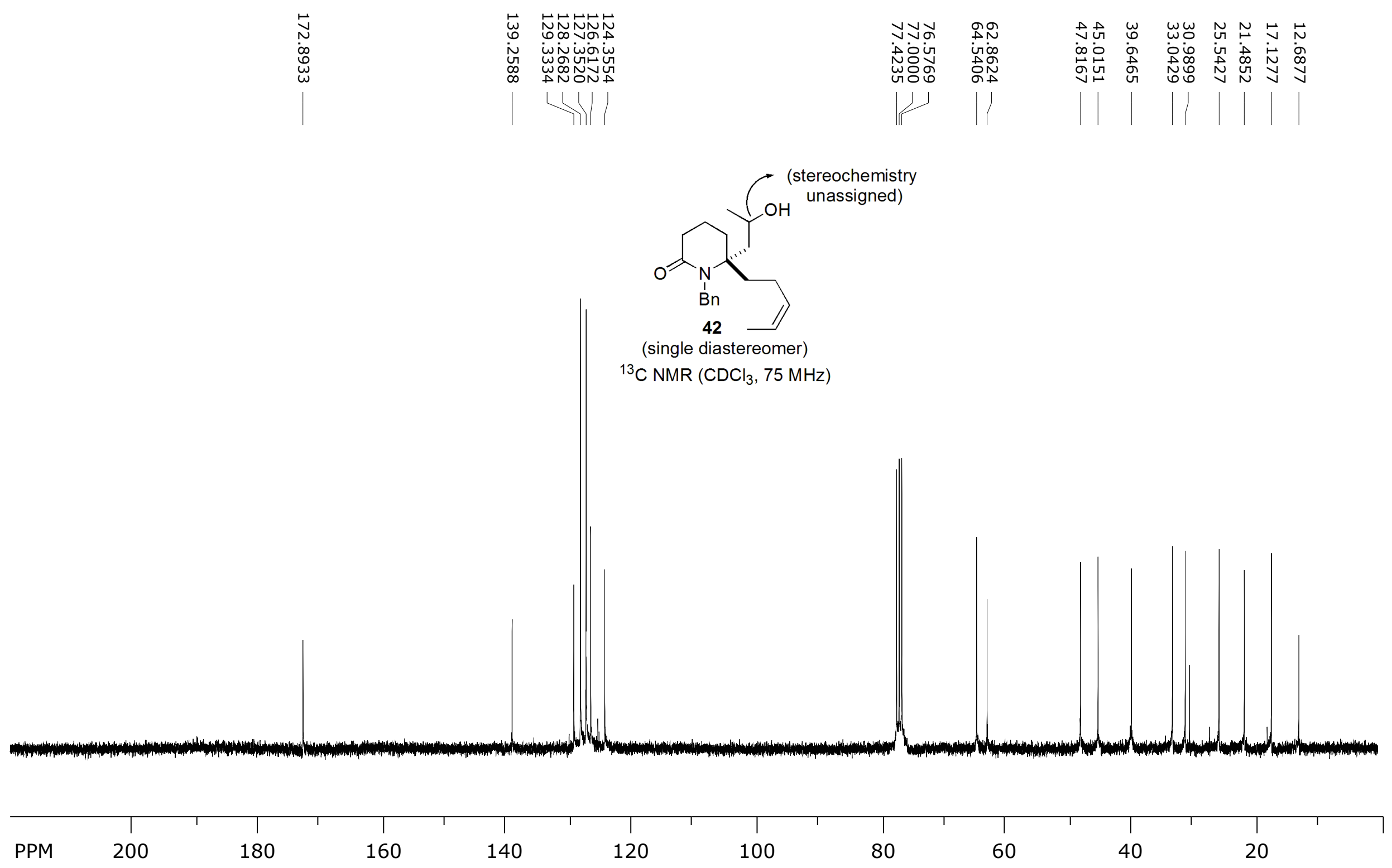


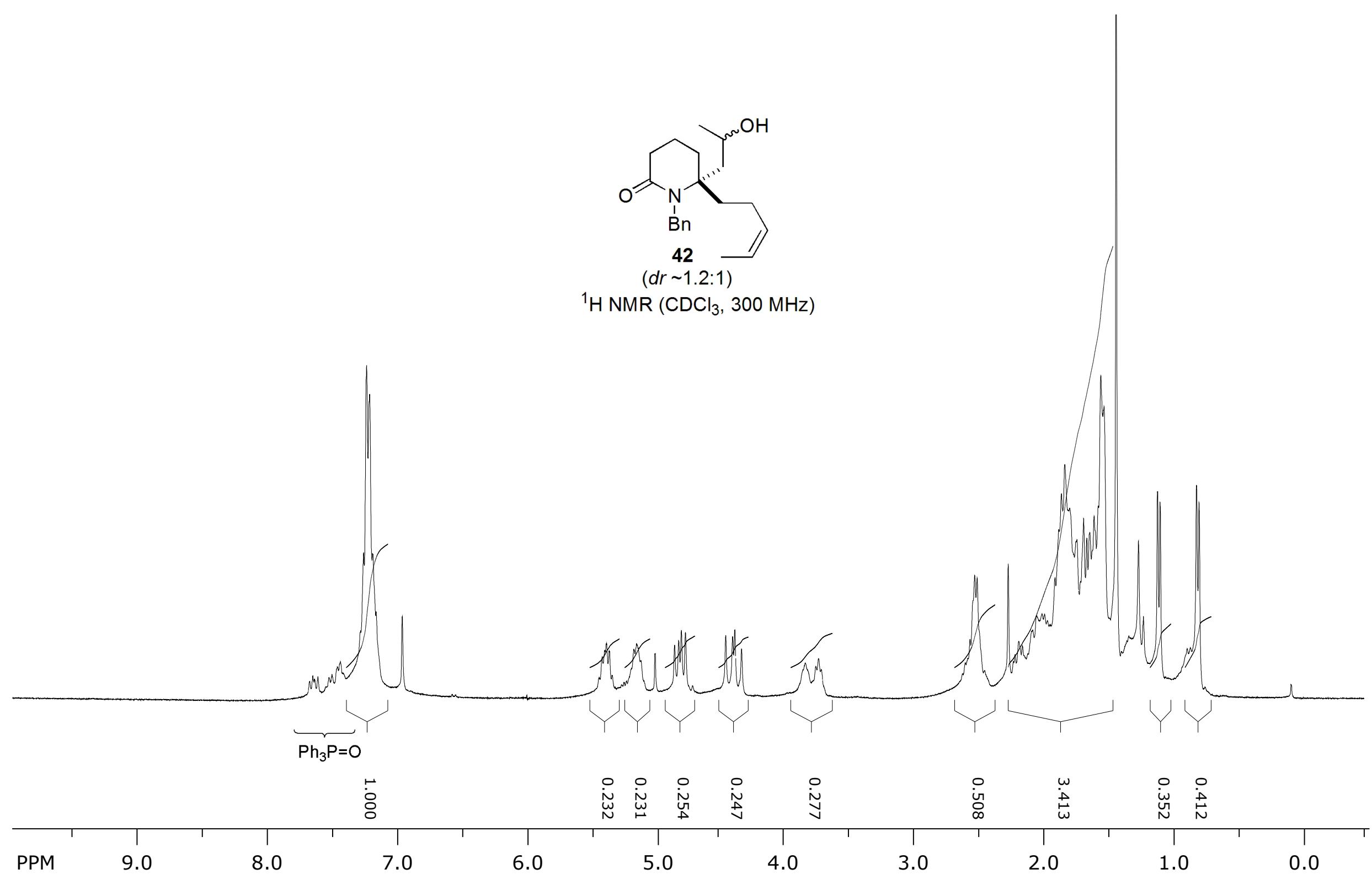




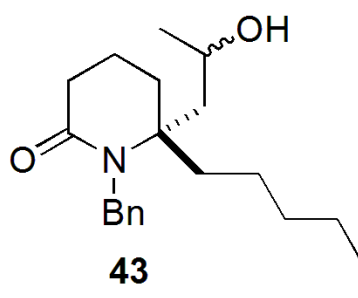

(dr $\sim 1.2: 1)$

${ }^{1} \mathrm{H}$ NMR $\left(\mathrm{CDCl}_{3}, 500 \mathrm{MHz}\right)$

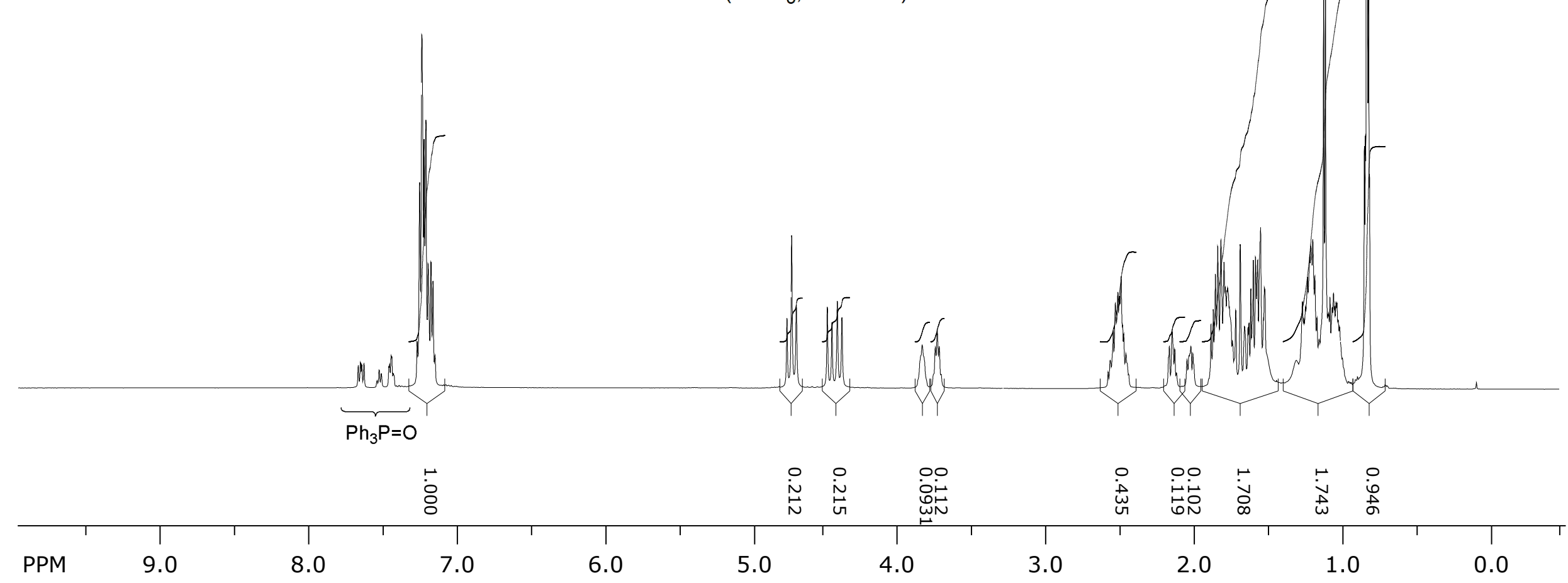




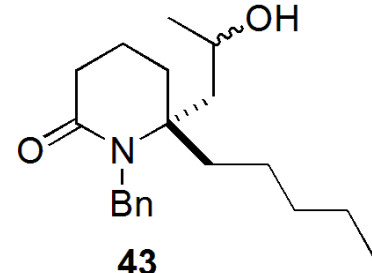

43

$(d r \sim 1.2: 1)$

${ }^{13} \mathrm{C} \mathrm{NMR}\left(\mathrm{CDCl}_{3}, 125 \mathrm{MHz}\right)$

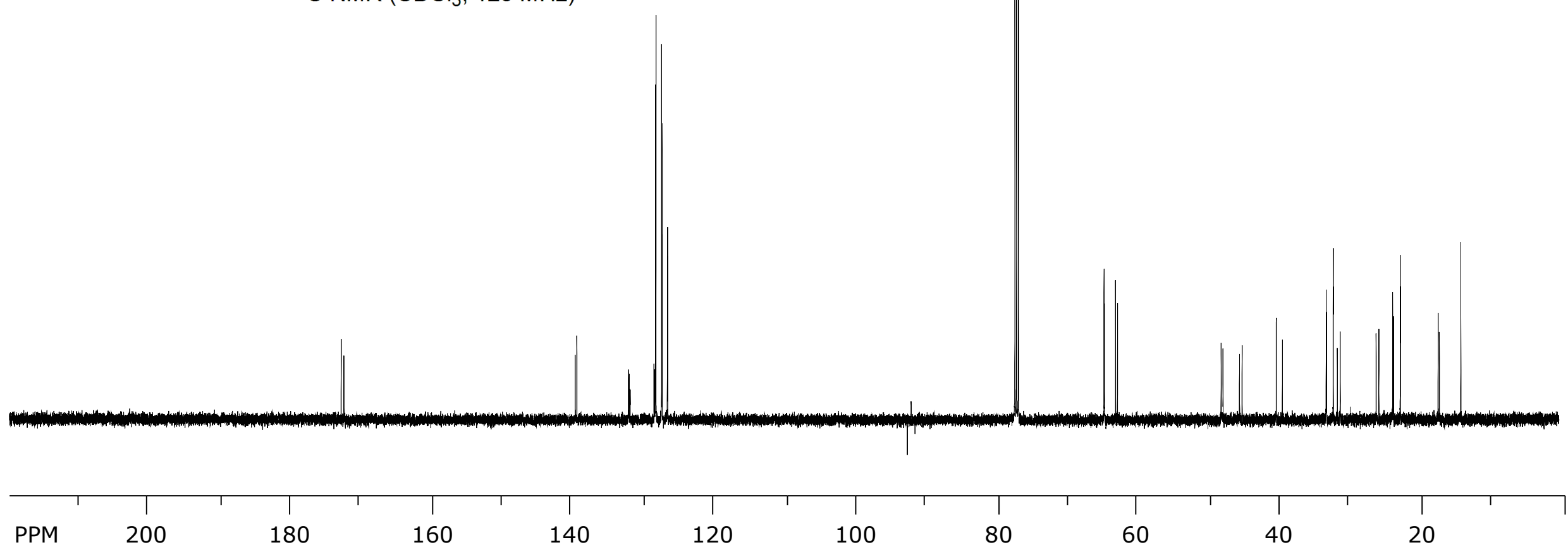




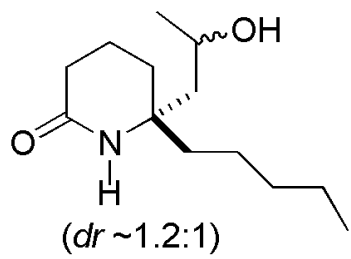

Debenzylated $\mathbf{4 3}$

${ }^{1} \mathrm{H} \mathrm{NMR}\left(\mathrm{CDCl}_{3}, 500 \mathrm{MHz}\right)$

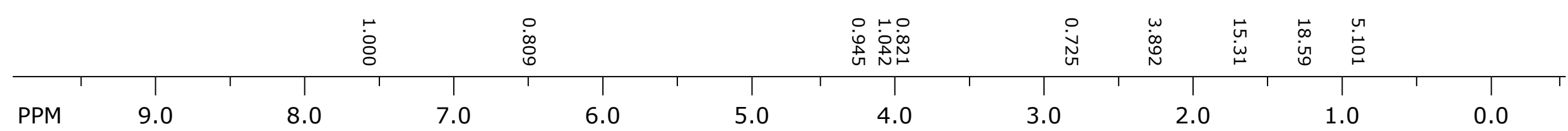




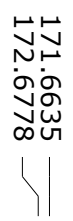

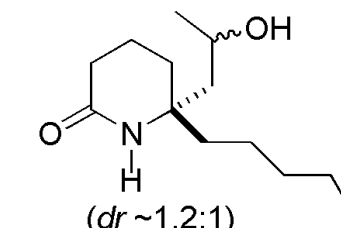

1.2:1)

Debenzylated 43

${ }^{13} \mathrm{C}$ NMR $\left({ }^{1} \mathrm{H}\right.$ coupled $)$

$\left(\mathrm{CDCl}_{3}, 125 \mathrm{MHz}\right)$

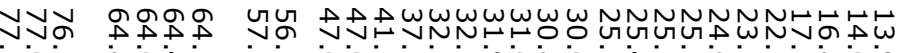

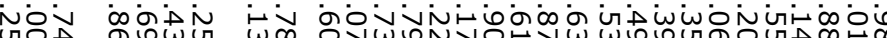

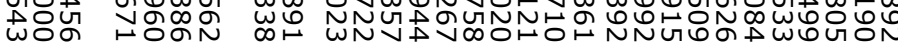

4 H

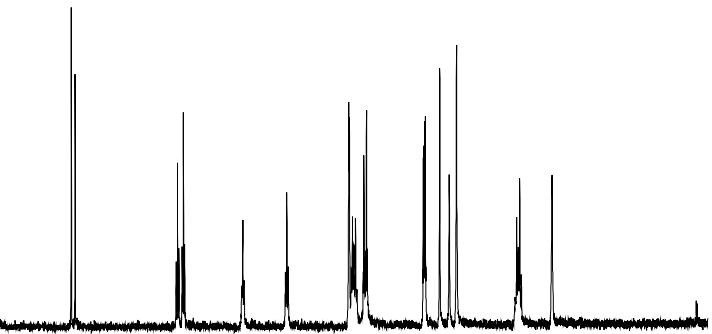




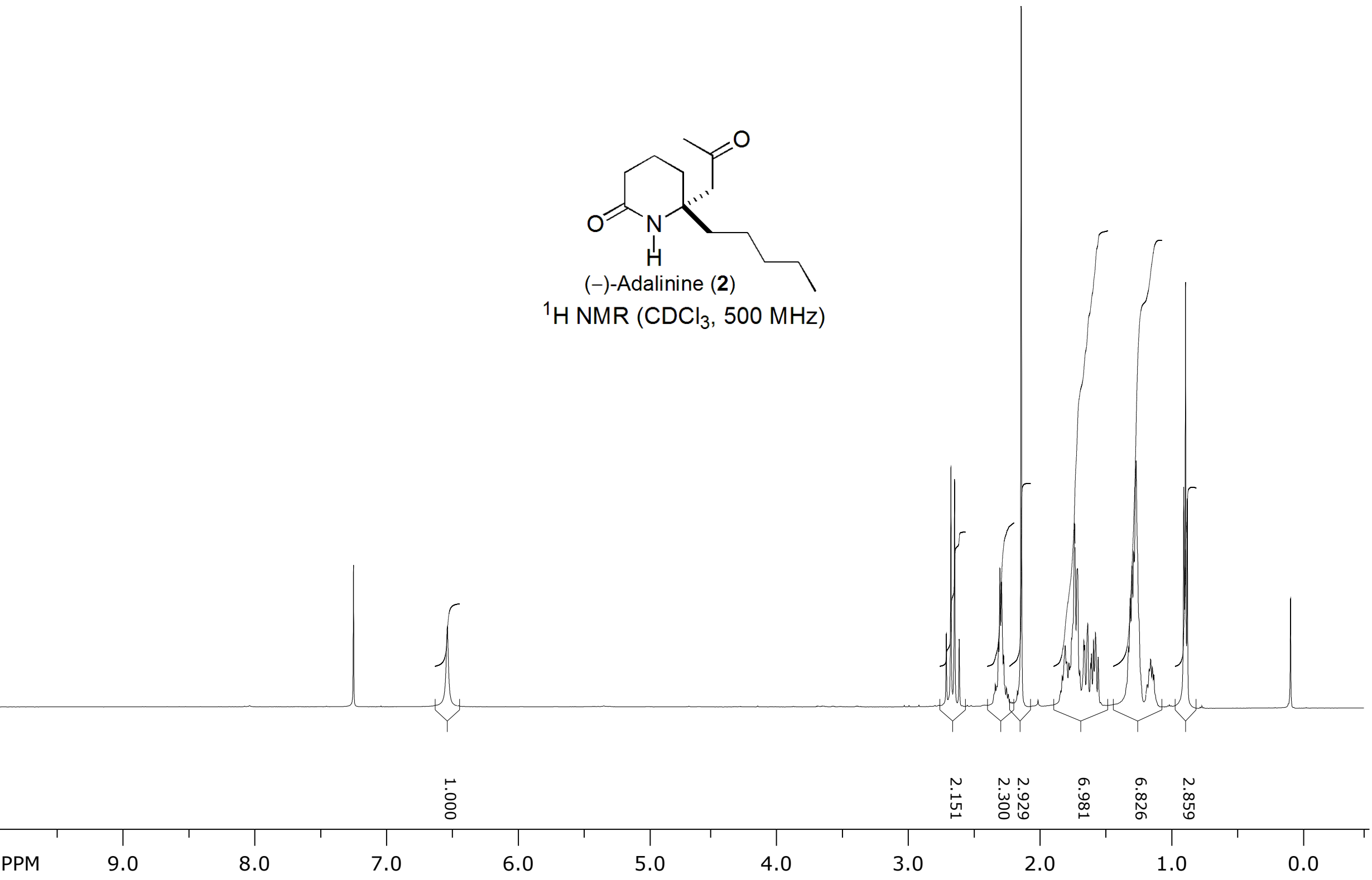




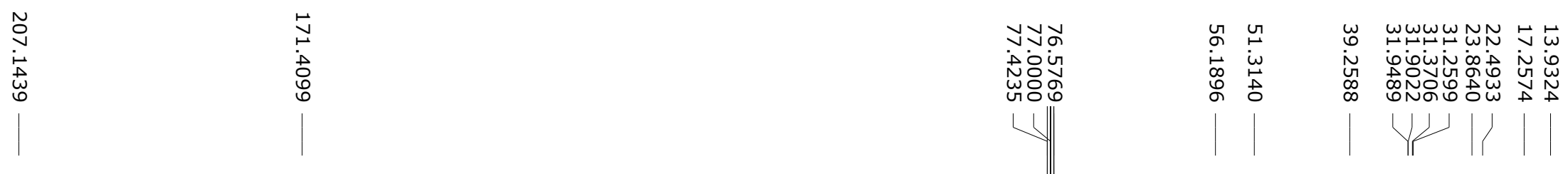

dalinine (2)

${ }^{13} \mathrm{C} \mathrm{NMR}\left(\mathrm{CDCl}_{3}, 75 \mathrm{MHz}\right)$ 


\section{$\underline{( \pm)-29}$}

HPLC: Waters 600E; Column: Chiralpak AD; 4.6 mm x 250 mm; Flow rate: $1.0 \mathrm{~mL} / \mathrm{min}$; Solvent: 95:5 v/v hexane- ${ }^{i} \mathrm{PrOH}$; Isocratic

Detector: Waters 2487; $\lambda=220 \mathrm{~nm}$

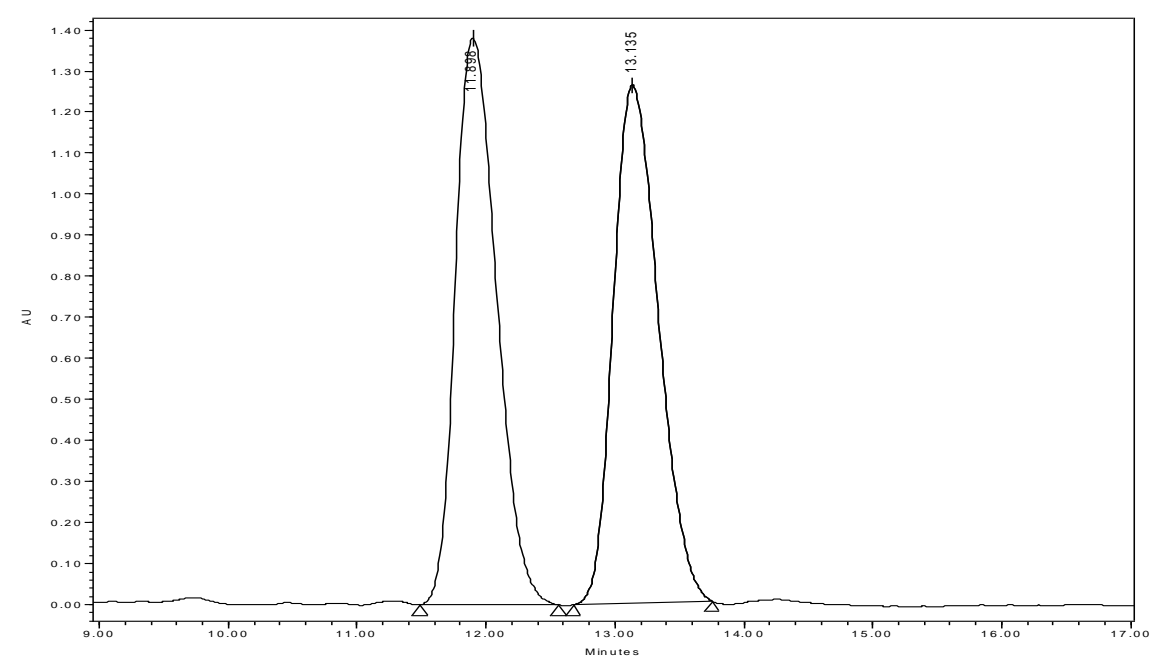

\begin{tabular}{|l|c|c|c|c|c|}
\hline & Compound & $t_{\mathrm{R}}(\mathrm{min})$ & Area & \% Area & Height \\
\hline 1 & $(R)-\mathbf{2 9}$ & 11.9 & 30666059 & 49.95 & 1379305 \\
\hline 2 & $(S)-29$ & 13.1 & 30729008 & 50.05 & 1262638 \\
\hline
\end{tabular}

(S)-29 $(98.5 \% e e)$

HPLC: Waters 600E; Column: Chiralpak AD; $4.6 \mathrm{~mm}$ x $250 \mathrm{~mm}$; Flow rate: $1.0 \mathrm{~mL} / \mathrm{min}$; Solvent: 95:5 v/v hexane- ${ }^{i} \mathrm{PrOH}$; Isocratic Detector: Waters 2487; $\lambda=220 \mathrm{~nm}$

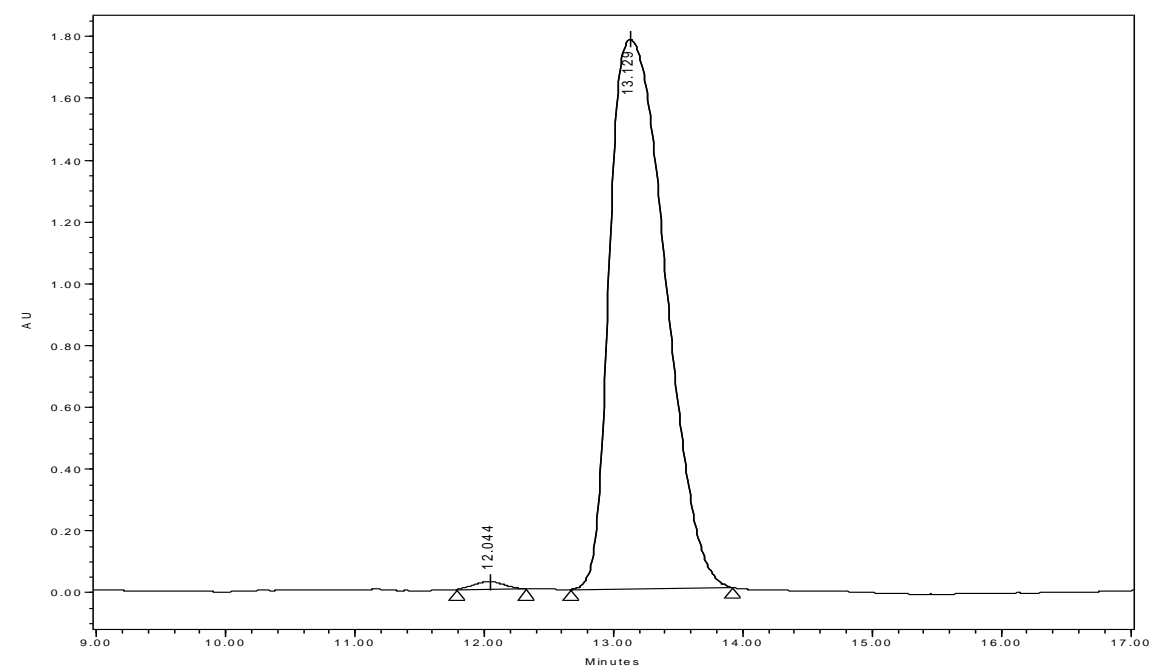

\begin{tabular}{|l|c|c|c|c|c|}
\hline & Compound & $t_{\mathrm{R}}(\mathrm{min})$ & Area & \% Area & Height \\
\hline 1 & $(R)-29$ & 12.0 & 400018 & 0.75 & 24223 \\
\hline 2 & $(S)-29$ & 13.1 & 52825898 & 99.25 & 1777293 \\
\hline
\end{tabular}




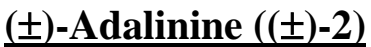

HPLC: Waters 600E; Column: Chiralcel OD; 4.6 mm x $250 \mathrm{~mm}$; Flow rate: $1.0 \mathrm{~mL} / \mathrm{min}$; Solvent: $90: 10 \mathrm{v} / \mathrm{v}$ hexane- ${ }^{i} \mathrm{PrOH}$; Isocratic

Detector: Waters $2487 ; \lambda=220 \mathrm{~nm}$

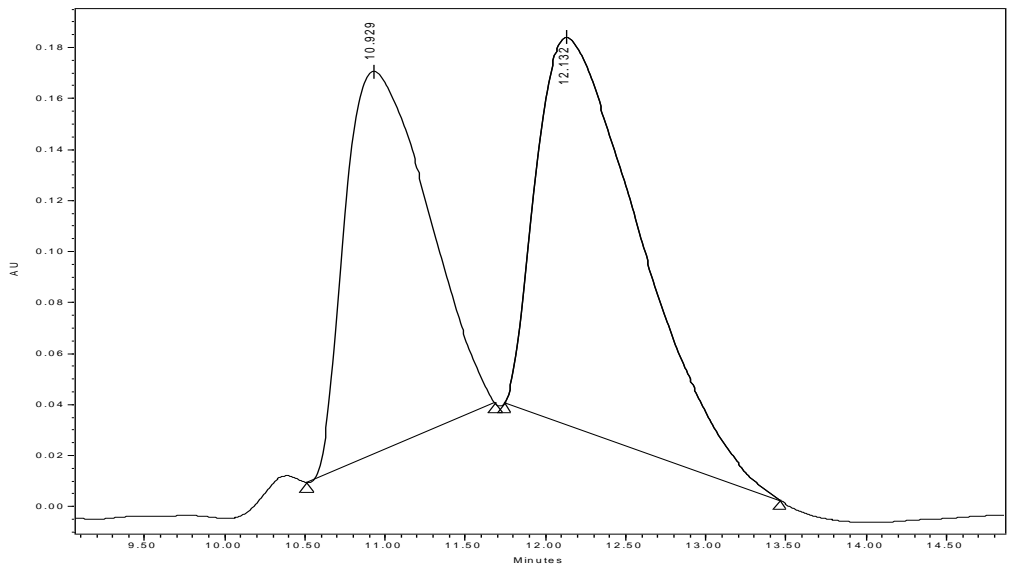

\begin{tabular}{|c|c|c|c|c|c|}
\hline & Compound & $t_{\mathrm{R}}(\mathrm{min})$ & Area & \% Area & Height \\
\hline 1 & $(R)$-Adalinine & 12.1 & 6888711 & 56.2 & 151976 \\
\hline 2 & $(S)$-Adalinine & 10.9 & 5373813 & 43.8 & 150200 \\
\hline
\end{tabular}

(R)-Adalinine $(\boldsymbol{( R ) - 2 )}(100 \%$ ee $)$

HPLC: Waters 600E; Column: Chiralcel OD; 4.6 mm x $250 \mathrm{~mm}$; Flow rate: $1.0 \mathrm{~mL} / \mathrm{min}$; Solvent: 90:10 v/v hexane- ${ }^{i} \mathrm{PrOH}$; Isocratic

Detector: Waters 2487; $\lambda=220 \mathrm{~nm}$

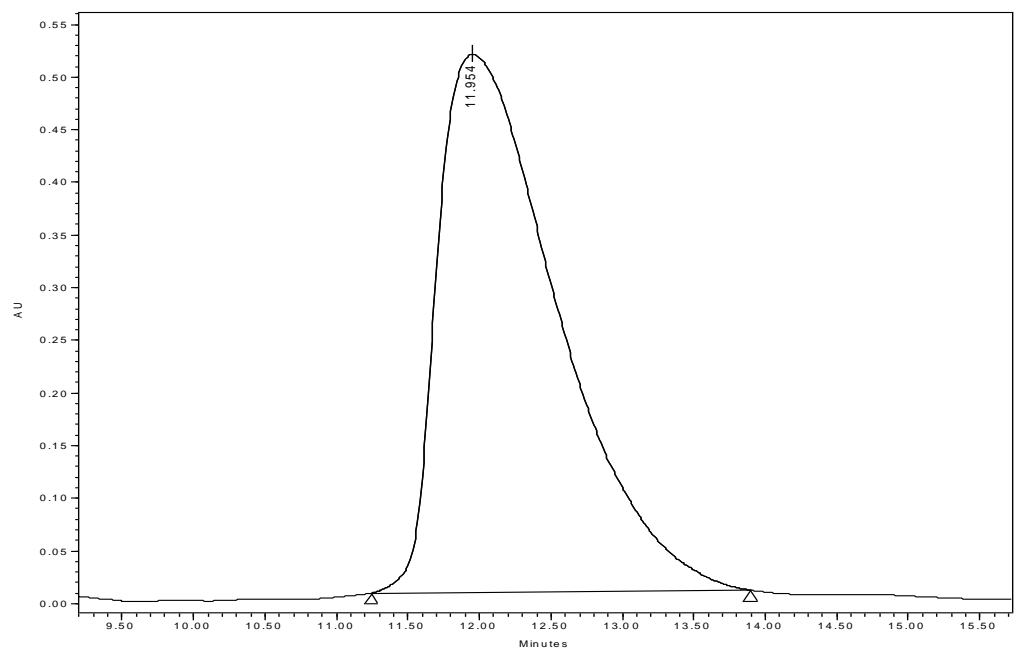

\begin{tabular}{|c|c|c|c|c|c|}
\hline & Compound & $t_{\mathrm{R}}(\mathrm{min})$ & Area & \% Area & Height \\
\hline 1 & $(R)$-Adalinine & 11.9 & 29792607 & 100.00 & 511399 \\
\hline
\end{tabular}

